\title{
RESPUESTA SÍSMICA LOCAL EN PUNTOS DE LA CIUDAD DE GRANADA
}

MÁSTER UNIVERSITARIO DE ESTRUCTURAS

UNIVERSIDAD DE GRANADA

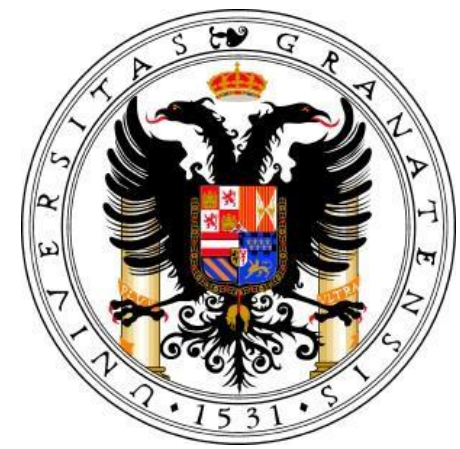

Autor:

Francisco Javier Morales Gámiz

Tutores:

Gerardo Alguacil y Francisco Vidal 


\section{ÍNDICE}

\section{1.- Introducción}

1.1.- Interés

1.2.-Planteamiento y objetivos

\section{2.- Geología de la zona en estudio}

2.1.- Encuadre geológico regional

2.2.- Encuadre geológico local

\section{3.- Adquisición y tratamiento de datos de partida}

3.1.- Método SPAC

3.1.1.- Fundamento teórico

3.2.- Aplicación del SPAC en diferentes puntos de Granada

3.2.1.- Aplicación al caso en estudio

3.2.2.- Puntos de estudio

3.2.3.- Medidas de ruido obtenidas

3.2.4- Resultados obtenidos

\section{4.- Amplificación sísmica CAMBIAR A CAPITULO 2}

4.1.- Introducción

4.2.- Efecto de sitio

4.3.- Respuesta sísmica de los suelos

4.3.1.- Propiedades dinámicas de los suelos

4.3.2.- Efectos del suelo en superficie

\section{5.- Metodología para el cálculo del efecto de sitio}

5.1.- Método de Nakamura

5.1.1.- Fundamento teórico 
5.1.2. Ventajas y limitaciones del método

5.2.- Métodología de Tsai

5.1.1.- Fundamento teórico

\section{6.- Cálculo y resultados}

6.1.- Cálculo del efecto de sitio

6.1.1.- Método de Nakamura

6.1.2.- Métodología de Tsai

6.2.- Comparativa de resultados

6.3.- Comparativa de resultados con datos de otro SPAC de mayor amplitud.

\section{7.- Aplicación a un terremoto tipo}

7.1.- Introducción

7.2.- Terremoto de "Valnerina" (Italia)

7.2.1.- Características

7.2.2.- Resultados

7.3.- Terremoto de "Basso Tirreno" (Italia)

7.3.1.- Características

7.3.2.- Resultados

8.- Discusión de resultados

9.- Conclusiones

10.- Referencias

APÉNDICE I. Implementación en MatLab de la metodología de Tsai 


\section{Agradecimientos}

Quiero dar las gracias a los profesores Gerardo Alguacil de la Blanca y Francisco Vidal y por extensión a todo el Instituto Andaluz de Geofísica y al departamento de Física Teórica y del Cosmos de la Universidad de Granada, sin los cuáles hubiera sido imposible realizar todo el trabajo.

Junto con ellos al profesor Manuel Navarro de la Universidad de Almería por haberme facilitado los datos del SPAC y gran cantidad de información.

También recordar a la Base Europea de Terremotos y al Instituto Geológico Nacional por los datos facilitados.

Este trabajo ha sido parcialmente realizado dentro del marco del proyecto CGL2011-30187c02-01-02. 


\section{INTRODUCCIÓN Y OBJETIVOS}

\section{1.- Interés del trabajo}

La experiencia a lo largo de los años nos ha llevado a determinar que la influencia de las condiciones geológicas y topográficas durante el desarrollo de un terremoto son notables produciéndose una modificación de la señal sísmica. Esta modificación se conoce con el nombre de efecto local.

Dicha alteración consiste en la amplificación o en la reducción de la señal, así como una mayor o menor duración de la misma y una modificación de su contenido frecuencial, dando lugar a unas características de la señal íntimamente ligadas con las condiciones locales.

El análisis de las anomalías de la intensidad sísmica obtenidas a partir de datos macrosísmicos permitió establecer la evidencia que los terrenos blandos amplifican los efectos de los terremotos.

Se han desarrollado un gran número de escalas macrosísmicas que recogen y clasifican en grados los efectos que provoca el terremoto sobre las edificaciones, las personas y el medio natural. Las escalas más importantes actualmente en uso son: la escala MSK (Medvedev, Sponhauer, Kärnik, 1963) (que era la escala macrosísmica europea anterior a la EMS actual), la escala MM (de Mercalli Modificada), la escala JMA (Japanese Metereological Agency), la escala EMS98 (European Macroseismic Scale, 1998) y la escala China que equivale a la MM.

Las anomalías de la intensidad sísmica analizadas han permitido realizar correlaciones entre la intensidad y las condiciones geológicas y topográficas específicas de la zona (sobre todo de las primeras) con el objetivo de poder realizar mapas de zonificación y microzonnificación sísmicas que permiten delimitar distintas zonas en función de su diferente capacidad para amplificar el movimiento del terreno y, consecuentemente, el daño sísmico. Sin embargo, la limitación de los estudios de zonación y microzonación sísmicas radica principalmente en que la intensidad es un parámetro obtenido a partir de los efectos producidos por la sacudida sísmica con el que se obtiene un valor para estimar la propia acción del movimiento del suelo; por lo tanto, no expresan adecuadamente otro tipo de características, como los periodos dominantes de la vibración, el desigual contenido energético en esos períodos, la interacción suelo-estructura, lod fenómenos de resonancia que se producen cuando las frecuencias de vibración del suelo y de la estructura son próximas, etc. Estos fenómenos dan lugar a daños muy diferentes a los esperados en una zona ya que en cada subzona, con condiciones de sitio diferentes, habrá una respuesta del terreno también diferente.

Las roturas de taludes, caídas de rocas, o fenómenos de licuefacción y asentamientos del suelo durante o inmediatamente después de la sacudida sísmica son otros efectos inducidos que deben considerarse porque también provocan mayores daños potenciales en las estructuras. 
Por lo tanto, la identificación de las frecuencias predominantes del suelo y la cuantificación de la amplificación de la señal sísmica constituyen uno de los principales intereses en ingeniería sísmica.

Para conseguir estas metas se recurre a métodos empíricos, como p.e. la técnica de Nakamura (1989) y a la simulación numérica de los efectos sísmicos locales como p.e. el método de Tsai (1969), que son los que desarrollaremos en este trabajo.

\section{2.-Planteamiento y objetivos}

El planteamiento del presente estudio es sencillo al tratarse de un problema directo.

Partiremos de las medidas de ruido ambiental en diferentes puntos de la ciudad de Granada. Posteriormente estos datos han sido tratados por la técnica SPAC obteniéndose unos modelos de velocidades de ondas $\mathrm{S}$. Estos nos permitirán tener una idea aproximada del perfil geológico en los puntos en estudio, es decir de su estructura superficial en términos de velocidades de ondas de cizalla, Vs.

Con estos datos, pasaremos a aplicar los métodos de Nakamura (1989) y Tsai (1969) para el cálculo de la amplificación en terrenos, basándonos en unas estructuras geológicas ya obtenidas, en diferentes lugares de la ciudad, objeto del presente trabajo. Para ello se utilizarán aplicaciones realizadas en código MatLab. Para el método de Tsai se utiliza un código realizado especialmente para el presente trabajo y basado en los trabajos de Herak (2007) mientras que para el de Nakamura se utilizó ya lo implementado anteriormente.

Finalmente se compararán los resultados obtenidos con ambos métodos y se aplicará a un par de terremotos tipo a los posibles que pueden acontecer en el entorno en estudio con objeto de ver como se alteran los parámetros de dichos terremotos.

Por tanto, el objetivo general de este estudio es aplicar la metodología desarrollada por Tsai (1969) para obtener un modelo de amplificación del suelo y aplicarlo a los 13 puntos de la ciudad de Granada donde se conoce su estructura superficial.

Esto se concreta en los siguientes objetivos específicos:

- Implementar una metodología para el método de Tsai (1969) que permita facilmente obtener una función de amplificación

- Obtener los resultados de amplificación para los puntos en estudio

- Zonificación de la ciudad de Granada en función de los valores de amplificación calculados 


\section{2.- ENCUADRE GEOLÓGICO}

\section{1.- Encuadre geológico regional}

La zona objeto de estudio se encuentra enclavada dentro de las Cordilleras Béticas, las cuáles forman, junto con las Cordilleras del Rif del norte de África, el segmento más occidental del orógeno alpino mediterráneo. Estas dos cordilleras separadas en la actualidad por la cuenca neógena de Alborán, se localizan entre dos zócalos hercínicos, el ibérico al norte y el africano al sur, de acuerdo con lo reproducido en la figura 2.1.1

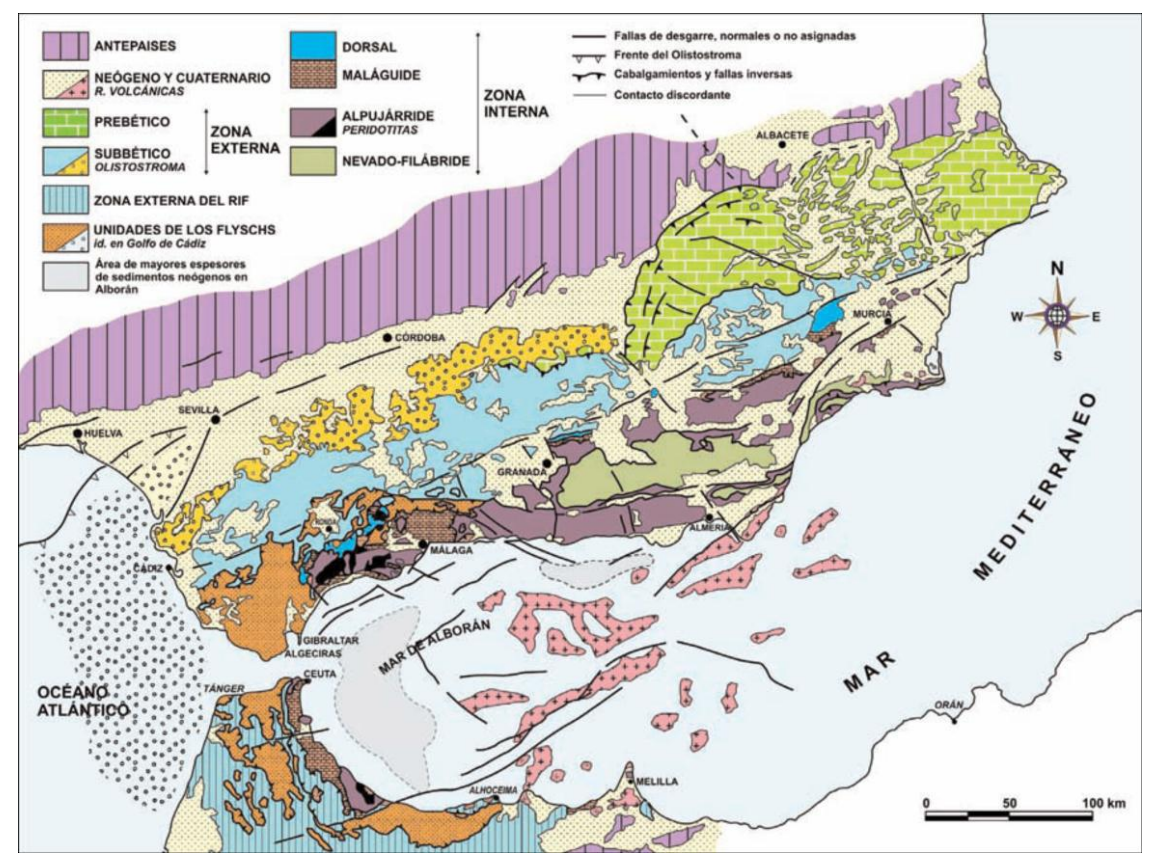

Figura 2.1 Mapa geológico regional simplificado (Sanz de Gadeano et al., 2007)

Las cordilleras Béticas se formaron como consecuencia del régimen compresivo, que comenzó a finales del Cretácico, y en ellas se pueden distinguir distintos dominios o zonas siendo las más importantes, ordenadas de norte a sur, las zonas externas y las zonas internas (también denominadas Dominio de Alborán). Estas zonas, separadas y diferenciadas por un contacto tectónico, presentan además un origen paleogeográfico distinto. 
Además de estas dos grandes zonas, existen otros dominios entre los que destacan las depresiones post-orogénicas terciarias, rellenas de materiales terciarios y cuaternarios procedentes de la erosión de los relieves circundantes.

La zona objeto de estudio se encuentra dentro de la Depresión de Granada (Figura 2.2) , una de estas cuencas post-orogénicas que se sitúa de manera discordante sobre el contacto entre las zonas externas e internas. La historia geológica de la depresión de Granada comienza a mediados del Mioceno, cuando se reanuda la sedimentación tras un periodo marcado por importantes inestabilidades tectónicas (Cemosa, 2005).

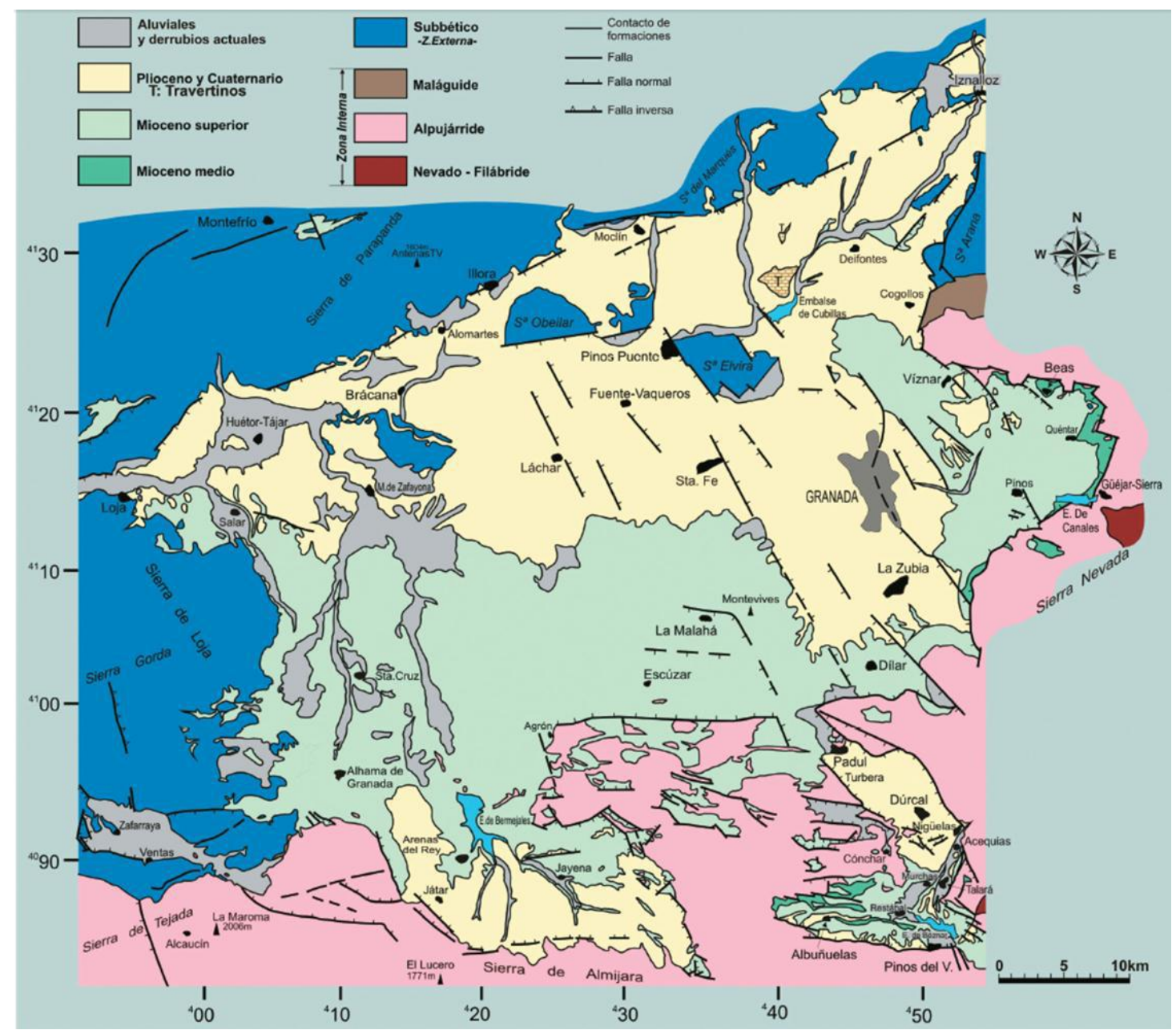

Figura 2.2: Esquema Geológico de la cuenca de Granada (Sanz de Galdeano, et al. 2001)

En el Mioceno superior se produce una transgresión marina, así como una elevación de relieves, que originan una importante avalancha conglomerática que se adosa a los bordes de la cuenca. En el Tortoniense superior esta cuenca marina se continentaliza y es sustituida por una cuenca endorreica de sedimentación lacustre.

En el Mioceno terminal debió ocurrir un rejuvenecimiento del relieve y un cambio climático importante que condicionó la instauración de un régimen fluvio-lacustre con importantes aparatos fluviales provenientes de las sierras circundantes. En el Pleistoceno medio-superior se reactivaron y crearon fracturas de importante salto que fueron y siguen siendo en la actualidad rellenadas por los aportes del río Genil y sus afluentes. 


\section{2.- Encuadre geológico local}

De cara al encuadre geológico local, y con vistas a obtener unos datos que puedan enlazar con los resultados de los SPAC que más tarde se plantearán, se realiza una síntesis de la geología local basada en los resultados de Valverde (2010) en los análisis que realiza de las características de los suelos de Granada y del área metropolitana con fines orientativos para las cimentaciones de las edificaciones. Son muy interesantes los datos recogidos en los siguientes mapas (Fig. 2.3 y 2.4 ):

- El mapa de áreas litológico-geotécnicas (Fig. 2.3). Esta zonificación se ha establecido por Valverde (2010) mediante métodos cartográficos tradicionales a partir de sondeos, penetraciones dinámicas, calicatas y resultados de ensayos de laboratorio y comprobaciones de campo recogidos en una Base de Datos.

Para una mayor comprensión, el autor ha identificado las unidades litológico-geotécnicas mediante colores y números. El primer número ("1.2-3") corresponde a las Unidades Litológico-Geotécnicas o Zonas; el segundo ("1.2-3") a las 10 Subzonas, atendiendo a las características texturales predominantes y en algunos casos al origen del depósito; y el tercero ("1.2-3") a las 22 áreas diferenciadas en el mapa de la Vega de Granada en base a su litología, detallada en la Tabla 2.1. La Tabla 2.2.2 muestra los parámetros geotécnicos característicos de las diferentes Unidades litológicas.

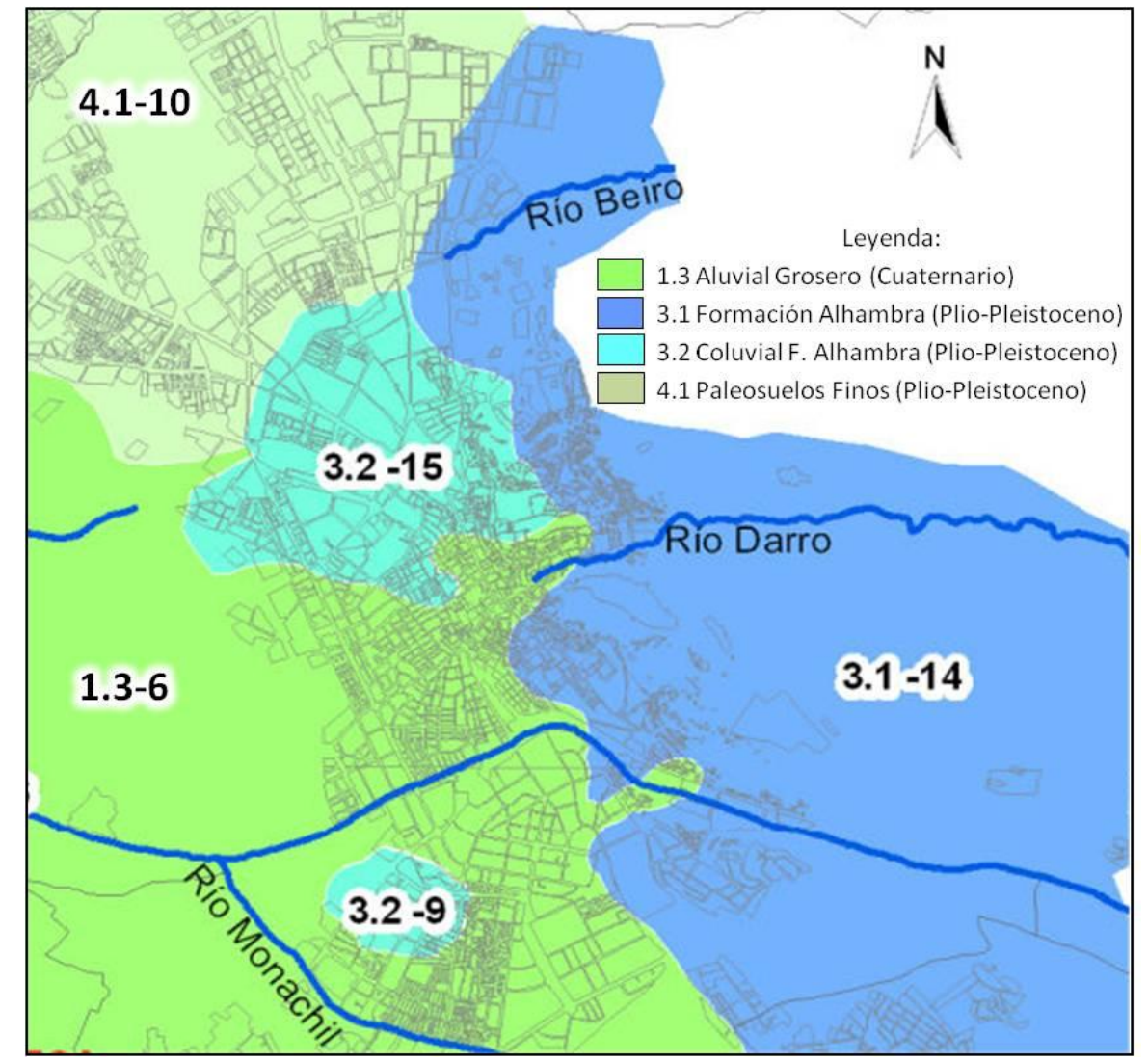

Figura 2.2. Mapa de áreas litológico-geotécnicas de la ciudad de Granada ( Valverde 2010). 
Tabla. 2.1 Distintas Zonas y Unidades Litológico-Geotécnicas (subzonas) con su descripción litológica

\begin{tabular}{|c|l|c|}
\hline Zonas & \multicolumn{1}{|c}{ Litología } & $\begin{array}{l}\text { Uds. Litológico- } \\
\text { Geotécnicas }\end{array}$ \\
\hline $\mathbf{6}$ & $\begin{array}{l}\text { Arenas, gravas, cantos y algún bolo con matriz limo-arenosa gris, } \\
\text { existiendo intercalaciones disimétricas, predominantes en los tres } \\
\text { primeros metros, de limos arenosos y de arenas finas y medias a } \\
\text { modo de bolsa o lentejón. }\end{array}$ & $\mathbf{1 . 3}$ \\
\hline $\mathbf{1 4}$ & $\begin{array}{l}\text { Conglomerados de arenas, gravas, cantos y algún bolo en matriz } \\
\text { arenosa con limos y arcillas de tonalidad rojiza. }\end{array}$ & $\mathbf{3 . 1}$ \\
\hline $\mathbf{9 1 5}$ & $\begin{array}{l}\text { Arcillas marrón rojizas con cantos; Limos arcillosos o niveles } \\
\text { granulares carbonatados de tonalidad crema; Arcillas marrones y } \\
\text { marrón rojizas; Niveles granulares grises. }\end{array}$ & $\mathbf{3 . 2}$ \\
\hline $\mathbf{1 0}$ & $\begin{array}{l}\text { Arcillas y arcillas limosas de tonalidades marrón, crema y marrón- } \\
\text { rojiza con material granular a modo de bolsa o lentejón, existiendo a } \\
\text { techo acumulaciones de carbonatos o arenas, gravas y cantos con } \\
\text { limos arcillosos marrones. }\end{array}$ & $\mathbf{4 . 1}$ \\
\hline
\end{tabular}

Tabla 2.2. Parámetros geotécnicos característicos correspondientes a las diferentes Unidades litológicas de Granada; Aluvial Grosero, Formación Alhambra, y Paleosuelos Finos

\begin{tabular}{|c|c|c|c|c|}
\hline $\begin{array}{l}\text { PARÁMETRO } \\
\text { (valor medio) }\end{array}$ & $\begin{array}{c}\text { ALUVIAL } \\
\text { GROSERO } \\
(1.3)\end{array}$ & $\begin{array}{c}\text { FORMACIÓN } \\
\text { ALHAMBRA } \\
\text { (3.1) }\end{array}$ & $\begin{array}{c}\text { FORMACIÓN } \\
\text { ALHAMBRA } \\
\text { (3.2) }\end{array}$ & $\begin{array}{l}\text { PALEOSUELOS } \\
\text { FINOS } \\
(4.1)\end{array}$ \\
\hline Nspt & 28 & 25 & 15 & 15 \\
\hline $\begin{array}{l}\text { Vs (velocidad de las ondas } \\
\text { de cizalla, } \mathrm{m} / \mathrm{s} \text { ) }\end{array}$ & 320 & 310 & 280 & 280 \\
\hline C (Coeficiente de Terreno) & 1,4 & 1,3 & 1,5 & 1,6 \\
\hline $\begin{array}{c}a_{c} \text { (aceleración sísmica de } \\
\text { cálculo) }\end{array}$ & 0,25 & 0,24 & 0,26 & 0,28 \\
\hline $\begin{array}{c}\text { S (Coeficiente de } \\
\text { Amplificacion, } \rho=1 \text { ) }\end{array}$ & 1,06 & 1,06 & 1,13 & 1,13 \\
\hline $\begin{array}{c}S \text { (Coeficiente de } \\
\text { Amplificacion, } \rho=1,3 \text { ) }\end{array}$ & 1,03 & 1,03 & 1,08 & 1,08 \\
\hline
\end{tabular}

Valverde, 2010

- El mapa de valores medios de Vs del material portante (Figura 2.4), en función de los valores de Nspt medio (tabla 2.2) más característico estimado a partir de la relación de Imai (1981) para todos los tipos de suelos:

$$
\mathrm{Vs}=91 \cdot \mathrm{N}^{0,337}
$$




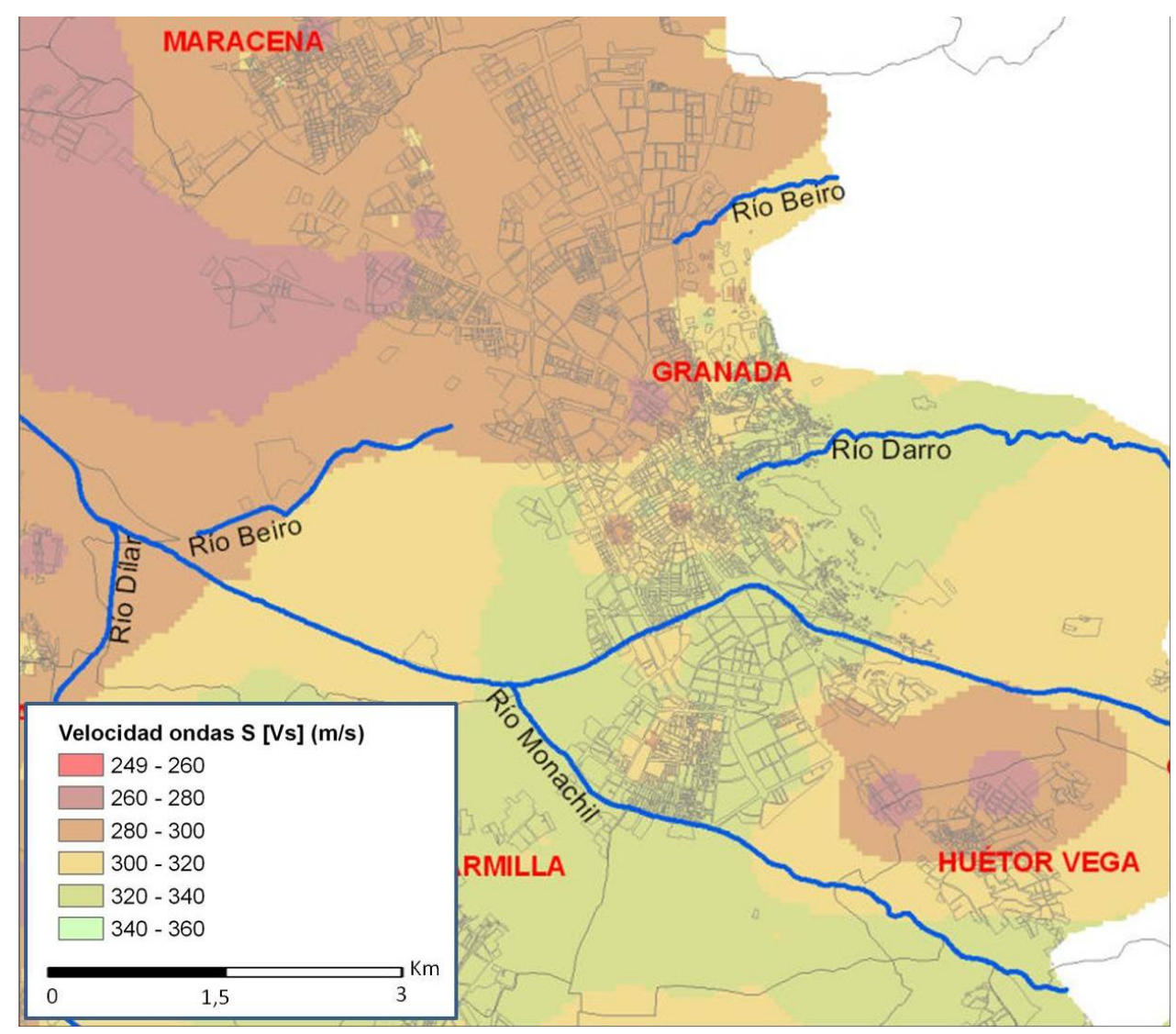

Figura. 2.3. Velocidades medias de las ondas sísmicas de cizalla (Vs) del material portante (Valverde, 2010) 


\section{3.- ADQUISICIÓN Y TRATAMIENTO DE DATOS}

\section{1.- El método de Autocorrelación Espacial (SPAC)}

El propósito del método SPAC (por su nombre en inglés Spatial Autocorrelations Method), propuesto por Aki (1957), es el de obtener la estructura de velocidades de un suelo a partir de las mediciones de microtremores. Para ello es necesario la medición simultánea de los microtremores en varias estaciones. El método necesita un mínimo de tres estaciones para ser aplicado. En síntesis el proceso consiste en el estudio de la curva de dispersión de las ondas Rayleigh y a partir de ahí se determina la estructura de velocidades.

\subsection{1.- Fundamento del método SPAC}

Aki (1957) propone el método a partir del estudio de los múltiples campos de ondas dispersivas que se propagan en todas las direcciones. La esencia del SPAC es que cuando los microtremores son medidos por un conjunto de estaciones equidistantes forman parejas de diferente azimut, es posible estimar la velocidad de fase de las ondas superficiales sin considerar su dirección de propagación. Esto es posible porque asumimos que el campo de ondas dispersivas se propaga a lo largo de una superficie libre y este es estacionario y estocástico en tiempo y espacio. Es muy importante verificar que estas condiciones se cumplan (Vázquez et al, 2011)

Así Aki consideró un campo de ondas estocástico formado por una superposición de varias ondas planas no polarizadas propagándose en varias direcciones en un plano horizontal, todas ellas propagándose con una velocidad de fase constante. El movimiento del suelo de dos localizaciones en la superficie $(x, y)$ y $(x+\xi, y+\eta)$ puede ser escrito como $u(x, y, t)$ y $u(x+\xi, y+\eta, t)$.

La función de autocorrelación espacial $\phi(\xi, \eta, t)$ para el caso 2D (ver Aki,1957) es definida como:

$$
\phi(\xi, \eta, t)=\overline{u(x, y, t) u(x+\xi, y+\eta, t)}
$$

donde $\overline{\mathrm{u}(\mathrm{t})}$ representa el valor medio de $\mathrm{u}(\mathrm{t})$ dentro del dominio del tiempo. 
Asumiendo que el campo de ondas está compuesto por ondas estacionarias estocásticas, y reemplazando $(\xi, \eta)$ por su componente polar, Aki (1957) muestra que el rango azimutal de la función espacial puede ser escrito como:

$$
\bar{\phi}(r)=\frac{1}{2 \pi} \int \phi(r, \psi) d \psi
$$

dónde $r$ y $\psi$ son las coordenadas polares definidas $\operatorname{como} \xi=r \cos \psi$ y $\eta=r \sin \psi$.

Además se demuestra que el rango azimutal de la función de autocorrelación $\phi(r)$ y el potencial de la densidad espectral $\phi(w)$ del campo de ondas $u$, donde $w$ es la frecuencia angular, se relacionan de la siguiente forma:

$$
\bar{\phi}(r)=\frac{1}{\pi} \int \phi(w) J_{0} \frac{w}{c} r d w
$$

en dónde Jo es la función de Bessel de orden cero.

Esta ecuación también se puede aplicar al caso de ondas dispersivas como muestra Aki (1957). Solo necesitamos sustituir $\mathrm{c}(\mathrm{w})$ por $\mathrm{c}$. Consideramos ahora que aplicamos un filtro de paso banda a la señal. La densidad espectral vendrá dada por:

$$
\bar{\phi}=P\left(w_{o}\right) \delta\left(w-w_{o}\right)
$$

donde $\mathrm{P}\left(\mathrm{w}_{0}\right)$ es el potencial espectral de densidad $w_{0}$. En este caso, el rango azimutal de la función de correlación espacial puede ser escrito (Aki, 1957) como:

$$
\bar{\phi}(r)=\bar{\phi}\left(r, w_{0}\right)=P\left(w_{0}\right) J_{0} \frac{w_{0}}{c\left(w_{0}\right)} r
$$

De acuerdo a Aki (1957), el coeficiente de autocorrelación puede ser definido como:

$$
\rho\left(r, w_{o}\right)=\frac{\phi\left(r, \psi, w_{o}\right)}{\phi\left(0, \psi, w_{o}\right)}
$$

Como $\mathrm{P}\left(\mathrm{w}_{0}\right)$ no depende de la posición podemos escribir (Aki,1957; Chávez-Garcia,2005):

$$
\rho\left(r, w_{o}\right)=J_{0} \frac{w_{0}}{c\left(w_{0}\right)} r
$$

El coeficiente SPAC $\rho(r, w)$ puede ser obtenido directamente en el dominio de la frecuencia usando la transformada de Fourier (FFT) de los microtremores observados:

$$
\rho(r, w)=\frac{1}{2 \pi} \int_{0}^{2 \pi} \frac{\operatorname{Re}_{c x}(w, r, \theta)}{\sqrt{S_{c}(w) \cdot S_{x}(w, r, \theta)}} d \theta
$$

donde Re es la parte real del argumento complejo, $S c(w)$ y $S x(w, r, \theta)$ son los potenciales de las densidades espectrales de los microtremores en los lugares $C$ y $X$ respectivamente, y $S c x$ ( $w, r$, $\theta$ ) es el espectro cruzado (cross spectrum) entre el movimiento del suelo de los dos lugares.

Con el coeficiente SPAC $\rho(r, w)$, la velocidad de fase es obtenida para cada frecuencia a través del argumento de la función de Bessel de la ecuación (7), donde c(f) es la velocidad de fase para la frecuencia $f$. Obtendremos el valor óptimo para c(f) mediante una búsqueda en 
cuadrícula. Más información de ello se puede encontrar en Midorikawa etal. $(1998,2004)$, en Flores-Estrella (2004) y Malischewsky (1987).

\section{2.- Características de las medidas de SPAC en diferentes puntos de Granada}

\subsection{1.- Características de los dispositivos de registro simultáneo de ruido}

Para nuestro estudio se utilizaron los resultados de una serie de medidas de ruido ambiental que se realizaron en la ciudad de Granada y que posteriormente fueron tratados por el método de la Autocorrelación Espacial (SPAC) para obtener el modelo de terreno.

Las medidas se realizaron en nueve lugares diferentes con suelos de diferentes características. A partir de las medidas de ruido se trataron los datos mediante el método SPAC con el que se obtuvieron las curvas de dispersión de las ondas Rayleigh. A partir de ese momento se obtuvieron los perfiles de velocidades de las ondas $\mathrm{S}$ mediante la inversión.

Las componentes verticales del movimiento del suelo, excitadas por el ruido ambiental, fueron medidas con un dispositivo (array) en forma circular (Figura 3.1) formado por cinco sensores de alta sensibilidad VSE-15D (Figura 3.2) que rodeaban a un sensor central con las mismas características, todo ellos conectados a un digitalizador SPC-35. El radio fue variando desde los 3 hasta los 30 metros. Se usaron varios radios dependiendo del espesor de sedimentos que se preveía existiera bajo los sensores.

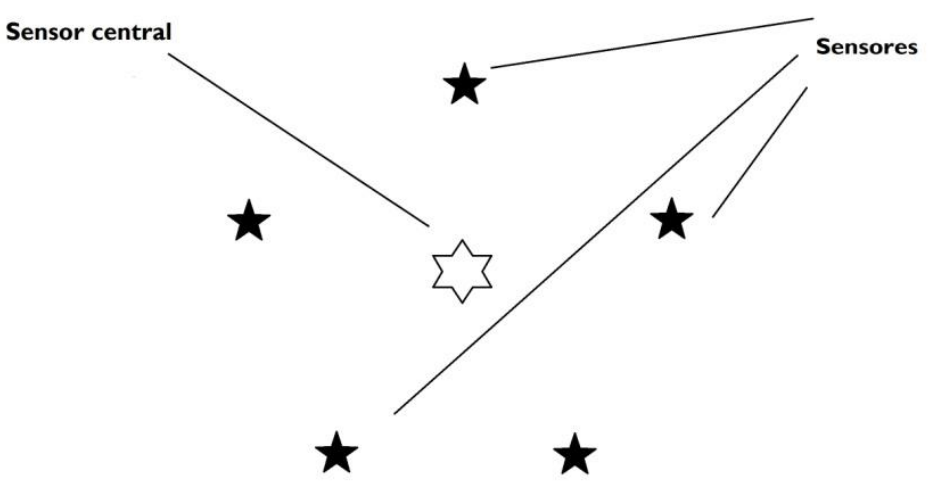

Figura. 3.1 Esquema de colocación de los sensores durante la medición del ruido ambiental

Las mediciones duraron unos 30 minutos en donde las señales se muestraban 200 veces por segundo. Todas las grabaciones fueron analizadas usando la implementación del método SPAC. Las correlaciones cruzadas entre las grabaciones de los sensores colocados en el círculo y el 
sensor central fueron calculadas en el dominio de la frecuencia obteniéndose el coeficiente de correlación $\rho(f, R)$.

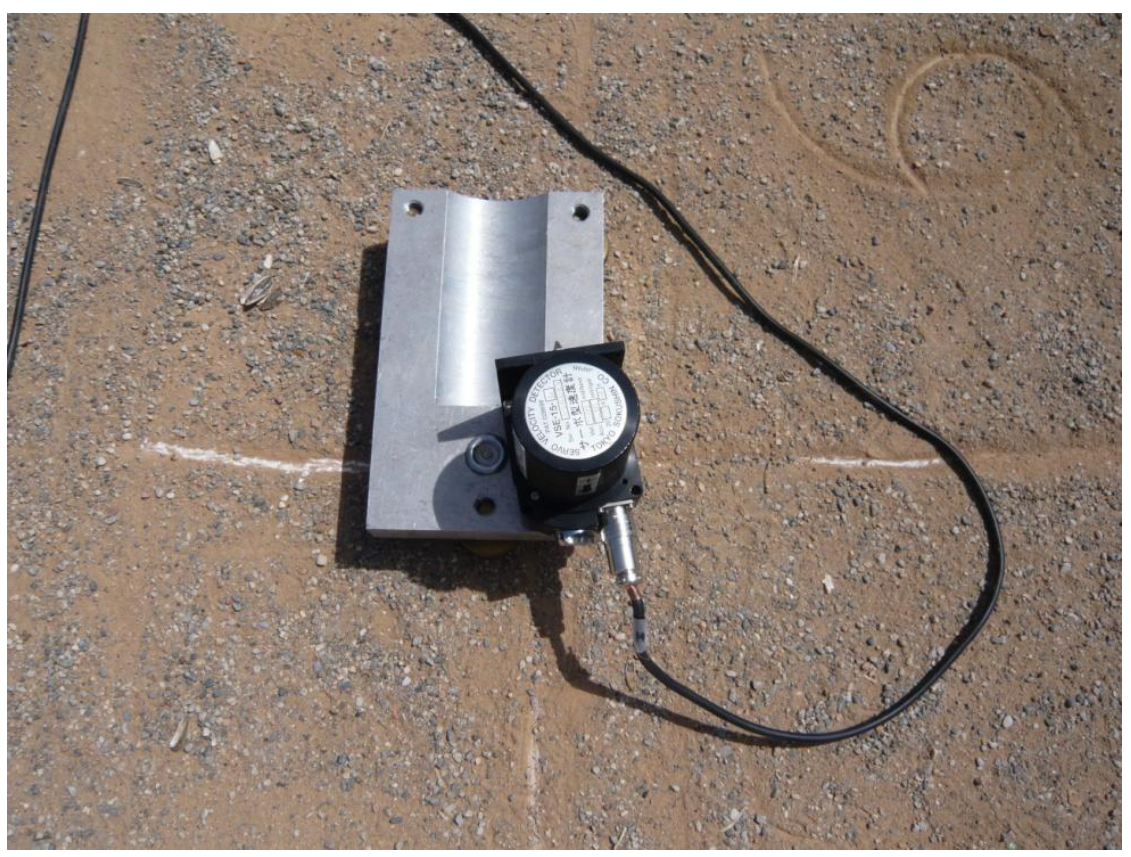

Figura. 3.2 Detalle de uno de los sensores utilizados durante las mediciones de ruido

Posteriormente el rango azimutal fue dividido para la autocorrelación con el sensor central. Finalmente la velocidad de fase de las ondas Rayleigh $\mathrm{C}(\mathrm{f})$ fue calculada para cada frecuencia $f$ usando la expresión:

$$
\rho(\mathrm{f}, \mathrm{R})=\mathrm{J}_{0}\left(\frac{2 \pi \mathrm{f}}{\mathrm{c}(\mathrm{f})} \mathrm{R}\right)
$$

donde Jo representa la función de Bessel de orden cero y $\mathrm{R}$ el radio entre los sensores del círculo y el sensor central.

\subsection{2.- Puntos de estudio}

Para la aplicación del método SPAC se eligieron una serie de emplazamientos con dimensiones suficientes (círculos con un radio $R \geq 30 \mathrm{~m}$ ) en los que fuera posible instalar los equipos necesarios de los dispositivos o antenas (arrays). Además se buscó que dichos puntos de medida estuvieran distribuidos por la mayor parte de la ciudad.

La localización de los 13 puntos de SPAC, cuyos datos se utilizaron para obtener la estructura Vs superficial, se pueden observar en la figura 3.1 y los nombres y ubicación en la tabla 3.1. 


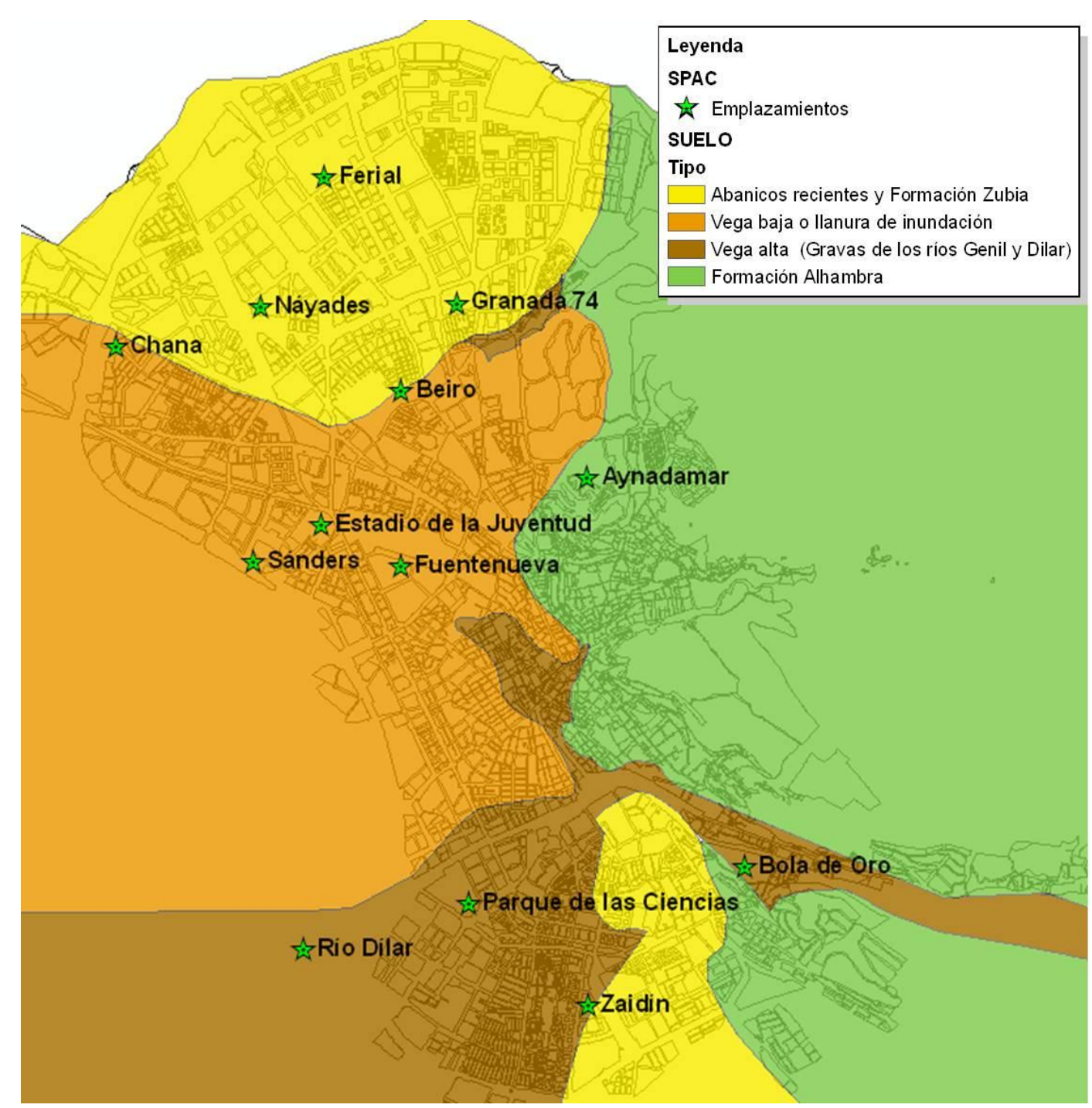

Figura 3.1. Emplazamiento de los puntos de medidas de SPAC y principales unidades sismo-geológicas de la ciudad de Granada.

Tabla. 3.1 Localización de los puntos de SPAC

\begin{tabular}{|c|c|c|}
\hline Array & Nombre & Localización \\
\hline GR1 & Granada1 & Aynadamar \\
GR2 & Granada1B & Zaidin \\
GR3 & Granada2 & Beiro \\
GR4 & Granada2B & Bola de Oro \\
GR5 & Granada3 & Fuentenueva \\
GR6 & Granada3B & Est. de la Juventud \\
GR7 & Granada4 & Chana \\
GR8 & Granada4B & Náyades \\
GR9 & & Par. de las Ciencias \\
GR10 & Granada5 & Granada-74 \\
GR11 & Granada6 & Ferial \\
GR12 & Granada5 & Antigua Sanders \\
GR13 & Granada8 & Vega \\
\hline
\end{tabular}




\subsection{3.- Medidas de ruido obtenidas}

Tras la realización de las mediciones de ruido en los puntos anteriormente mencionados, en las figuras 3.2 a 3.3 se exponen muestras de los registros que se obtuvieron de microtremores en algunas estaciones y en el Apéndice 1 las de todos.
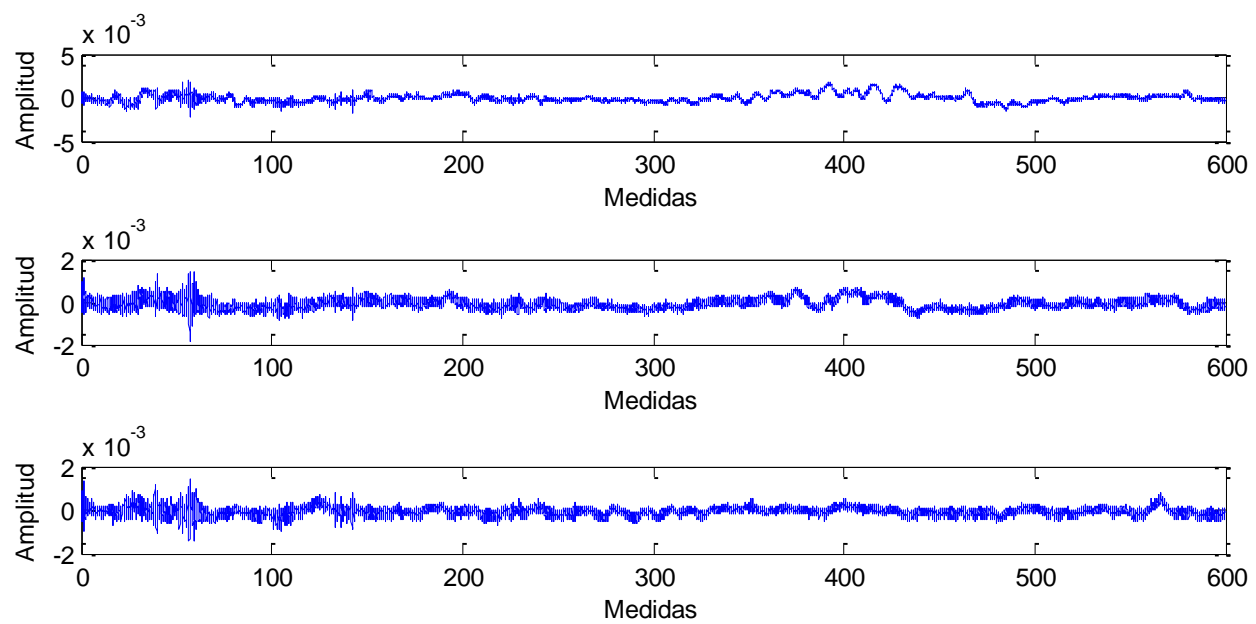

Figura 3.2. Ejemplo medidas de microtremores en el array de Ainadamar.
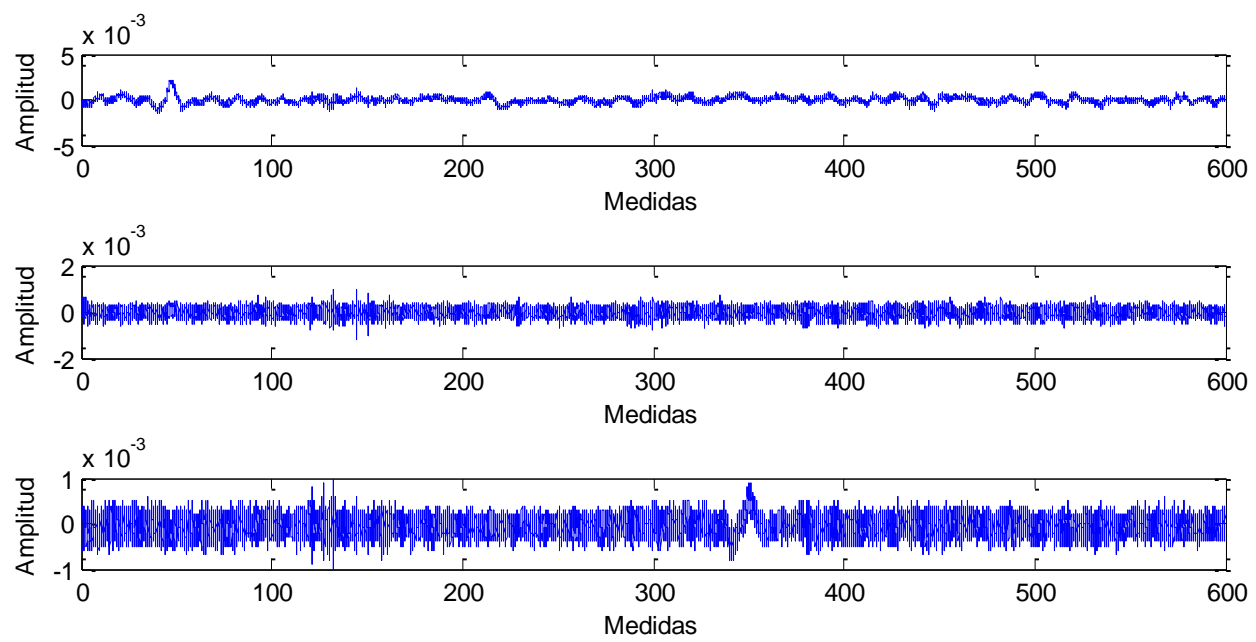

Figura 3.3. Ejemplo medidas de microtremores en el array de Beiro. 


\section{3.- Resultados obtenidos de la aplicación del método SPAC a Granada}

\subsubsection{Determinación de la $\mathrm{V}_{\mathrm{S}}{ }^{30}$}

La velocidad media de las ondas de cizalla de los últimos 30 metros $\left(\mathrm{V}_{\mathrm{S}}{ }^{30}\right)$ es una expresión sintética de las propiedades dinámicas de un perfil de suelo, idónea para cuantificar la influencia de las condiciones del lugar en el espectro de respuesta elástica. La $V_{s}{ }^{30}$ ha sido adoptada en varios códigos sísmicos (p.e. Uniform Building Code, UBC; NEHRP 2003; Eurocode8, EC8; NCSE-02) como parámetro representativo de las características del comportamiento sísmico del suelo. Además de emplearse para la elaboración de códigos sísmicos (Dobry et al, 2000) también se utiliza para la evaluación de los efectos de sitio a partir de la clasificación regional de suelos (Alcalá et al., 2002; Ismet et al., 2006).

Para calcular el valor medio ponderado de $\mathrm{V}_{\mathrm{S}}^{30}$ se ha utilizado el EC8:

$$
V_{S}^{30}=\frac{30}{\sum_{i=1}^{n} \frac{h_{i}}{V_{i}}}
$$

donde hi y Vi representan el espesor (en $\mathrm{m}$ ) y la velocidad de ondas de cizalla (en $\mathrm{m} / \mathrm{s}$ ) de la capa i de un total de $n$ existentes en los últimos $30 \mathrm{~m}$.

Las $\mathrm{V}_{\mathrm{S}}{ }^{30}$ medias obtenidas se representan en la tabla 3.2. Dichos valores resultarán fundamentales para la caracterización geotécnica del suelo y posteriormente los resultados de la velocidad de las ondas S. En la tabla 3.3 se muestran, además de las características de las configuraciones utilizadas en los arrays, las curvas de dispersión, el coeficiente de correlación, el rango de valores para la velocidad de cizalla o Vs y la Vs30 calculada para cada sitio.

Tabla 3.2 Valores $\mathrm{Vs}^{30}$ de las principales unidades litológicas de la ciudad de Granada y comparación con las clases de suelo propuestas por la NCSE-02 (Navarro et al, 2011).

\begin{tabular}{|c|c|c|c|c|}
\hline Unidad geológica & Litología & VS 30 (m/s) & $\begin{array}{c}\text { VS } \mathbf{3 0}(\mathrm{m} / \mathrm{s}) \\
\text { NCSE-02 }\end{array}$ & $\begin{array}{c}\text { Suelo de } \\
\text { NCSE-02 }\end{array}$ \\
\hline $\begin{array}{c}\text { Formación Alhambra } \\
\text { (transición) }\end{array}$ & $\begin{array}{c}\text { Arena, grava con limos marrón. } \\
\text { Capas cementadas por carbonatos. }\end{array}$ & $519 \pm 08$ & $400-750$ & Tipo II \\
\hline $\begin{array}{c}\text { Aluvial antiguo } \\
\text { Aluvial reciente I }\end{array}$ & $\begin{array}{c}\text { Arcillas y arenas limosas con capas } \\
\text { de gravas menores }\end{array}$ & $328 \pm 16$ & $200-400$ & Tipo III \\
\hline Aluvial reciente II & $\begin{array}{c}\text { Gravas y arenas con intercalaciones } \\
\text { de arcillas rojas }\end{array}$ & $463 \pm 40$ & $400-750$ & Tipo II \\
\hline
\end{tabular}


Tabla 3.3 Características de las configuraciones utilizadas, curvas de dispersión, rango de valores de velocidad de cizalla y Vs30 (Navarro et al 2011).

\begin{tabular}{|c|c|c|c|c|c|c|}
\hline Array & Localización & Radio $(\mathrm{m})$ & $\Delta \mathrm{f}(\mathrm{Hz})$ & $\Delta \mathrm{cR}(\mathrm{m} / \mathrm{s})$ & $\Delta V \mathrm{VS}(\mathrm{m} / \mathrm{s})$ & VS $30(\mathrm{~m} / \mathrm{s})$ \\
\hline GR1 & Aynadamar & $4-8-15-27$ & $6,5-28,9$ & $349-608$ & $304-690$ & 526 \\
\hline GR2 & Zaidin & $3-6-12-24$ & $5,0-17,9$ & $239-573$ & $204-679$ & 407 \\
\hline GR3 & Beiro & $4-8-16-30$ & $4,3-14,9$ & $272-526$ & $178-648$ & 341 \\
\hline GR4 & Bola de Oro & $3-6-12-24$ & $7,0-21,9$ & $310-678$ & $268-814$ & 511 \\
\hline GR5 & Fuentenueva & $4-8-16-30$ & $3,7-18,2$ & $291-619$ & $207-696$ & 326 \\
\hline GR6 & Est. de la Juventud & $3-6-12-24$ & $2,5-23,9$ & $228-415$ & $239-459$ & 330 \\
\hline GR7 & Chana & $3-6-12-25$ & $3,0-22,9$ & $254-478$ & $242-549$ & 343 \\
\hline GR8 & Náyades & $3-6-12-24$ & $2,6-11,3$ & $236-413$ & $180-469$ & 294 \\
\hline GR9 & Par. de las Ciencias & $4-8-15-30$ & $5,5-31,0$ & $329-682$ & $293-784$ & 496 \\
\hline GR10 & Granada-74 & $4-8-15-30$ & $3,5-24,8$ & $342-598$ & $278-667$ & 462 \\
\hline GR11 & Ferial & $5-11-22$ & $3,2-33,5$ & $271-480$ & $281-523$ & 416 \\
\hline GR12 & Sanders & $6-12-23$ & $3,4-18,8$ & $284-609$ & $238-602$ & 316 \\
\hline GR13 & Vega & $4-8-15-30$ & $5,3-29,8$ & $355-708$ & $326-794$ & 468 \\
\hline
\end{tabular}

\subsubsection{Curvas de dispersión de ondas Rayleigh obtenidas.}

En la figura 3.4 se representan las curvas de dispersión de ondas Rayleih obtenidas en diferentes puntos.
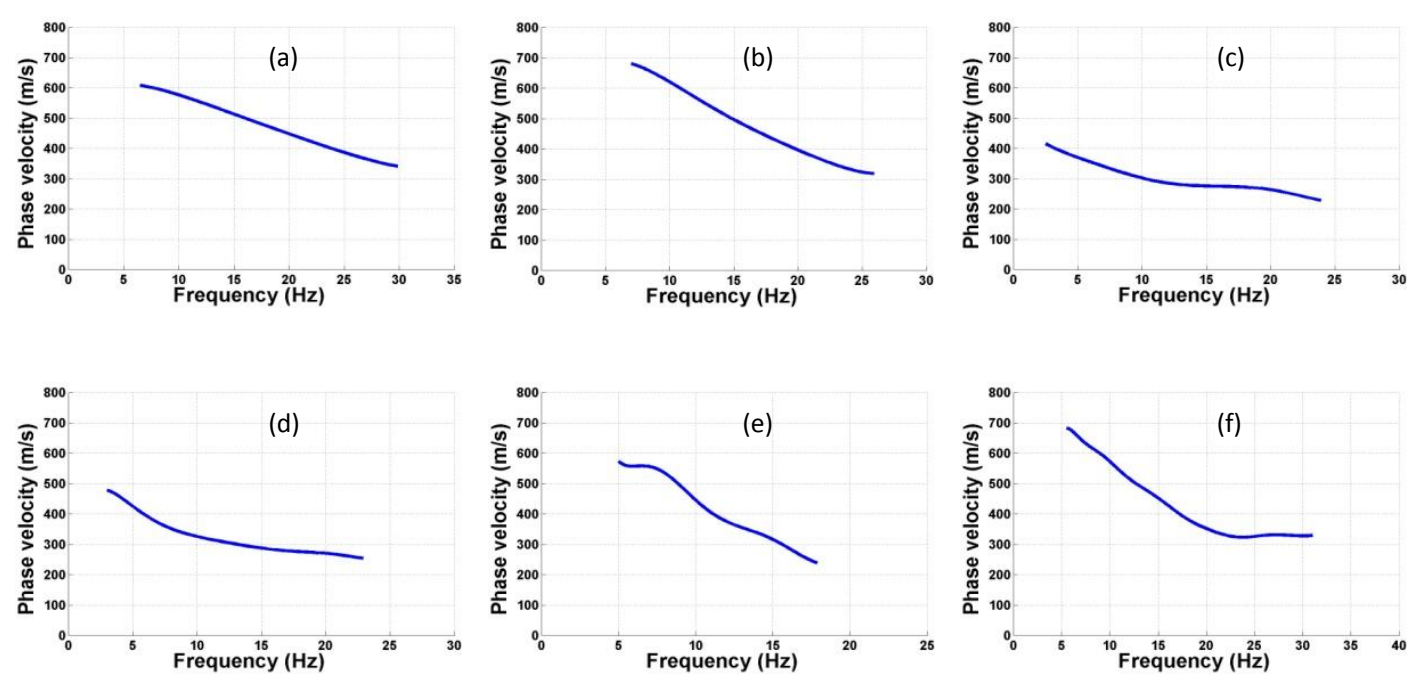

Fig. 3. 4. Algunos ejemplos de la velocidad de fase para las ondas Rayleigh obtenidas para el modelo de dispersión. (a) Aynadamar ; (b) Bola de Oro ; (c) Estadio de la Juventud ; (d) Chana (e) Zaidin ; (f) Parque de las Ciencias 


\subsubsection{Estructuras superficiales $\mathrm{V}_{\mathrm{S}}$ obtenidas.}

Así y finalmente tras aplicar el método de inversión (Navarro et al,1.997) se obtuvieron los resultados del perfil de velocidades de ondas $\mathrm{S}$ para cada uno de los puntos en estudio. $\mathrm{A}$ continuación se exponen dichos resultados en las tablas 3.4 a 3.17 .

Tabla 3.4. Estructura del suelo en Aynadamar

\begin{tabular}{|c|c|}
\hline Profundidad $(\mathrm{m})$ & Velocidad de las ondas S $(\mathrm{m} / \mathrm{s})$ \\
\hline 5,65 & 318,37 \\
\hline 16,71 & 549,02 \\
\hline 28,19 & 677,53 \\
\hline 39,08 & 738,04 \\
\hline
\end{tabular}

Tabla 3.5. Estructura del suelo en Zaidín

\begin{tabular}{|c|c|}
\hline Profundidad $(\mathrm{m})$ & Velocidad de las ondas S $(\mathrm{m} / \mathrm{s})$ \\
\hline 3,14 & 141,19 \\
\hline 8,84 & 316,59 \\
\hline 21,13 & 498,72 \\
\hline 33,50 & 773,43 \\
\hline 44,77 & 726,73 \\
\hline
\end{tabular}

Tabla 3.6. Estructura del suelo en Beiro

\begin{tabular}{|c|c|}
\hline Profundidad $(\mathrm{m})$ & Velocidad de las ondas S $(\mathrm{m} / \mathrm{s})$ \\
\hline 3,50 & 226,58 \\
\hline 10,74 & 290,75 \\
\hline 22,60 & 436,34 \\
\hline 36,12 & 500,28 \\
\hline 45,86 & 540,08 \\
\hline
\end{tabular}

Tabla 3.7. Estructura del suelo en Bola de Oro

\begin{tabular}{|c|c|}
\hline Profundidad $(\mathrm{m})$ & Velocidad de las ondas S $(\mathrm{m} / \mathrm{s})$ \\
\hline 3,70 & 250,51 \\
\hline 8,85 & 397,85 \\
\hline 19,79 & 604,86 \\
\hline 30,99 & 744,65 \\
\hline 43,09 & 842,37 \\
\hline
\end{tabular}

Tabla 3.8. Estructura del suelo en Fuentenueva

\begin{tabular}{|c|c|}
\hline Profundidad $(\mathrm{m})$ & Velocidad de las ondas S (m/s) \\
\hline 11,64 & 250,51 \\
\hline 23,18 & 397,85 \\
\hline 32,65 & 604,86 \\
\hline 54,88 & 744,65 \\
\hline
\end{tabular}


Tabla 3.9. Estructura del suelo en Fuentenueva 2

\begin{tabular}{|c|c|}
\hline Profundidad $(\mathrm{m})$ & Velocidad de las ondas S (m/s) \\
\hline 1,51 & 268,45 \\
\hline 9,59 & 301,83 \\
\hline 22,57 & 424,38 \\
\hline 32,50 & 371,89 \\
\hline 43,44 & 423,66 \\
\hline
\end{tabular}

Tabla 3.10. Estructura del suelo en Estadio

\begin{tabular}{|c|c|}
\hline Profundidad $(\mathrm{m})$ & Velocidad de las ondas S (m/s) \\
\hline 3,64 & 219,50 \\
\hline 11,69 & 302,51 \\
\hline 21,97 & 369,52 \\
\hline 32,47 & 422,25 \\
\hline 46,11 & 408,47 \\
\hline
\end{tabular}

Tabla 3.11. Estructura del suelo en Chana

\begin{tabular}{|c|c|}
\hline Profundidad $(\mathrm{m})$ & Velocidad de las ondas S (m/s) \\
\hline 3,44 & 231,17 \\
\hline 12,04 & 308,83 \\
\hline 22,29 & 396,87 \\
\hline 32,16 & 468,62 \\
\hline 43,67 & 49,78 \\
\hline
\end{tabular}

Tabla 3.12. Estructura del suelo en Náyades

\begin{tabular}{|c|c|}
\hline Profundidad $(\mathrm{m})$ & Velocidad de las ondas S (m/s) \\
\hline 4,93 & 198,77 \\
\hline 12,65 & 258,51 \\
\hline 24,35 & 422,40 \\
\hline 30,76 & 328,55 \\
\hline 41,60 & 390,86 \\
\hline
\end{tabular}

Tabla 3.13. Estructura del suelo en Parque de las ciencias

\begin{tabular}{|c|c|}
\hline Profundidad $(\mathrm{m})$ & Velocidad de las ondas S (m/s) \\
\hline 3,32 & 319,20 \\
\hline 9,87 & 347,41 \\
\hline 21,38 & 646,31 \\
\hline 32,73 & 652,21 \\
\hline 43,39 & 718,57 \\
\hline
\end{tabular}


Tabla 3.14. Estructura del suelo en Granada 74

\begin{tabular}{|c|c|}
\hline Profundidad $(\mathrm{m})$ & Velocidad de las ondas S (m/s) \\
\hline 4,56 & 260,61 \\
\hline 17,17 & 471,04 \\
\hline 31,93 & 629,83 \\
\hline 54,52 & 658,25 \\
\hline
\end{tabular}

Tabla 3.15. Estructura del suelo en Ferial

\begin{tabular}{|c|c|}
\hline Profundidad $(\mathrm{m})$ & Velocidad de las ondas S (m/s) \\
\hline 2,14 & 169,88 \\
\hline 4,88 & 341,59 \\
\hline 8,99 & 394,93 \\
\hline 18,95 & 458,58 \\
\hline 30,23 & 530,99 \\
\hline 39,26 & 463,76 \\
\hline 55,35 & 534,01 \\
\hline
\end{tabular}

Tabla 3.16. Estructura del suelo en Sanders

\begin{tabular}{|c|c|}
\hline Profundidad $(\mathrm{m})$ & Velocidad de las ondas S (m/s) \\
\hline 2,13 & 169,38 \\
\hline 5,01 & 288,58 \\
\hline 9,98 & 366,37 \\
\hline 19,82 & 312,39 \\
\hline 30,83 & 487,16 \\
\hline 41,59 & 412,65 \\
\hline 64,11 & 481,53 \\
\hline
\end{tabular}

Tabla 3.17. Estructura del suelo en Vega

\begin{tabular}{|c|c|}
\hline Profundidad $(\mathrm{m})$ & Velocidad de las ondas S (m/s) \\
\hline 5,93 & 353,35 \\
\hline 15,33 & 434,39 \\
\hline 31,01 & 63,88 \\
\hline 52,55 & 653,64 \\
\hline
\end{tabular}




\section{4.- AMPLIFICACIÓN SÍSMICA}

\section{1.- Introducción}

La amplificación sísmica y el patrón de daño observado durante sismos de gran magnitud presentan una importante variabilidad de un lugar a otro, aun en distancias relativamente cortas, siendo esto explicado por las variaciones locales en el tipo de suelo y la topografía.

Este fenómeno de amplificación local de las ondas sísmicas asociadas principalmente a las propiedades geotécnicas del subsuelo y profundidad del basamento rocoso se suele denominar "efecto de sitio" o simplemente "amplificación de suelos".

De este modo, el daño causado por sismos es fuertemente dependiente de las condiciones locales del terreno y por tanto la caracterización del subsuelo es un tema de considerable interés en la ingeniería.

\section{2.- Efecto de sitio}

La modificación de la señal sísmica debida a la influencia de las condiciones geológicas y topográficas durante un terremoto se conoce como efecto de sitio como ya se ha mencionado. Dicha modificación puede consistir en la amplificación de la señal así como una mayor duración de la misma y la variación de su contenido frecuencial.

Así, frente a un movimiento sísmico las capas de suelo actúan como filtros de frecuencias, de manera que modifican la energía que transmiten al resto de la estructura. Se ha observado que los depósitos de suelo amplifican el movimiento o señal de entrada cuando las frecuencias predominantes son bajas (periodos altos) mientras que los suelos rígidos lo hacen con señales cuyas frecuencias son predominantemente altas (periodos bajos) (Trigo Salas, 2007).

Por otra parte, si el suelo donde se apoye la cimentación es blando se puede producir el fenómeno de interacción suelo-estructura que consiste en que la flexibilidad del suelo influye en el movimiento de la estructura y la estructura influye en el movimiento del suelo. Dicha interacción puede ser importante en estructuras rígidas y de gran masa como reactores nucleares o grandes infraestructuras civiles como puentes o presas. 
Asimismo también se pueden producir otros fenómenos que alteren la respuesta de la estructura como las roturas del terreno, deslizamientos, caídas de roca o licuefacción. Estos son conocidos como efectos inducidos y provocan la pérdida de capacidad portante del terreno o son causa directa de daño estructural (Rodriguez Ortiz, Geotecnia)

Por estos motivos la identificación de las frecuencias predominantes y la cuantificación de la amplificación de la señal sísmica constituyen aspectos de máximo interés en el campo de la ingeniería sísmica.

Existen diferentes técnicas para hallar e identificar ambos parámetros, en este trabajo se recurrirá a la técnica de Nakamura (1989) y a la técnica de Tsai (1969). La primera de tipo semiempírico y la última de tipo analítico.

\section{3.- Respuesta sísmica de los suelos}

\subsection{1.- Propiedades dinámicas de los suelos}

La amplificación del movimiento en el suelo está determinada por sus propiedades dinámicas. Dentro de éstas, la rigidez y el amortiguamiento del suelo constituyen las propiedades claves para el estudio de la respuesta de sitio. El módulo de corte $\mathrm{G}$ representa una buena medida de la rigidez del suelo, el cual se calcula por medio de la velocidad de las ondas sísmicas. Por su parte, la razón de amortiguamiento, $\xi$, representa la capacidad que tiene el suelo para disipar la energía. Otras propiedades pero de menor importancia son la densidad y el coeficiente de Poisson. (Rodriguez Salas, Curso Aplicado de Cimentaciones).

\subsection{2.- Efectos del suelo en superficie}

Como se mencionó anteriormente, la amplificación del movimiento del suelo es el responsable del daño ocurrido en áreas constituidas por depósitos de sedimentos blandos y poco compactos.

Normalmente para terremotos de pequeña magnitud, la amplificación es típicamente mayor en áreas ubicadas en una cierta distancia epicentral donde sería esperable que las ondas sísmicas redujesen la amplitud debido a los efectos de atenuación en la propagación de la señal sísmica.

Dos mecanismos contribuyen a los efectos de amplificación de la señal en el subsuelo: la amplificación geométrica y la amplificación dinámica.

\section{- Amplificación geométrica.}

Corresponde a los efectos de amplificación debido al contraste de impedancias entre dos medios en contacto. Para sedimentos el contraste de impedancias se expresa como: 


$$
I_{C}=\frac{\rho_{B} v_{B}}{\rho_{S} v_{S}} \operatorname{con} v_{B}>v_{S}
$$

donde el subíndice B representa al substrato rocoso y $\mathrm{S}$ se refiere a la capa de sedimentos superior y donde $\rho$ es la densidad y $v$ la velocidad de las ondas sísmicas.

En materiales jóvenes y poco consolidados el contraste de impedancias con el basamento es mayor, lo que provoca un mayor nivel de amplificación de la señal sísmica. Asimismo se produce el atrapamiento de las ondas sísmicas dentro de un nivel con baja impedancia y esto provoca la amplificación en la banda de las frecuencias características. La velocidad de las ondas sísmicas $\mathrm{S}$ en el suelo es menor que en el basamento. Entonces cuando la capa de sedimentos está situada sobre un basamento rocoso duro, aumenta el contraste de impedancias. Como se ha mencionado los depósitos del suelo se comportan como filtros reduciendo la energía de alta frecuencia (o corto periodo) pero la amplificación de las bajas frecuencias puede causar daño potencial a estructuras que tienen frecuencias naturales bajas, como por ejemplo edificios altos y puentes.

\section{- Amplificación dinámica}

La teoría clásica de amplificación de suelos predice que al considerar una capa de suelo horizontal infinita de espesor $\mathrm{H}$ uniforme e isótropa y con un comportamiento lineal elástico sobre sustrato rocoso, también horizontal sometido a un campo de propagación de ondas SH incidiendo verticalmente, la función de amplificación del suelo será como la que se muestra en la fig. 4.1

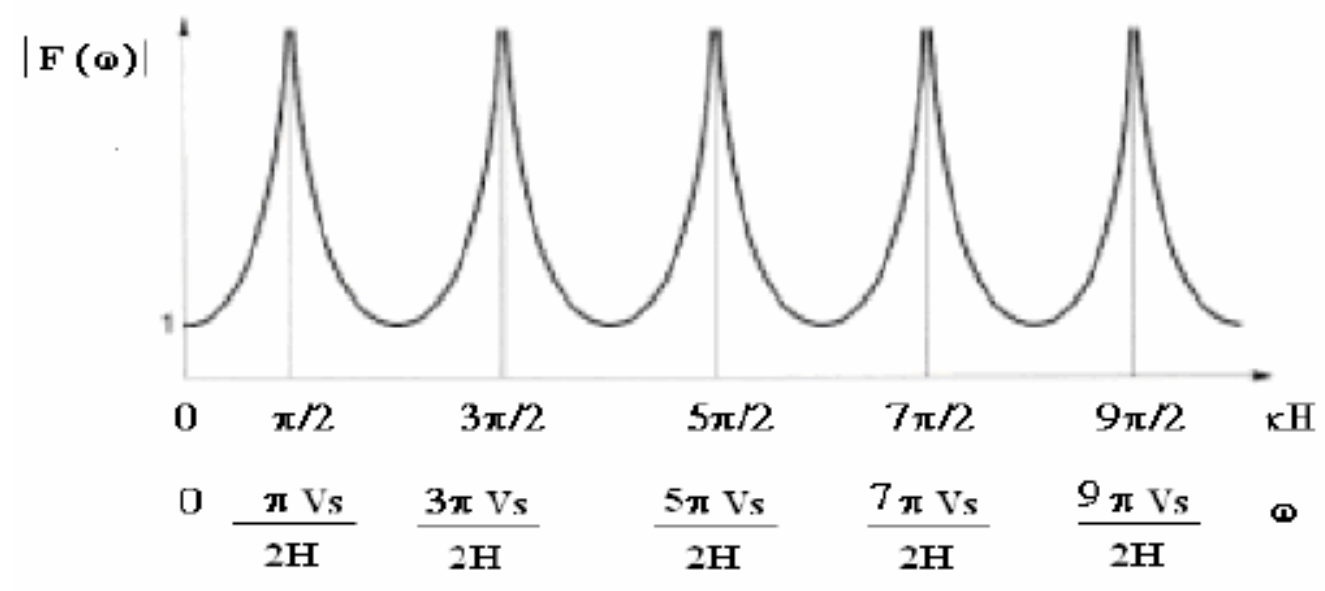

Figura 4.1. Ejemplo de función de amplificación de un suelo uniforme sobre capa de basamento rígida con un coeficiente de amortiguación nulo.

Si el suelo no fuese capaz de amortiguar el movimiento ondulatorio se produciría una amplificación infinita (resonancia) para frecuencias que tienden a $\pi / 2+n \pi$. Sin embargo gracias a la capacidad del material para amortiguar el movimiento se producen dichas amplificaciones pero no de manera infinita. 


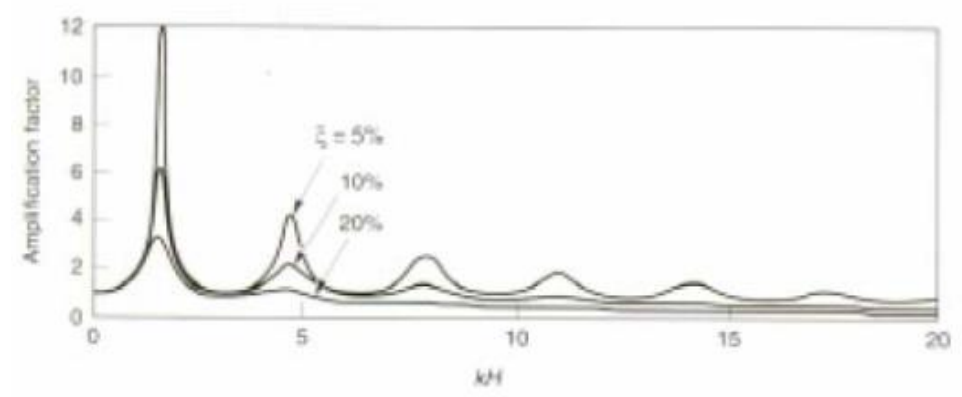

Figura 4.2. Ejemplo de función de amplificación de un suelo uniforme sobre capa de basamento rígido con un coeficiente de amortiguamiento diferente de cero.

Las frecuencias a las que se dan los máximos locales se conocen con el nombre de frecuencias naturales del suelo y dependen de la velocidad de ondas sísmicas $\mathrm{S}$ y del espesor del sedimento $H$, según:

$$
\omega_{n}=\frac{V s}{H}\left(\frac{\pi}{2}+n \pi\right) \quad \text { con } \mathrm{n}=0,1,2, \ldots
$$

La frecuencia fundamental es la frecuencia más baja conocida como $\omega_{0}=\pi \mathrm{V} s / 2 \mathrm{H}$ cuyo periodo asociado se conoce como el periodo característico del sitio.

Esta amplificación también se conoce como efecto de resonancia. Dicho efecto considera la diferencia entre la frecuencia de las ondas sísmicas y la frecuencia natural del depósito sedimentario.

Si la frecuencia de la onda sísmica es aproximadamente igual a la frecuencia natural del depósito se produce amplificación incrementándose la amplitud del movimiento del suelo significativamente.

Así los fenómenos que contribuyen a la amplificación de la señal sísmica están relacionados directamente con la velocidad de las ondas sísmicas de corte $\mathrm{S}$ en el basamento. Cuando una onda elástica se propaga a través de un material, el flujo de energía transmitida está definido por $\rho V s$. Este flujo de energía permanece constante y en consecuencia las amplitudes del movimiento del suelo en materiales con baja velocidad de propagación son mayores dado que la velocidad de la partícula u y la velocidad de las ondas $S$ son inversamente proporcionales. En consecuencia, materiales como arena suelta o arcilla blanda amplifican el movimiento del suelo significativamente. 


\section{METOdOLOGÍA PARA EL CÁlCULO DEL EFECTO DE SITIO}

\subsection{Método HV de Nakamura}

\subsubsection{Fundamento teórico}

El método de la razón espectral H/V o de Nakamura (1989), se basa en la relación de los espectros de Fourier de las componentes horizontal y vertical de los registros de microtemblores en superficie. Este método ha sido ampliamente difundido y utilizado en los últimos años por la fiabilidad de los resultados que de él se obtienen además de la comodidad y rapidez que suponen su ejecución.

Se basa en la suposición de que la razón $\mathrm{H} / \mathrm{V}$ o razón espectral corresponde a la función de transferencia de los niveles superficiales sometidos a movimientos horizontales. Para llegar a esto hay que tomar las siguientes hipótesis:

- Los microtremores están compuestos por ondas internas y por ondas superficiales Rayleigh inducidas por fuentes artificiales (tráfico, maquinaria, etc.) Dichas ondas superficiales se consideran perturbaciones que es necesario eliminar

- Las componentes vertical y horizontal son similares en la base rocosa

- Las ondas Rayleigh sólo afectan a las componentes horizontales y verticales en superficie mientras que no afectan las componentes en base rocosa

- Se considera además que la componentes horizontal de los microtremores es amplificada por la multirreflexión de la onda $S$ y la componente vertical por multirreflexión de la onda $\mathrm{P}$

- Los registros son amplificados por las capas de suelo blando que existan sobre la base rocosa

- El efecto de las ondas Rayleigh es visible en el movimiento vertical fundamentalmente.

Así pues el método que propone Nakamura (1989) lo que intenta es eliminar el efecto de las ondas Rayleigh para poder describir la función de transferencia de las ondas internas.

De esta forma la función de transferencia $\mathrm{S}_{\mathrm{T}}$ es definida de la siguiente forma:

$$
S_{T}=\frac{S_{H S}}{S_{H B}}
$$


Donde $\mathrm{S}_{\mathrm{HS}}$ y $\mathrm{S}_{\mathrm{HB}}$ corresponden a los espectros de amplitud de Fourier de las componentes horizontal del movimiento en la superficie y del movimiento horizontal incidente en el basamento rocoso respectivamente.

Sin embargo el espectro de la componente horizontal en superficie $\mathrm{S}_{\mathrm{HS}}$ es afectado por las ondas superficiales ya que en la mayoría de los casos el ruido artificial se propaga principalmente como ondas Rayleigh. Así y de este modo SHS probablemente se encuentre "contaminado" por éstas. De esta manera, el efecto de las ondas Rayleigh debe de ser incluido en el espectro de la componente vertical del movimiento en la superficie $\mathrm{S}_{\mathrm{vs}}$ pero no en el espectro de la componente vertical del movimiento en el basamento rocoso $\mathrm{S}_{\mathrm{VB}}$.

Asumiendo que el movimiento vertical no es amplificado por las capas superficiales, la función de transferencia Es representa el efecto de las ondas Rayleigh en el movimiento vertical:

$$
E_{S}=\frac{S_{V S}}{S_{V B}}
$$

Así , si no hay ondas Rayleigh, Es= 1 e irá creciendo con el incremento de las ondas Rayleigh.

Si tenemos en cuenta que el efecto de las ondas Rayleigh es igual para las dos componentes horizontales y en la vertical en basamento, se considera que la relación

$$
S_{t t}=\frac{S_{T}}{E_{S}}
$$

es una función de transferencia más fiable, después de haber eliminado el efecto de las ondas Rayleigh obteniéndose:

$$
S_{t t}=\frac{S_{T}}{E_{S}}=\frac{R_{S}}{R_{B}}
$$

dónde:

$$
R_{S}=\frac{S_{H S}}{S_{V S}} \quad R_{B}=\frac{S_{H B}}{S_{V B}}
$$

La función Stt corresponde entonces a la función de transferencia modificada propuesta por Nakamura. $R s$ y $R b$ fueron obtenidos al dividir los espectros horizontales por los verticales en superficie (Rs) y basamento (Rb). Según mediciones realizadas por Nakamura en basamento, la 
relación $R_{b}$, es aproximadamente 1 para un amplio rango de frecuencias $(0,1$ a $20 \mathrm{~Hz})$ en sitios donde existe un subestrato firme. De este modo, la propagación es aproximadamente igual en todas las direcciones, con lo que se puede concluir que:

$$
S_{t t} \approx R_{S}
$$

Esto significa que la función de transferencia de capas superficiales puede ser estimada a partir únicamente del movimiento en superficie. Dicho de otra forma, la componente vertical en la superficie presenta las mismas características del movimiento horizontal en el basamento.

$R_{b}$ debe estar libre del efecto de ondas Rayleigh y tener características relativas al sitio por lo que una estimación más confiable de la relación Stt se produciría al multiplicarla por $1 / \mathrm{Rb}$ como término de compensación cuando se suministra la información del basamento.

Para Nakamura (1989), el método de la razón espectral permitiría obtener la función de transferencia completa del suelo, periodo y amplificación. Sin embargo algunas investigaciones y trabajos experimentales como los de Lachet y Bard (1994) han determinado que no es así, obteniéndose solo la determinación del periodo dominante.

\subsubsection{Ventajas y limitaciones del método}

Originalmente la técnica de Nakamura fue introducida para la evaluación del efecto de sitio basada en registros de microtemblores, sin embargo, esta técnica ha sido extendida a registros de movimiento débil y en algunos estudios a registros de movimiento fuerte (Lermo y ChavezGarcía, 1994).

La metodología de Nakamura ha sido objeto de numerosas investigaciones teóricas aplicadas y de simulaciones numéricas, siendo ratificada por muchos y cuestionada por otros. Sin embargo la gran mayoría ha tenido resultados con éxito, llegando a un consenso mínimo acerca de los alcances y limitaciones del método.

Así, como resultado de este consenso se puede hacer el siguiente resumen:

a) Su principal ventaja radica en la facilidad técnica y la fácil ejecución. La toma de datos y su procesamiento son muy sencillos lo que implica costos de realización muy bajos

b) Sirve para determinar los periodos fundamentales de vibración de los suelos

c) No sirve para identificar el resto de periodos fundamentales correspondientes a los siguientes modos de vibración.

d) Funciona muy bien para modelos de suelos que puede reducirse a una sola capa.

e) Los resultados de las mediciones son independientes de la hora del día no afectando a los periodos de vibración estimados. 


\subsection{Método de Tsai}

\subsubsection{Fundamento teórico}

Tsai (1969) basa su metodología en la técnica del estudio estático con el objeto de estudiar los efectos del movimiento del suelo durante la propagación de las ondas sísmicas. Su mérito se basa en extender y crear una metodología de acuerdo a este principio para un modelo con varias capas de terreno.

El modelo propuesto de capas a analizar se supone que está compuesto por un medio isotrópico, homogéneo y lineal de capas elásticas con una interfase entre ellas compuesta por una serie de planos paralelos que se extienden infinitamente en ambas direcciones (Fig. 5.1). Todo el sistema se asentará sobre un basamento de roca modelizado como un semiespacio homogéneo.

Por simplicidad el análisis se realiza solo para el caso de la incidencia de las ondas $S$ ya que estos resultados pueden ser fácilmente convertidos para el caso de ondas incidentes $P$ reemplazando la correspondiente constante elástica (al encontrarnos en régimen elástico).

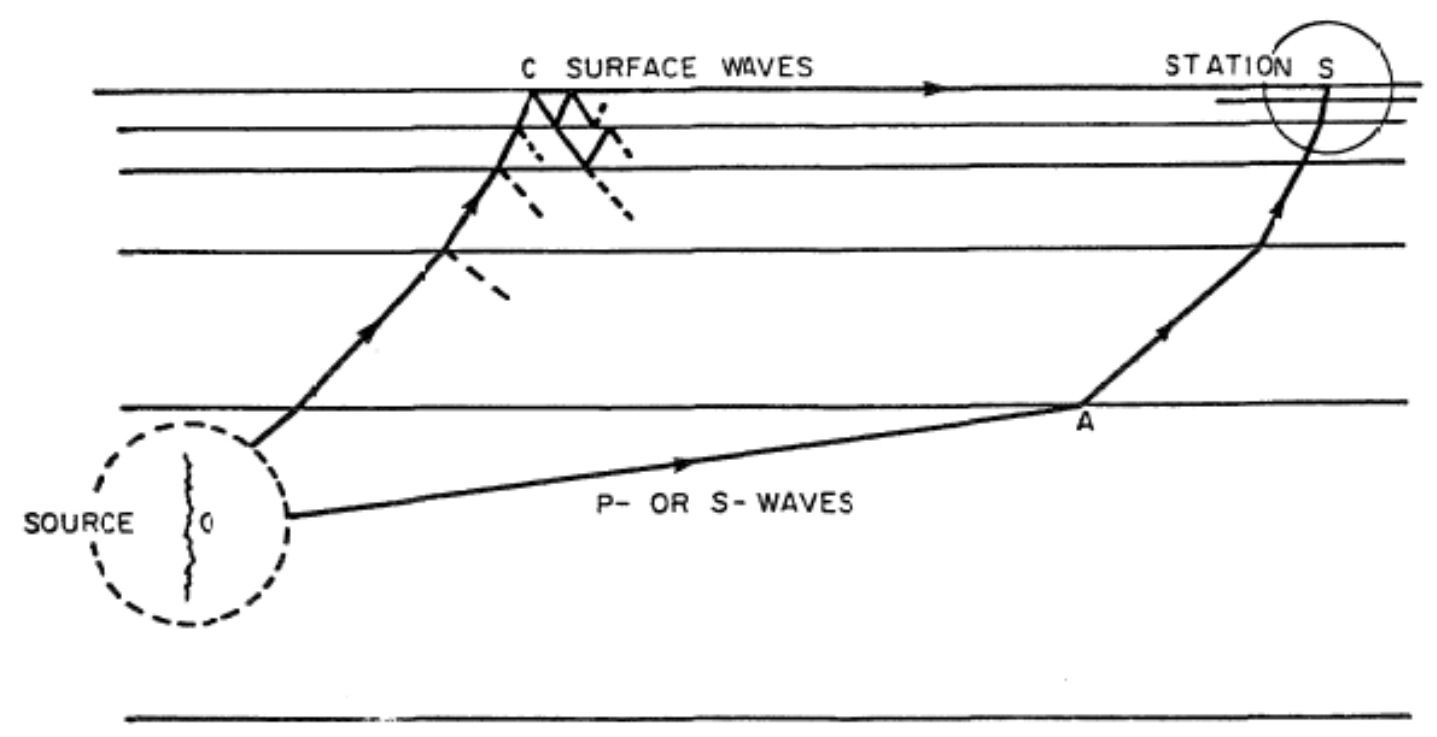

Figura5.1. Modelización del terreno en forma de capas propuesto por Tsai (1969)

La asunción de un medio con capas perfectamente elástico es solo una idealización y por esta razón, para comenzar el análisis, debemos de considerar un sistema de capas visco-elástico. Varios modelos de capas son los que comúnmente se pueden usar para este tipo de modelos; los más comunes son el modelo de Maxwell, el de Voigt-Kelvin y el estándar (Fig. 5.2). 


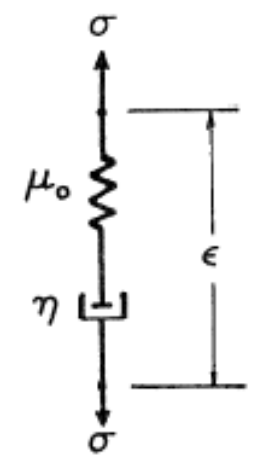

(a)

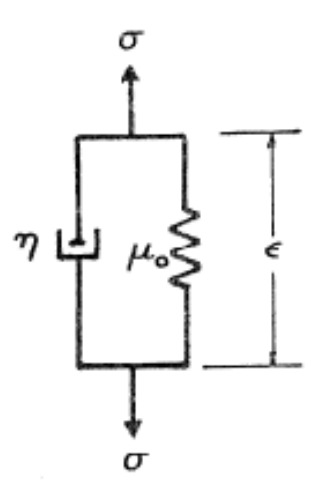

(b)

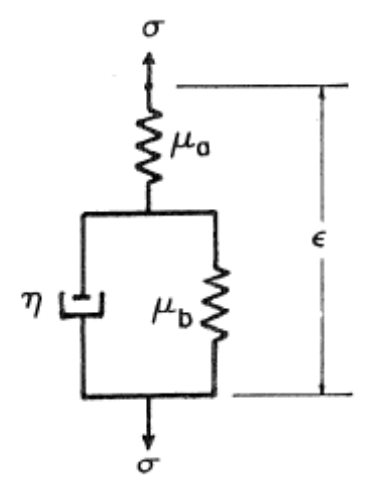

(c)

Figura 4.2. Esquema de los distintos modelos de comportamiento viscoelástico.

(a) Modelo de Maxwell (b) Modelo de Voigt-Kelvin (c) Modelo estándar

Así, la metodología de Tsai considera un modelo de $\mathrm{N}$ capas las cuáles cumplen la siguiente ley de tensiones en función de la deformación

$$
\sigma=\mu(\omega) \varepsilon=\mu_{0}\left(\frac{1+i \omega \tau}{1+i \omega A}\right) \epsilon
$$

donde:

$$
A=\frac{\tau}{1+\Gamma}
$$

El sistema de capas se presenta en la figura 4.3. dónde la capa superior se llama 1 y el basamento modelizado como semiespacio $\mathrm{N}+1$. Este conjunto de capas bajo una excitación sinusoidal tiene una respuesta que viene dada por la siguiente expresión:

$$
C_{j}^{Z}(\omega) \frac{\partial^{2} u_{j}\left(z_{j}, t\right)}{\partial z_{j}^{2}}=\frac{\partial^{2} u_{j}\left(z_{j}, t\right)}{\partial t^{2}}, \quad j=1,2, \ldots . N+1
$$

donde uj es la respuesta de la capa j. 


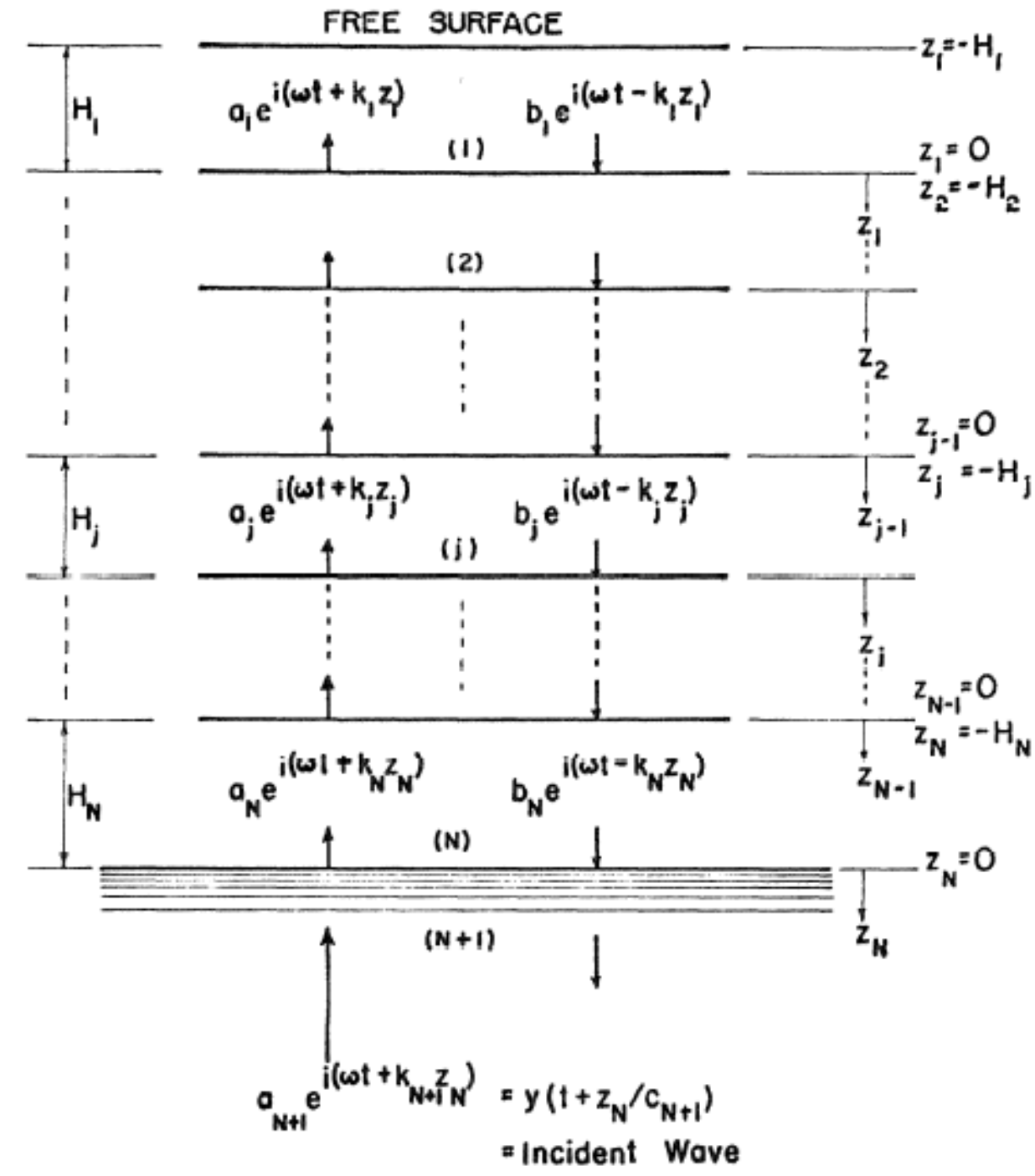

Fig. 4.3. Modelo de capas propuesto para el cálculo

La solución a este conjunto de ecuaciones diferenciales puede venir dada por la siguiente expresión:

$$
u_{j}\left(z_{j}, t\right)=a_{j} e^{i\left(\omega t+k_{j} z_{j}\right)}+b_{j} e^{i\left(\omega t-z_{j}\right)}
$$

para un zj que varía entre $-\mathrm{H}_{\mathrm{j}}$ y 0 . La determinación de los coeficientes aj y bj requiere la aplicación de una serie de condiciones de contorno para cada interfase del sistema.

De esta manera se obtiene como valores de dichos coeficientes: 


$$
\left\lceil\begin{array}{l}
a_{j} \\
b_{j}
\end{array}\right]=\frac{a_{N+1}}{\left(R e_{N+1}+R e_{N+1}^{\prime}\right)+i\left(\operatorname{Im}_{N+1}+\operatorname{Im}_{N+1}^{\prime}\right)}\left[\begin{array}{cc}
e^{i s_{j}} & 0 \\
0 & e^{-i s_{j}}
\end{array}\right]
$$

Teniendo como respuestas en la superficie del modelo de capas:

$$
u_{1}\left(-H_{1}, t\right)=\frac{2 a_{N+1}}{\sqrt{\left(R e_{N+1}+R e_{N+1}^{\prime}\right)^{2}+\left(I m_{N+1}+I m_{N+1}^{\prime}\right)^{2}}} e^{i\left(\omega t-\emptyset_{N+1}\right)}
$$

con:

$$
\emptyset_{N+1}(\omega)=\tan ^{-1}\left(\frac{I m_{N+1}+I^{\prime}{ }_{N+1}}{R e_{N+1}+R e^{\prime}}\right)
$$

siendo el angulo de fase de u1 respecto a la onda incidente cuando lleva a la base.

Se define el ratio:

$$
\operatorname{AMP}(\omega)=\frac{\left|u_{1}\left(-H_{1}, t\right)\right|}{|2 y(t)|}=\frac{\left|u_{1}\left(-H_{1}, t\right)\right|}{\left|2 a_{2 N+1}\right|}
$$

Este nos dará una relación entre el movimiento entre la capa de basamento y la capa superficial, siendo fundamental a la hora de tener la función de transferencia entre ambos que nos permita obtener la amplificación en superficie. 


\section{6.- fp Y AMPLIFICACIÓN OCON LOS MÉTODOS DE DE NAKAMURA Y TSAI}

\section{1.- Resultados del Método de Nakamura}

Al aplicar el Método de Nakamura a los datos de la estación central ( de tres componentes) se han obtenido los periodos predominantes del sitio. En las figura 6.1 a 6.12 se muestran las razones $\mathrm{H} / \mathrm{V}$ obtenidas en cada estación, donde también se muestran las frecuencias predominantes del sitio. El punto señalado con un recuadro indica la frecuencia que concuerda con lo obtenido con el método de Tsai. A continuación se presentan los gráficos correspondientes a los resultados obtenidos con dicho método en todos los puntos de estudio analizados.

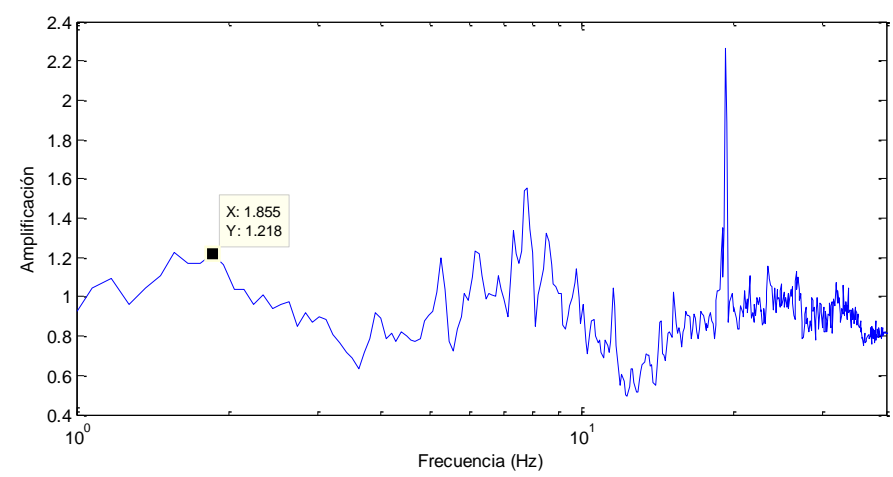

Figura 6.1. Razón H/V obtenida en la estación de Aynadamar donde se muestran las frecuencias predominantes del sitio. El punto señalado con un recuadro indica la frecuencia en con la que se obtiene el máximo en el método de Tsai.

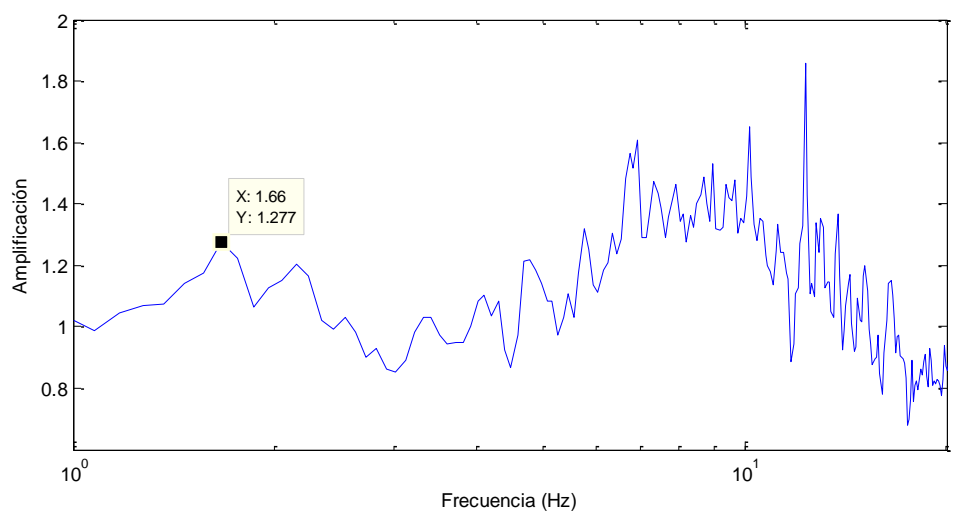

Figura 6.2. Razón H/V obtenida en la estación de Beiro donde se muestran las frecuencias predominantes del sitio. 


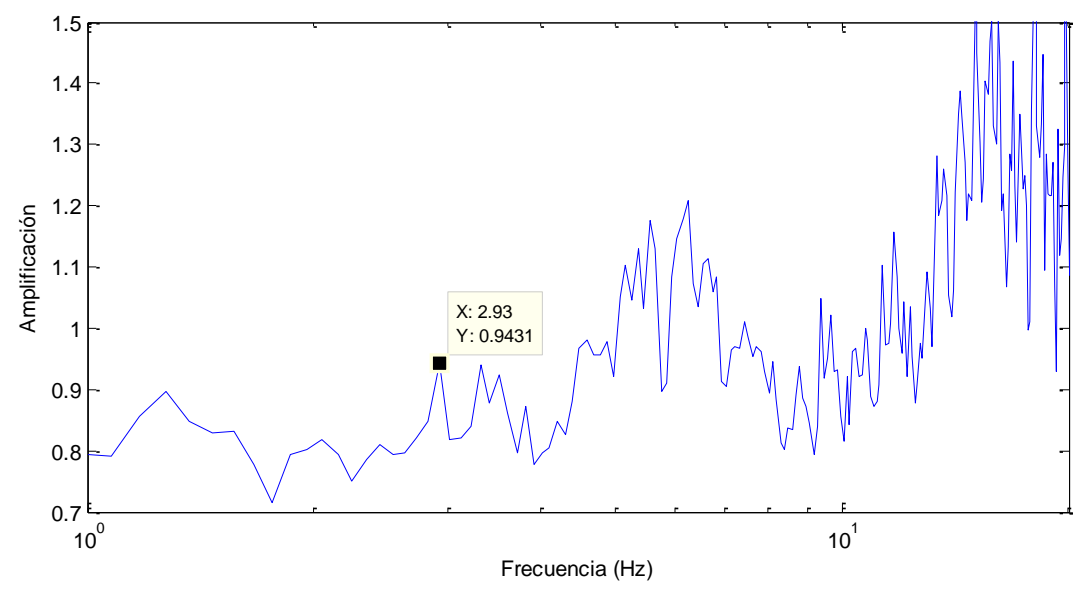

Figura 6.3. Razón H/V obtenida en la estación de Bola de Oro donde se muestran las frecuencias predominantes del sitio.

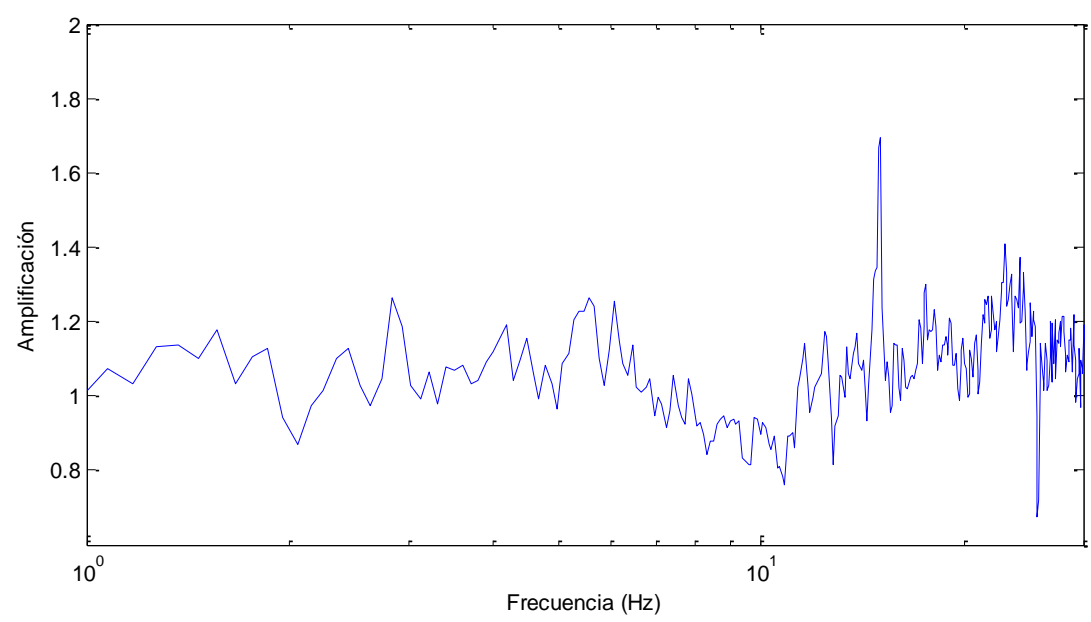

Figura 6.4. Razón H/V obtenida en la estación de Chana donde se muestran las frecuencias predominantes del sitio.

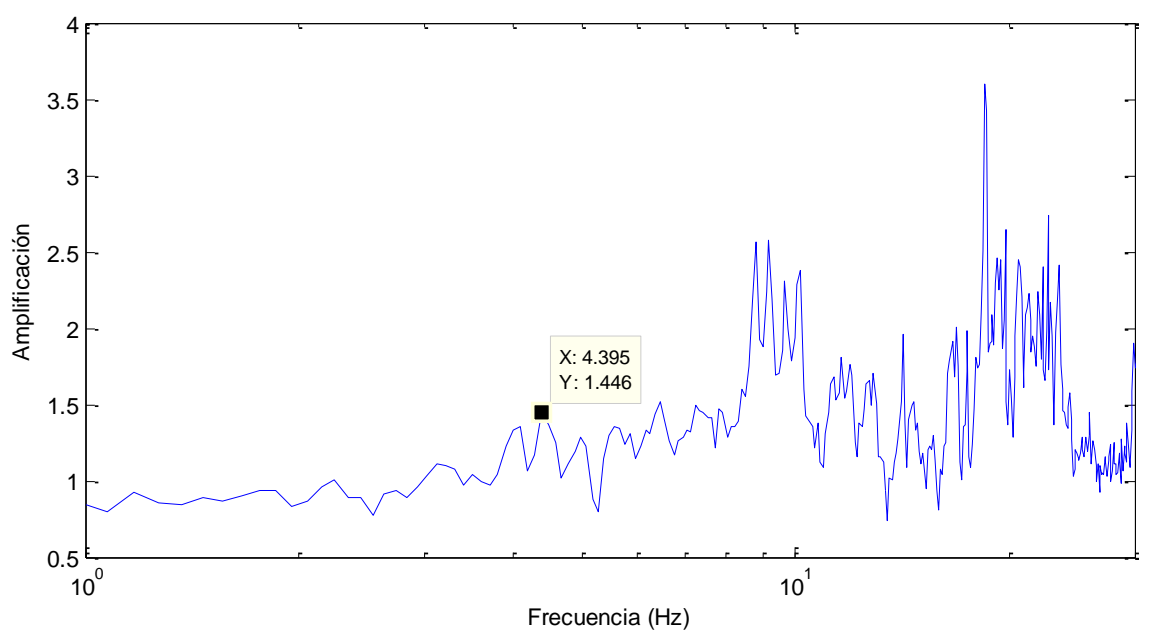

Figura 6.5. Razón H/V obtenida en la estación de Estadio de la Juventud donde se muestran las frecuencias predominantes del sitio. 


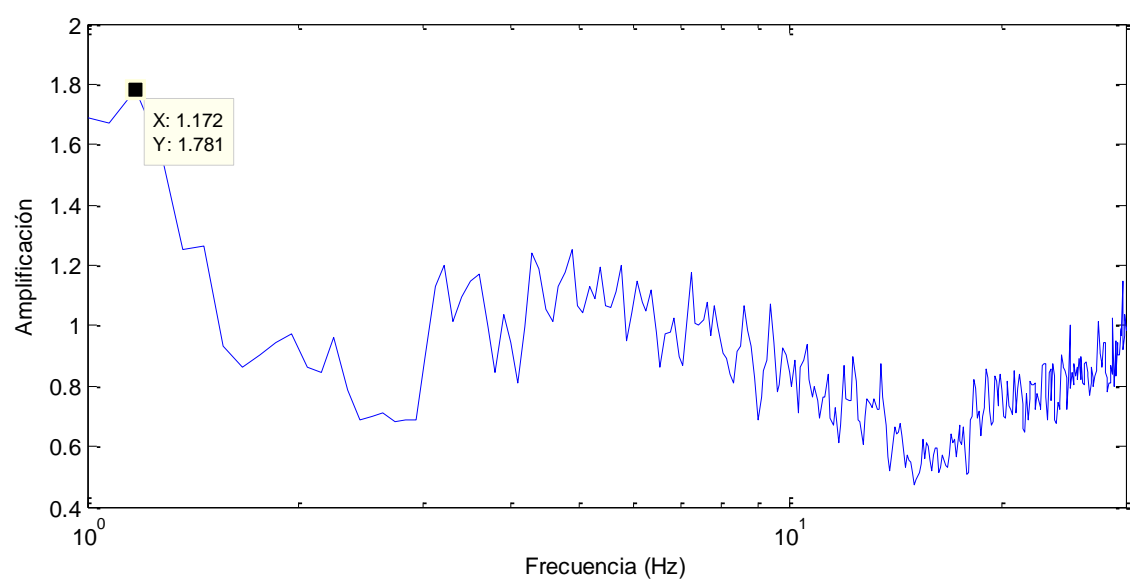

Figura 6.6. Razón $\mathrm{H} / \mathrm{V}$ obtenida en la estación de Ferial donde se muestran las frecuencias predominantes del sitio.

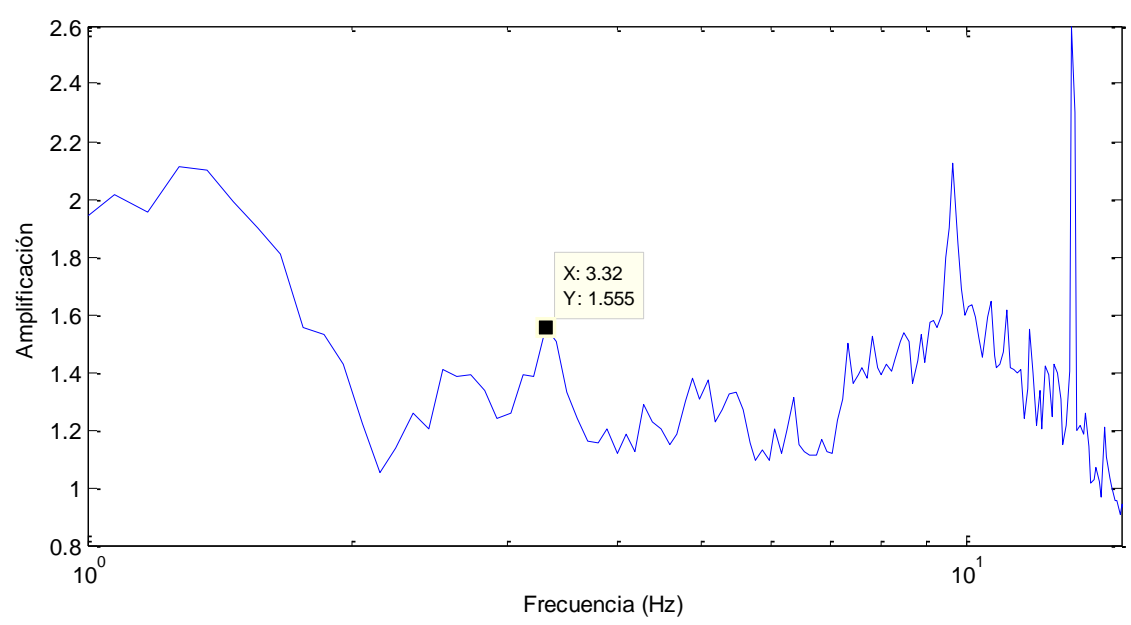

Figura 6.7. Razón $\mathrm{H} / \mathrm{V}$ obtenida en la estación de Fuentenueva donde se muestran las frecuencias predominantes del sitio.

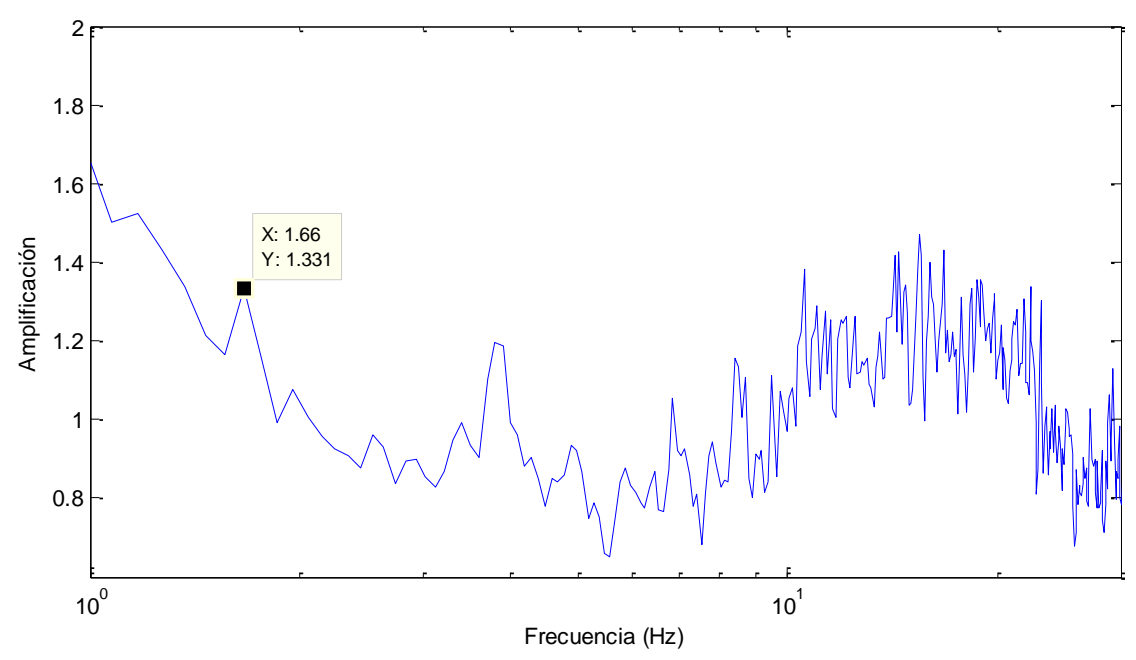

Figura 6.8. Razón H/V obtenida en la estación de Granada $\mathbf{7 4}$ donde se muestran las frecuencias predominantes del sitio. 


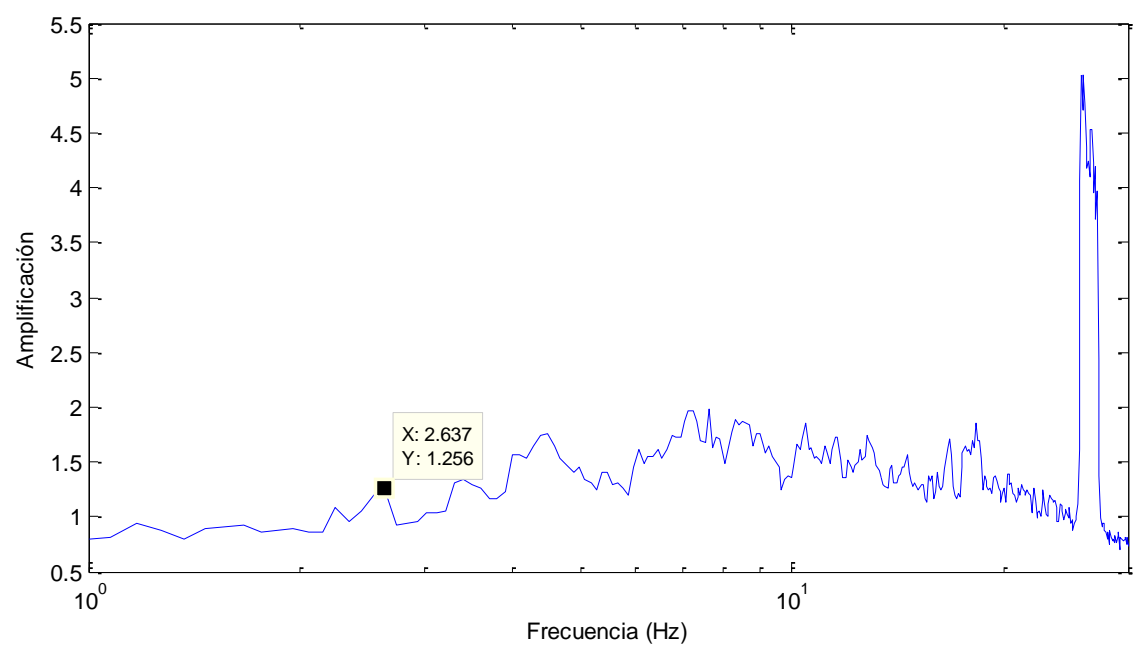

Figura 6.9. Razón H/V obtenida en la estación de Náyades donde se muestran las frecuencias predominantes del sitio.

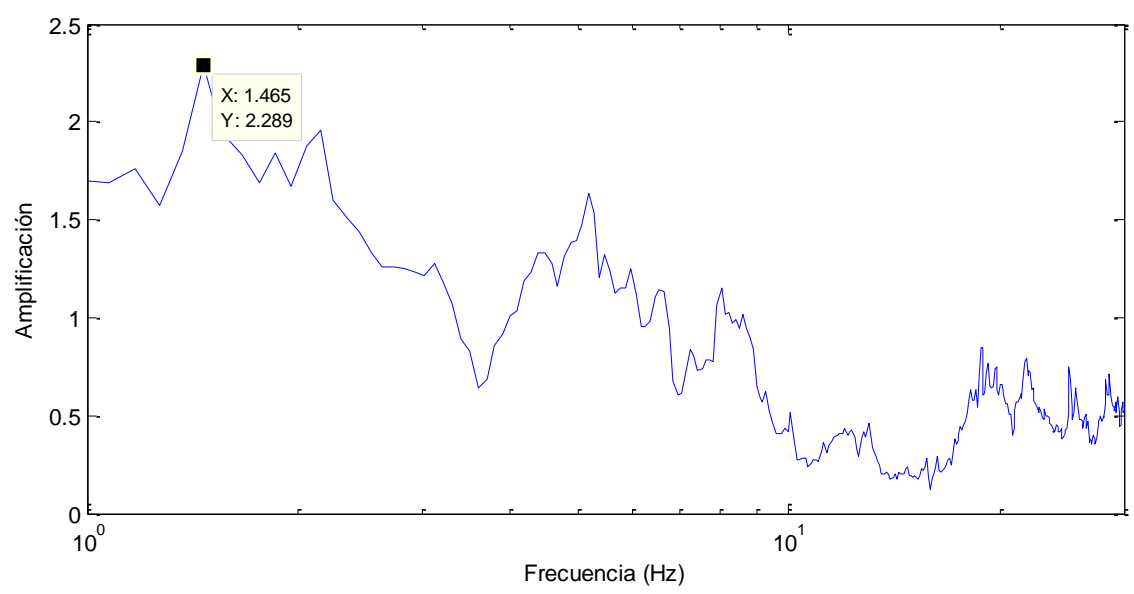

Figura 6.10. Razón H/V obtenida en la estación de Sanders donde se muestran las frecuencias predominantes del sitio.

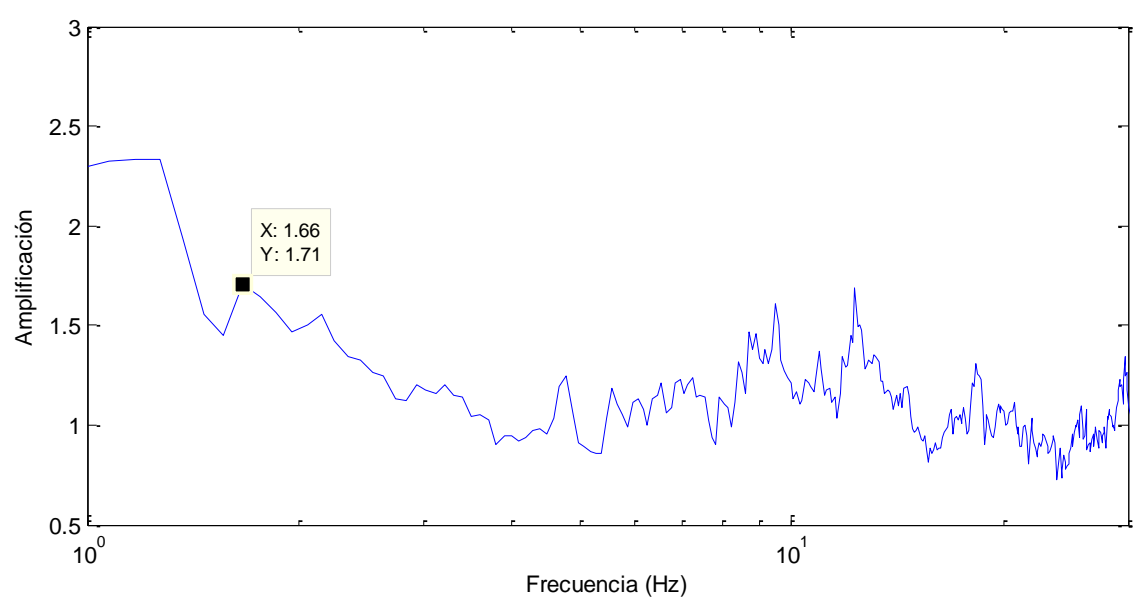

Figura 6.11. Razón H/V obtenida en la estación de Vega donde se muestran las frecuencias predominantes del sitio. 


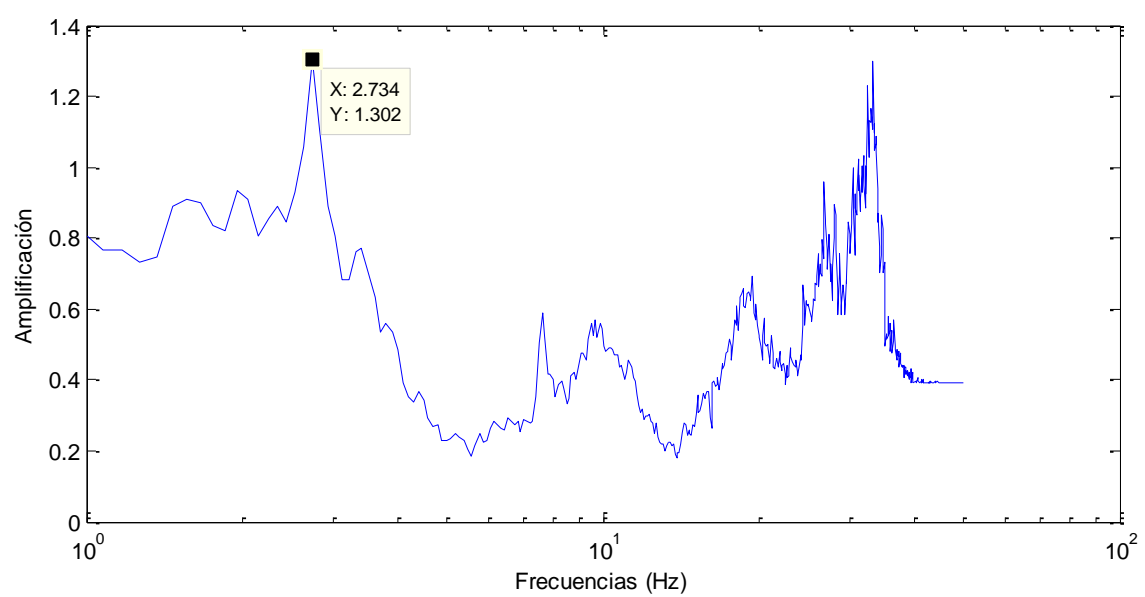

Figura 6.12. Razón $\mathrm{H} / \mathrm{V}$ obtenida en la estación de Zaidín donde se muestran las frecuencias predominantes del sitio.

\section{2.- Resultados con la técnica de Tsai}

Al aplicar el Método de Tsai con los datos de las estructura Vs obtenidas de los SPAC, hemos obtenido la función de transferencia del terreno en cada uno de los lugares. Para obtener los resultados se han utilizado uno código MatLab, implementado especialmente para este trabajo. Es de destacar que el factor de amortiguamiento utilizado para el cálculo del método de Tsai (1969) es del 5\%. Este valor ha sido estimado a partir de otros valores y de varios estudios de Ordaz et al., ya que no existen datos medidos en los puntos en estudio.

En las figura 6.13 a 6.26 se muestran las funciones de transferencia del terreno en cada sitio. En ellas también se muestra el máximo obtenido con este método en cada uno delos puntos de estudio analizados.

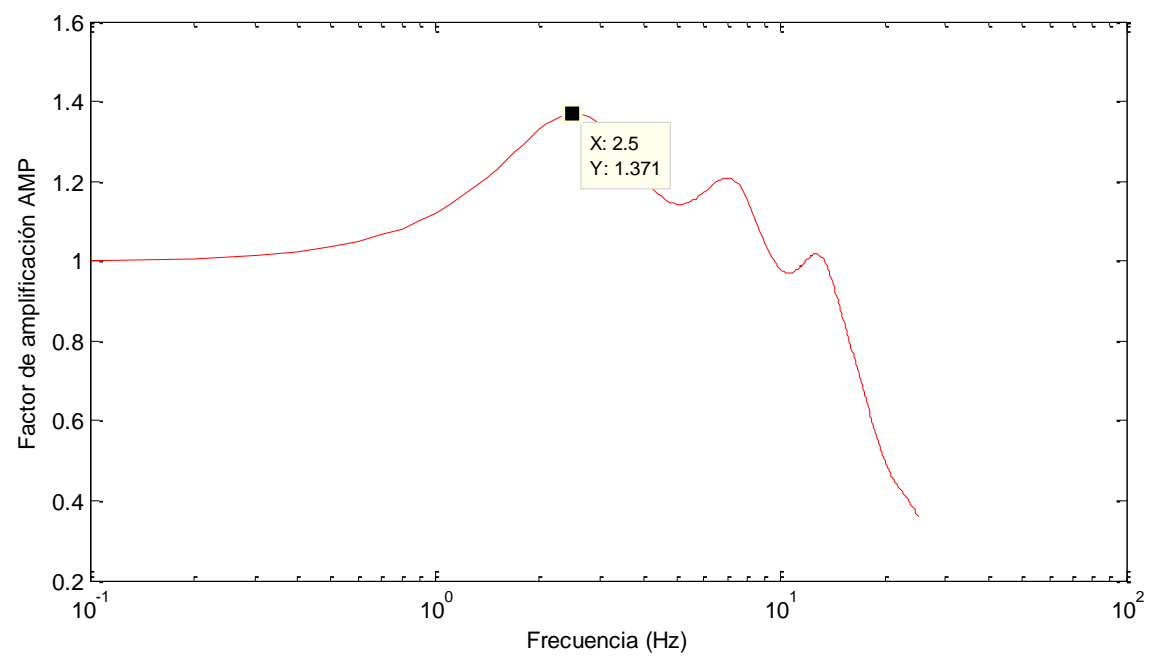

Figura 6.13. Función de transferencia del sitio en el lugar de Aynadamar. El punto señalado con un recuadro indica la frecuencia que es similar a la del método de Nakamura. 


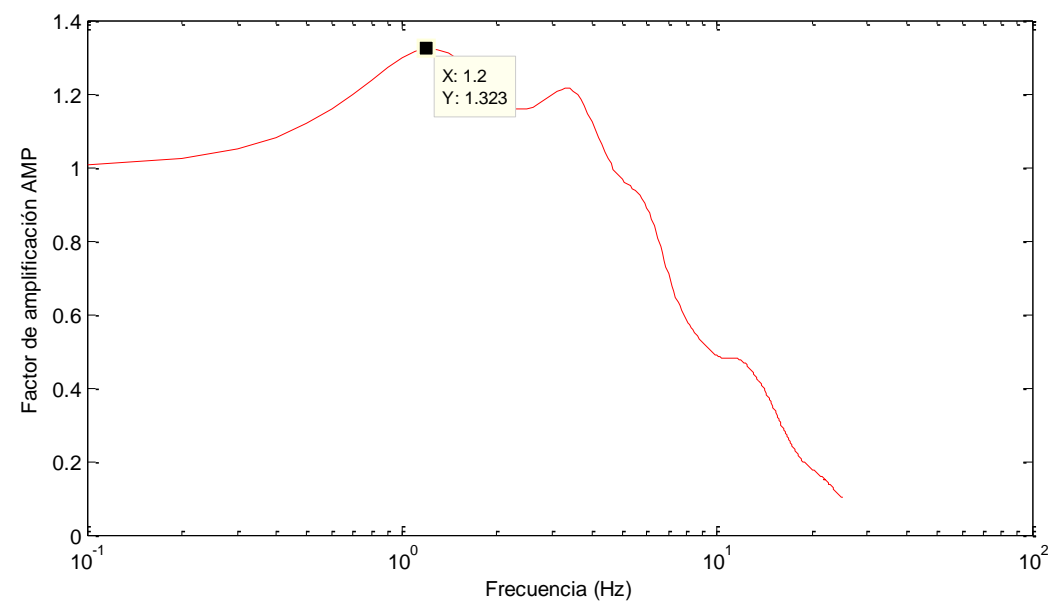

Figura 6.14. Función de transferencia del sitio en el lugar de Beiro. El punto señalado con un recuadro indica la frecuencia que es similar a la del método de Nakamura.

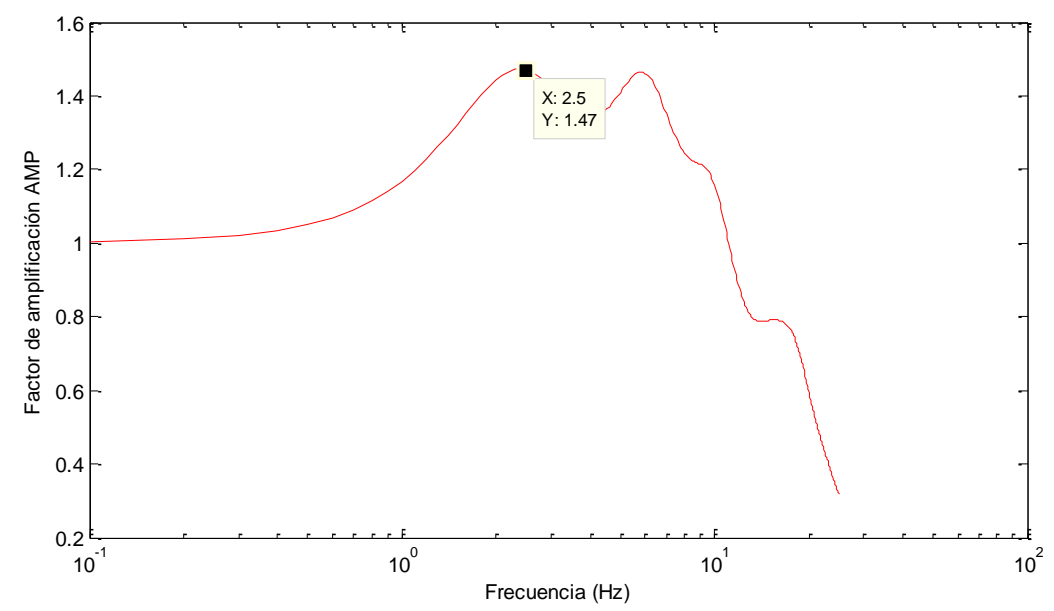

Figura 6.15. Función de transferencia del sitio en el lugar de Bola de Oro . El punto señalado con un recuadro indica la frecuencia que es similar a la del método de Nakamura.

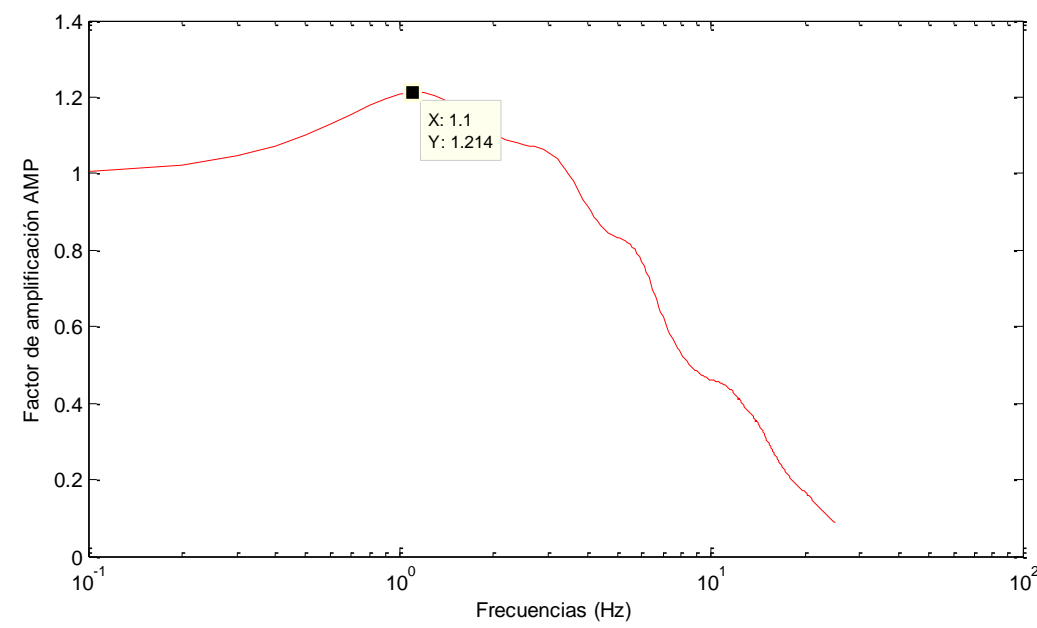

Figura 6.16. Función de transferencia del sitio en el lugar de Chana . El punto señalado con un recuadro indica la frecuencia que es similar a la del método de Nakamura. 


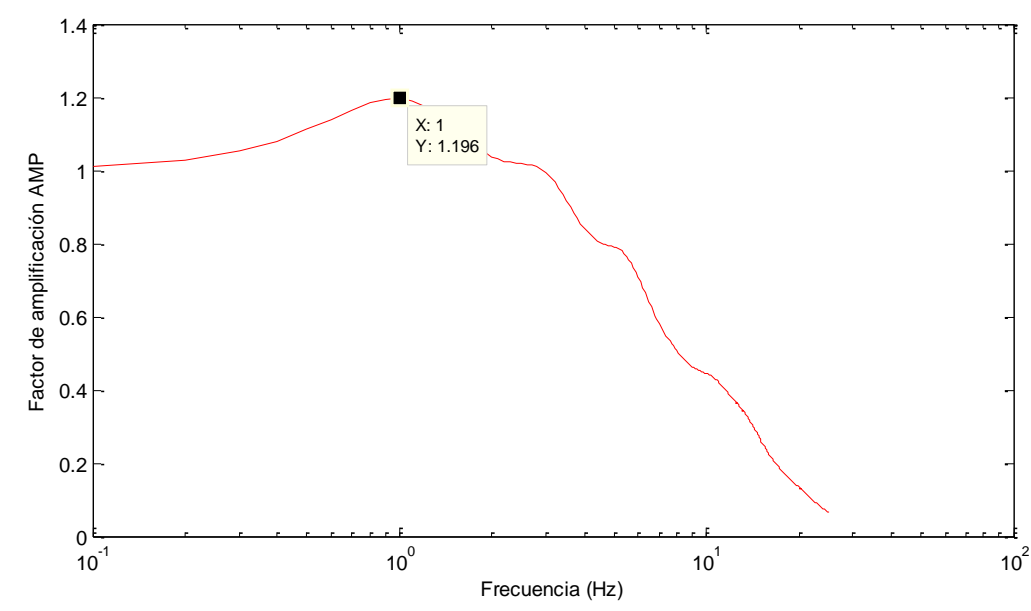

Figura 6.17. Función de transferencia del sitio en el lugar de Estadio . El punto señalado con un recuadro indica la frecuencia que es similar a la del método de Nakamura.

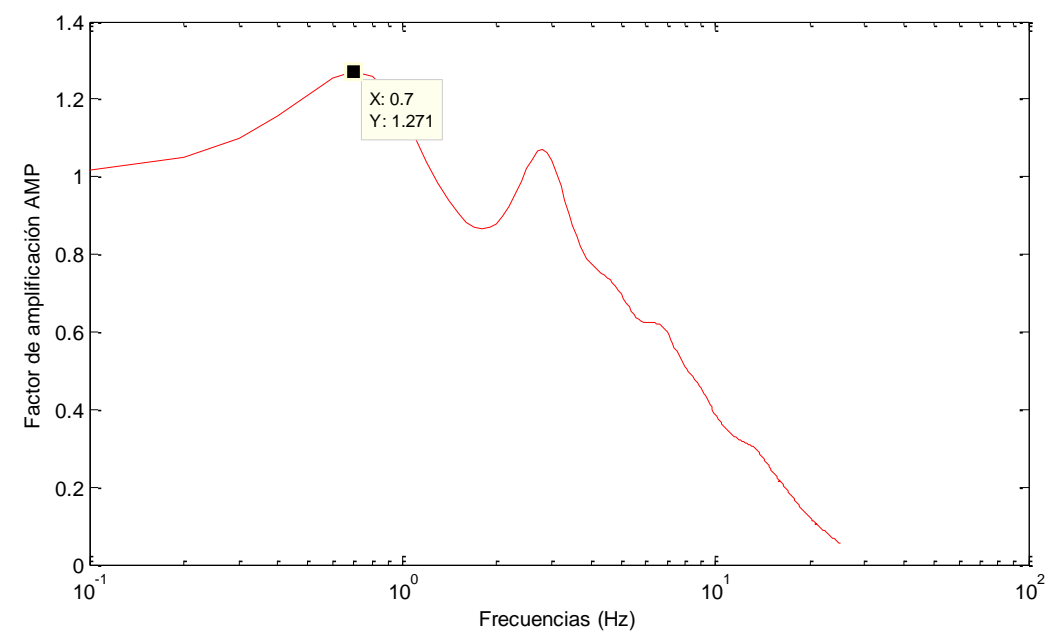

Figura 6.18. Función de transferencia del sitio en el lugar de Ferial. El punto señalado con un recuadro indica la frecuencia que es similar a la del método de Nakamura.

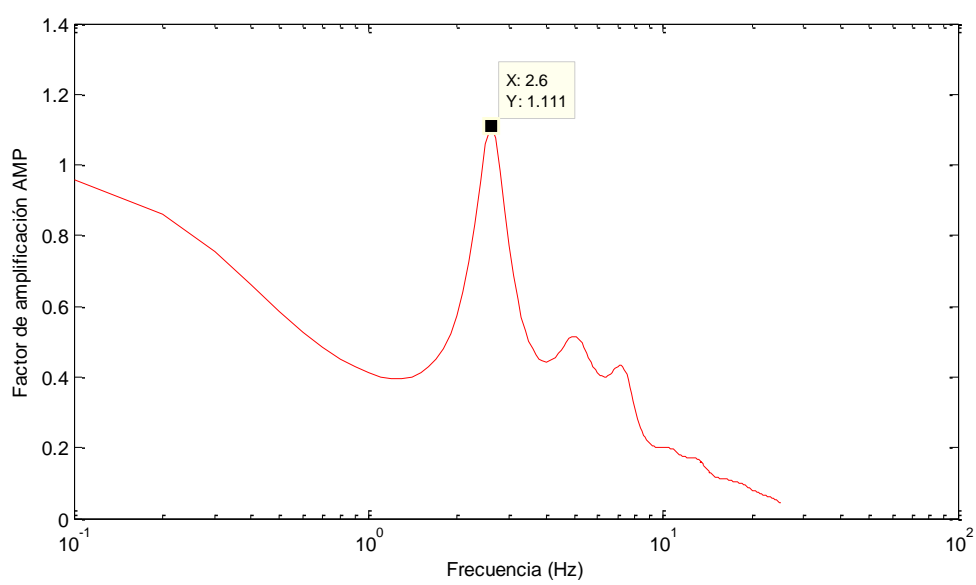

Figura 6.19. Función de transferencia del sitio en el lugar de Fuentenueva 1. El punto señalado con un recuadro indica la frecuencia que es similar a la del método de Nakamura. 


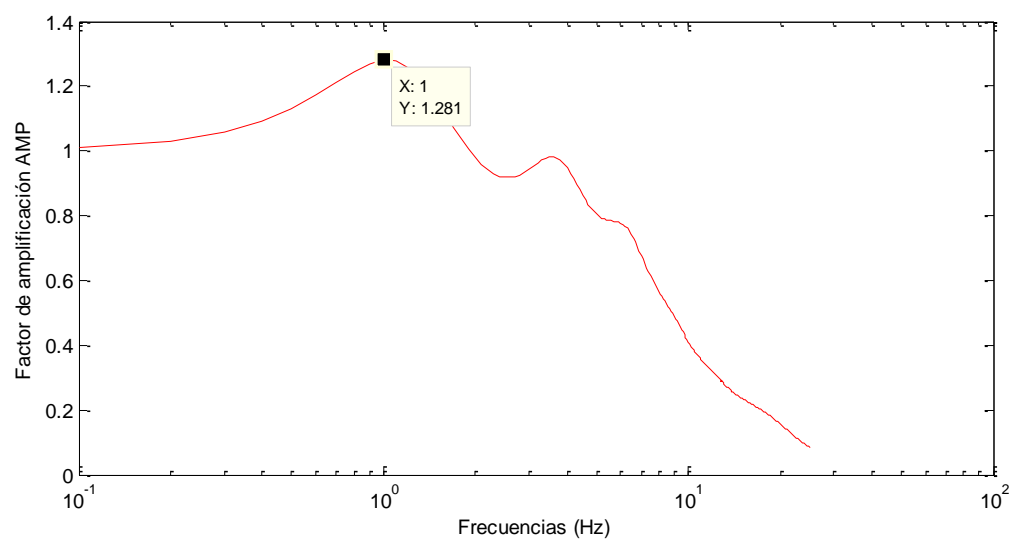

Figura 6.20. Función de transferencia del sitio en el lugar de Fuentenueva 2. El punto señalado con un recuadro indica la frecuencia que es similar a la del método de Nakamura.

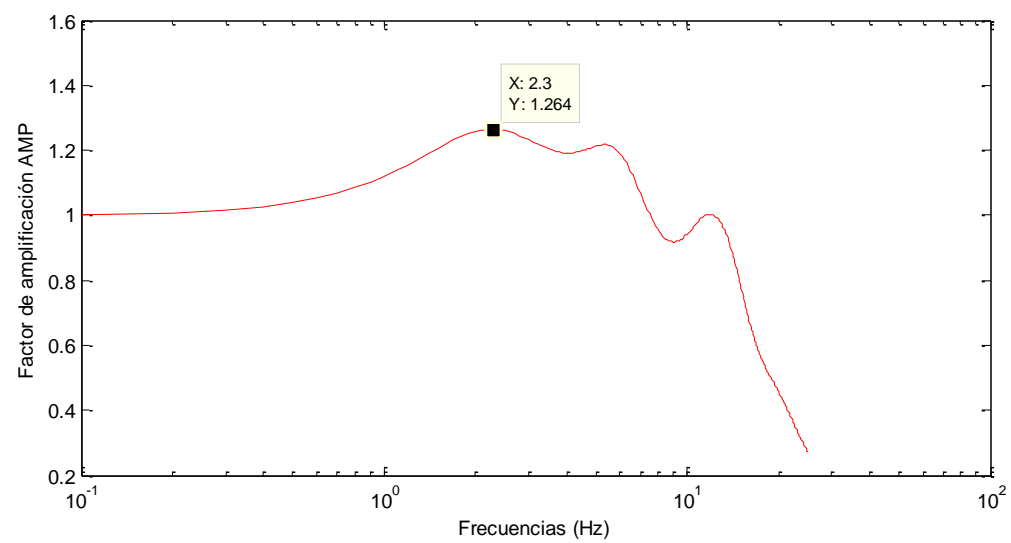

Figura 6.21. Función de transferencia del sitio en el lugar de Granada 74. El punto señalado con un recuadro indica la frecuencia que es similar a la del método de Nakamura.

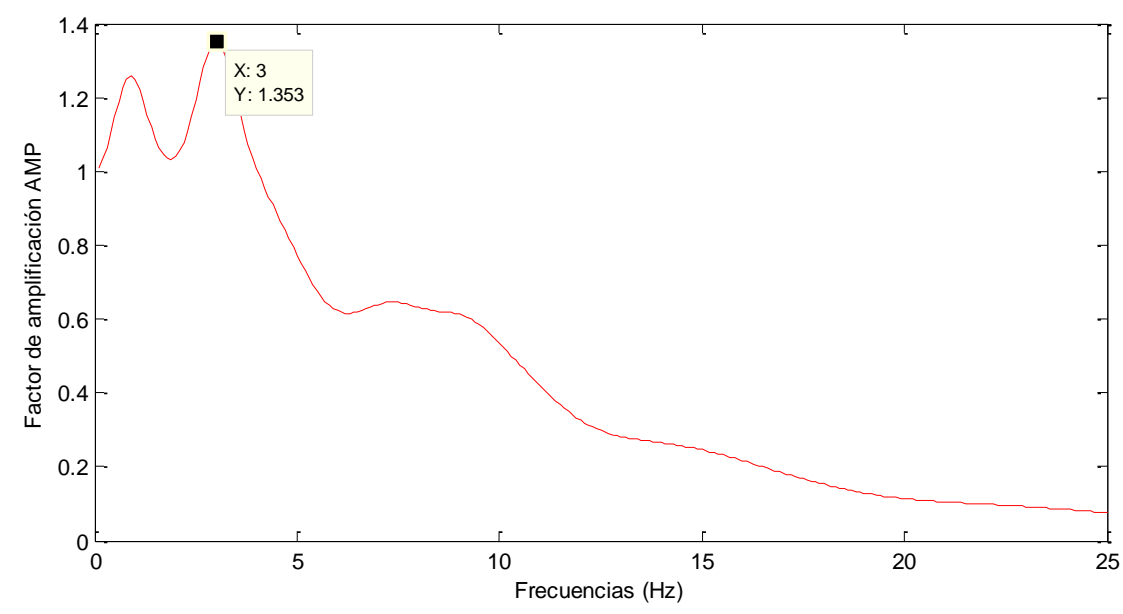

Figura 6.22. Función de transferencia del sitio en el lugar de Náyades. El punto señalado con un recuadro indica la frecuencia que es similar a la del método de Nakamura. 


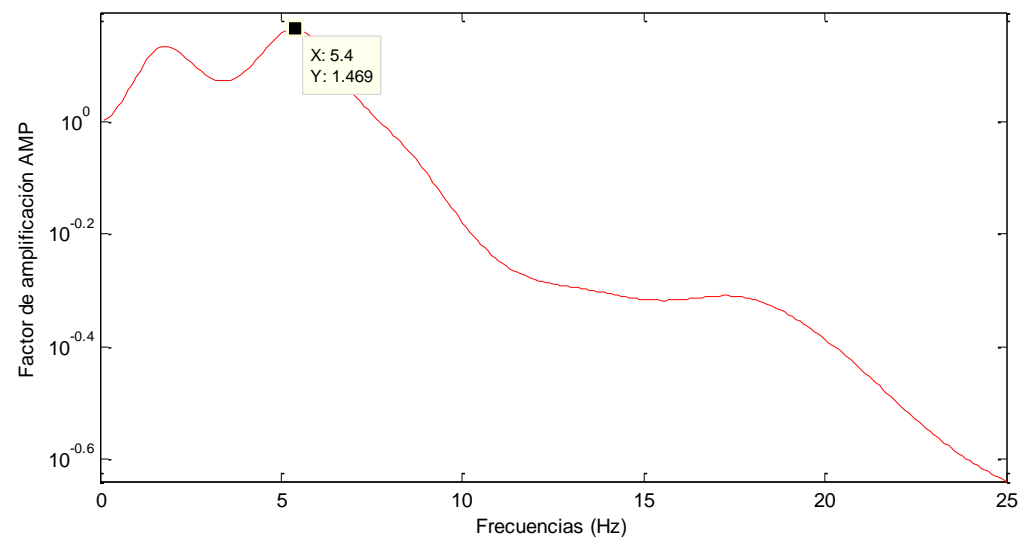

Figura 6.23. Función de transferencia del sitio en el lugar de Parque de las ciencias . El punto señalado con un recuadro indica la frecuencia que es similar a la del método de Nakamura.

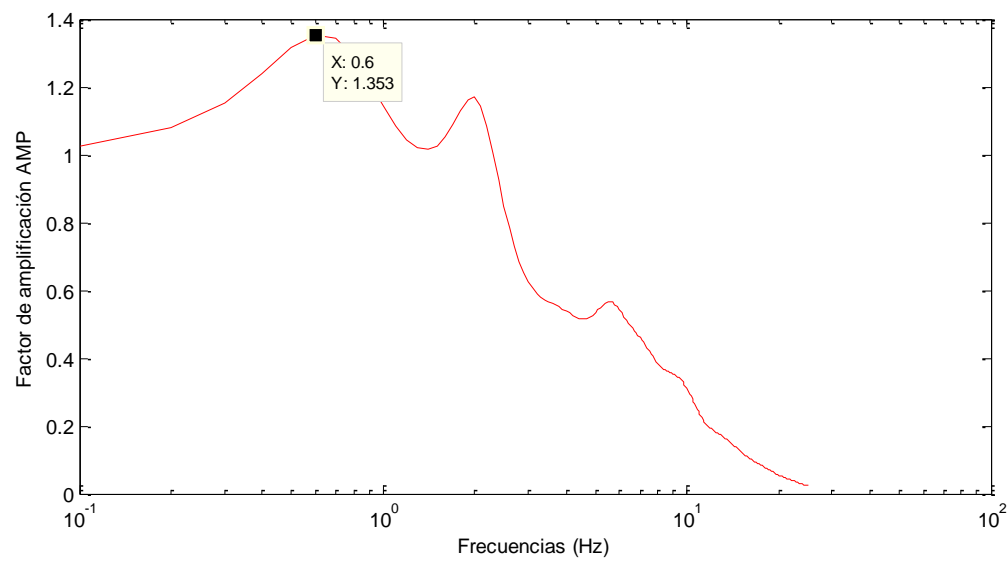

Figura 6.24. Función de transferencia del sitio en el lugar de Sanders. El punto señalado con un recuadro indica la frecuencia que es similar a la del método de Nakamura.

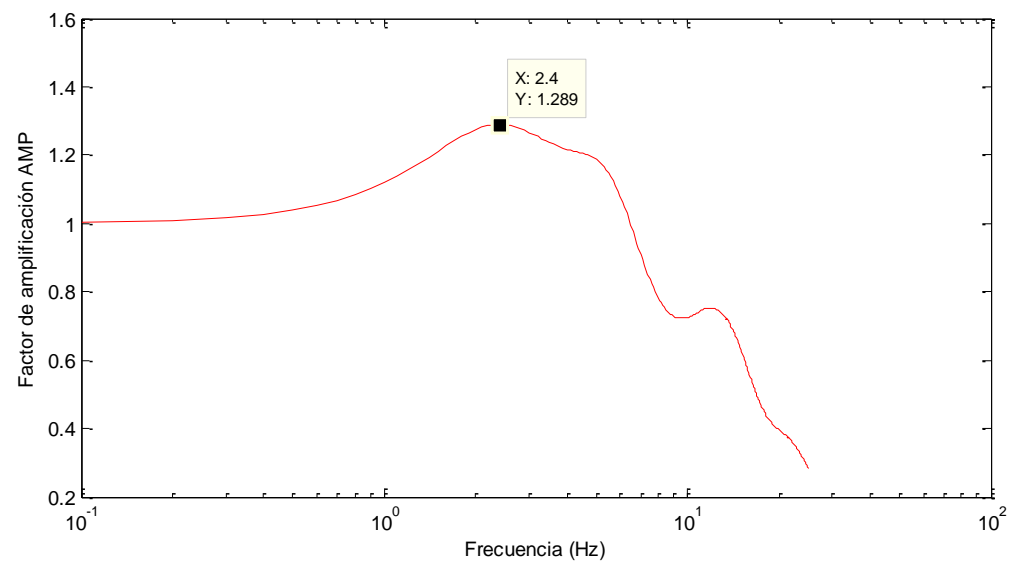

Figura 6.25. Función de transferencia del sitio en el lugar Vega. El punto señalado con un recuadro indica la frecuencia que es similar a la del método de Nakamura. 


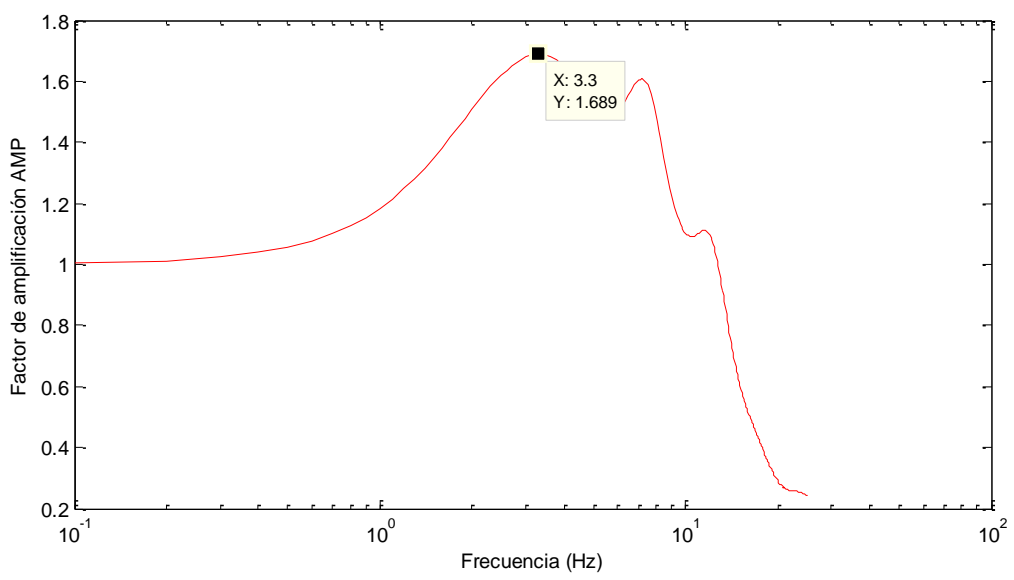

Figura 6.26. Función de transferencia del sitio en el lugar de Zaidín. El punto señalado con un recuadro indica la frecuencia que es similar a la del método de Nakamura.

Para tener una visión de las amplificaciones obtenidas con la técnica de Tsai, en la figura 6.27 se han indicado en diferentes colores los factores de amplificación. Los valores máximos se han encontrado en sitios de suelos von menor $V_{S}^{30}$ o en rellenos antrópicos. Hay que señalar que estos factores son dependientes del amortiguamiento utilizado.

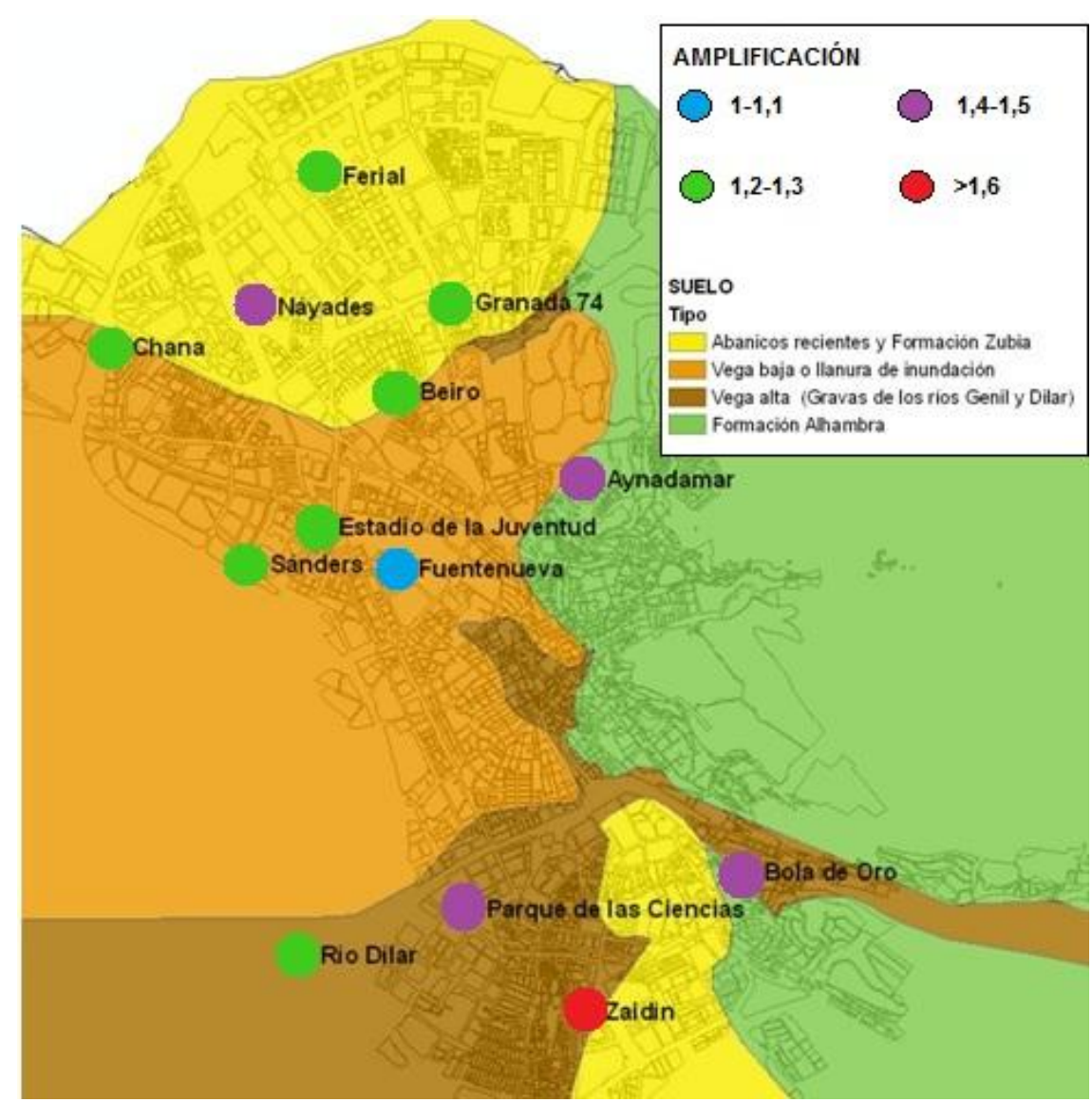

Figura 6.27. Mapa resumen de las amplificaciones obtenidas para los distintos puntos. 


\subsection{Comparativa de resultados}

Como se indicó anteriormente

Tabla 6.1 Cuadro resumen de resultados para ambos métodos

\begin{tabular}{|c|c|c|c|c|}
\hline \multirow{2}{*}{ Estación } & \multicolumn{2}{|c|}{ Método de Tsai } & \multicolumn{2}{c|}{ Método de Nakamura (razón H/V) } \\
\cline { 2 - 5 } & Amplific. máx. & Frecuencia & $\begin{array}{c}\text { Amplif. max. en } \\
\text { la frecuencia de } \\
\text { Tsai }\end{array}$ & $\begin{array}{c}\text { Frecuencia } \\
\text { predominante }\end{array}$ \\
\hline Aynadamar & 1,4 & 2,5 & 1,21 & 1,8 \\
\hline Beiro & 1,3 & 1,2 & 1,277 & 1,66 \\
\hline Bola de Oro & 1,5 & 2,5 & 1,25 & 6,2 \\
\hline Chana & 1,2 & 1,1 & 1,3 & 2 \\
\hline $\begin{array}{c}\text { Estadio de la } \\
\text { juventud }\end{array}$ & 1,2 & 1 & 1,44 & 4,39 \\
\hline Ferial & 1,2 & 0,7 & 1,78 & 1,17 \\
\hline Fuentenueva & 1,1 & 2,6 & 1,55 & 3,32 \\
\hline Granada 74 & 1,3 & 2,3 & 1,33 & 1,66 \\
\hline Náyades & 1,4 & 3 & 1,8 & 4,5 \\
\hline $\begin{array}{c}\text { Parque de las } \\
\text { Ciencias }\end{array}$ & 1,5 & 5,4 & & \\
\hline Sanders & 1,3 & 0,6 & 1,465 & 2,289 \\
\hline Vega & 1,3 & 2,4 & 1,71 & 1,66 \\
\hline Zaidín & 1,7 & 3,3 & 1,302 & 2,734 \\
\hline
\end{tabular}




\section{OBTENCIÓN DE LOS INPUT DE ENERGÍA EN SUELO USANDO DOS TERREMOTOS TIPO}

\section{1 Aplicación a dos terremotos tipo}

En este apartado se va a realizar la aplicación de algunas de las funciones de transferencia obtenidas para los diferentes puntos en estudio a unos terremotos seleccionados especialmente por ser sus características similares a los que se producen en la ciudad de Granada.

Hay que añadir que los terremotos seleccionados se tratan de terremotos en basamento rocoso por lo que sus registros no están "contaminados" por transferencia a través de terrenos de relleno. Así dicha aplicación va a ir encaminada a observar las diferencias entre los input de espectros de energía en el basamento y en superficie. Los sismos elegidos son los recientes de Valnerina y Basso Tirreno (Italia). Los registros de estos sismos se han tomado de la Base Europea de Movimientos Fuertes.

Los espectros de energía se han representado en el dominio de la frecuencia y no en el del tiempo porque las funciones de transferencia calculadas para hallar el efecto de sitio están en el dominio de la frecuencia y por tanto resultaba más fácil de visualizar su influencia.

\subsection{Terremoto de "Valnerina" (Italia)}

Las características del registro de este sismo se recogen en el Apéndice 2. Para obtener el espectro de input de energía en el basamento del movimiento horizontal se ha usado el espectro de input de energía (del promedio de las dos componentes horizontales) obtenido en tres estaciones situadas en roca.

Los resultados obtenidos en cada una de las estaciones se presentan en las figuras 7.1 a 7.13. En ellas se han representado los espectros de input de energía en el basamento y en el suelo y se reflejan en distintos colores los correspondientes a cada una de las tres estaciones. 


\section{Aynadamar}

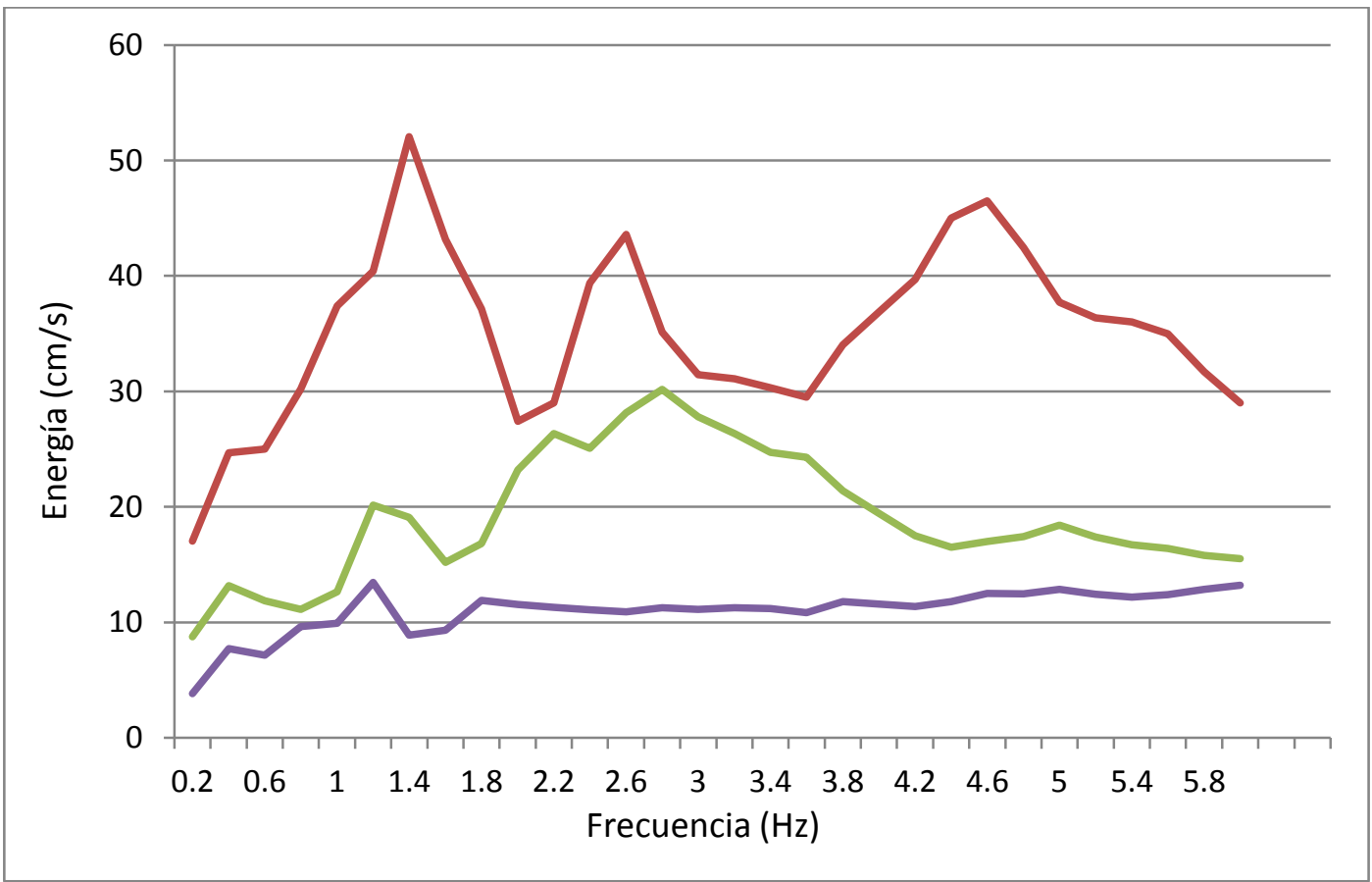

Figura 7.1a. Espectros de input de energía obtenidos antes de aplicar la función de amplificación

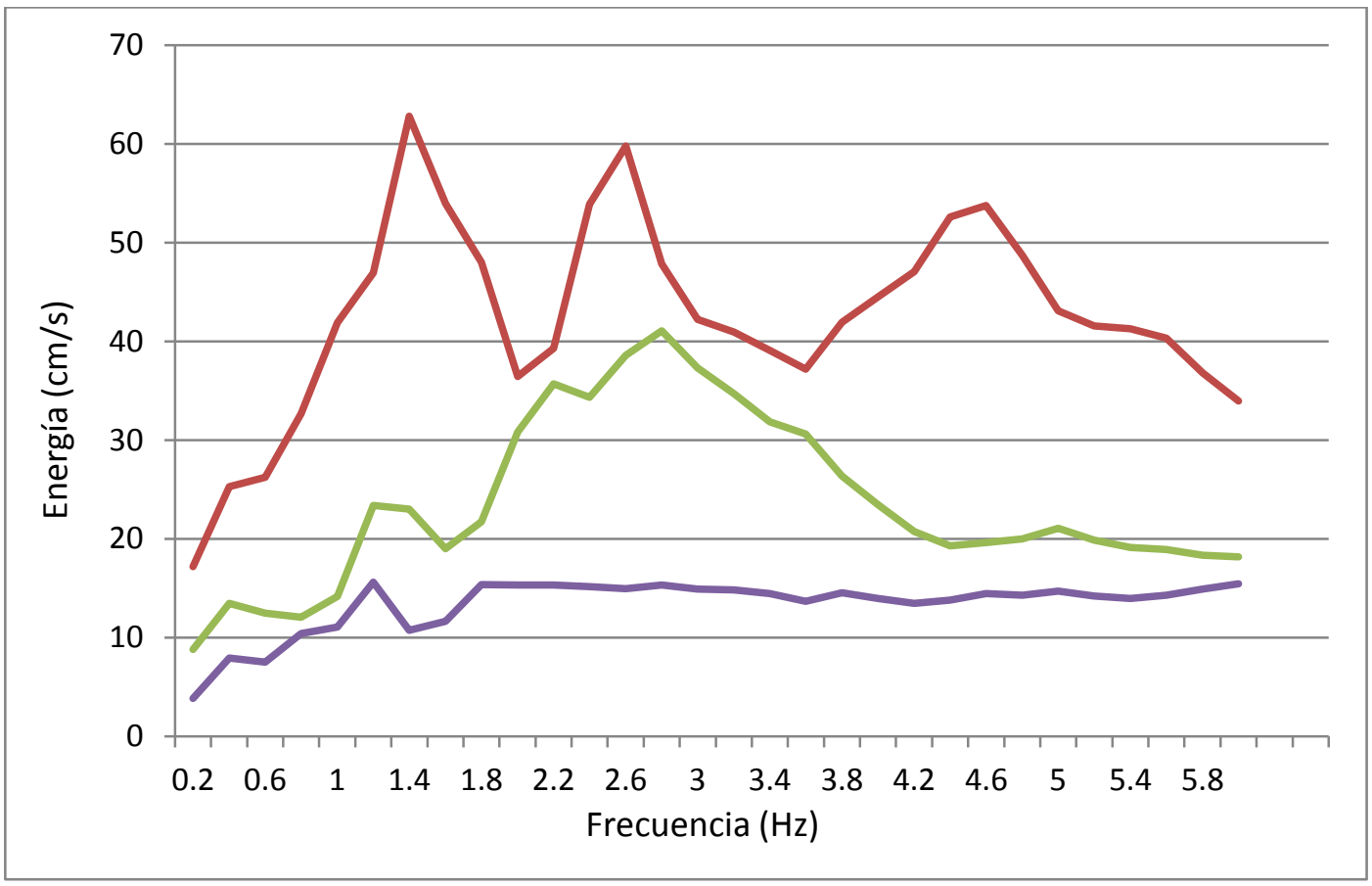

Figura 7.1b. Espectros de input de energía obtenidos tras aplicar la función de amplificación 
Beiro

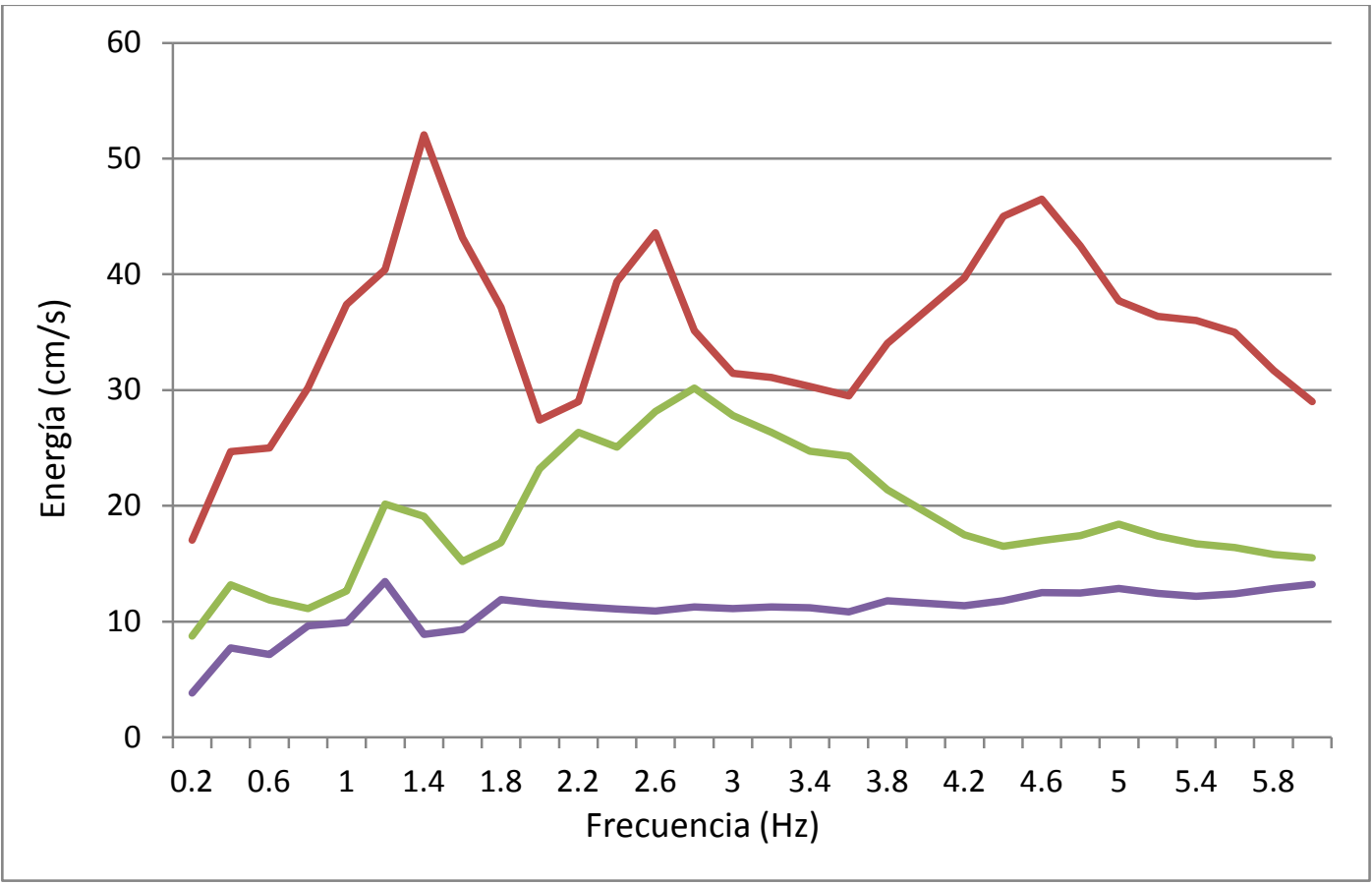

Figura 7.2a. Espectros de input de energía obtenidos antes de aplicar la función de amplificación

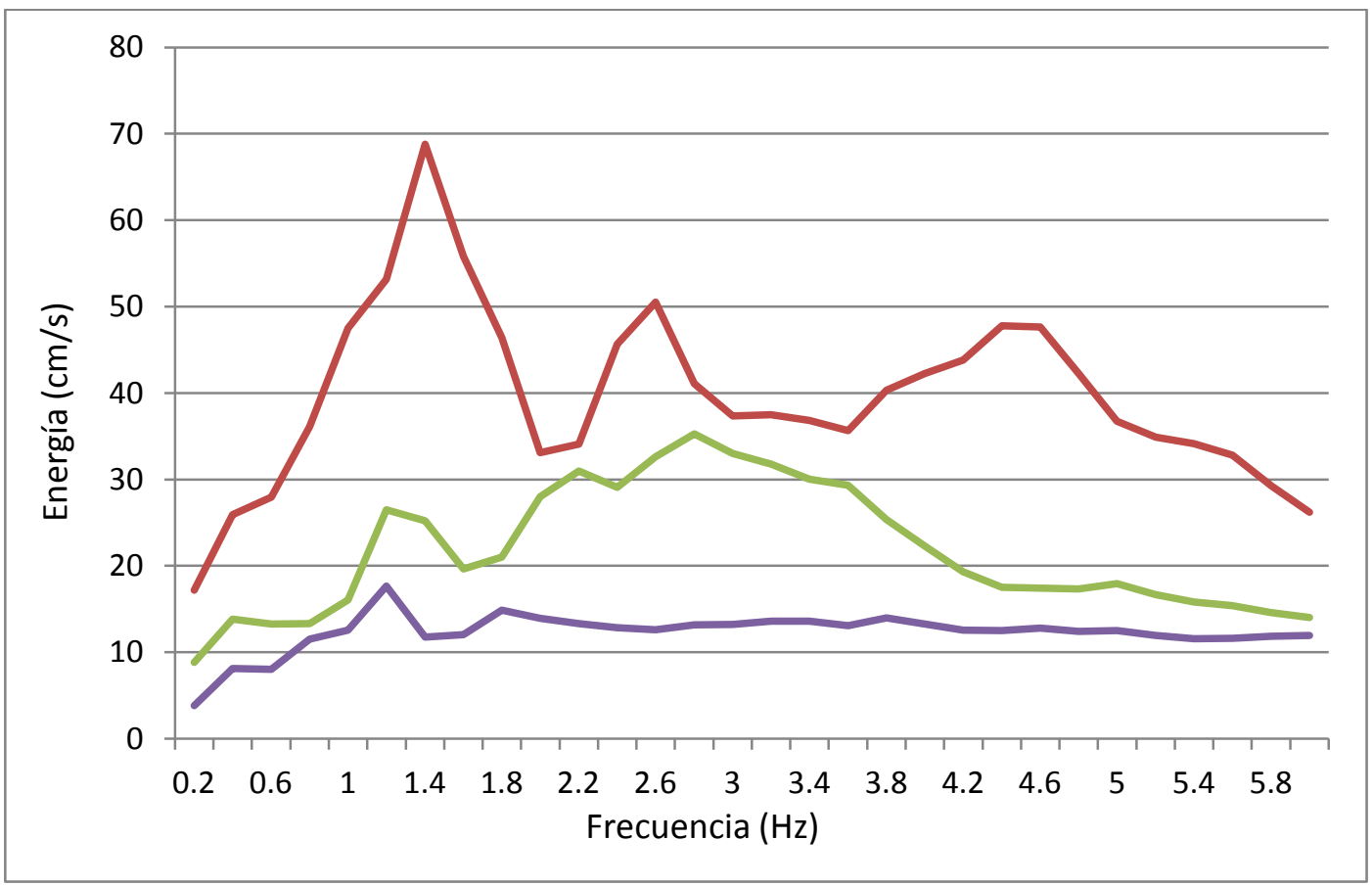

Figura 7.2b. Espectros de input de energía obtenidos tras aplicar la función de amplificación 
Bola de Oro

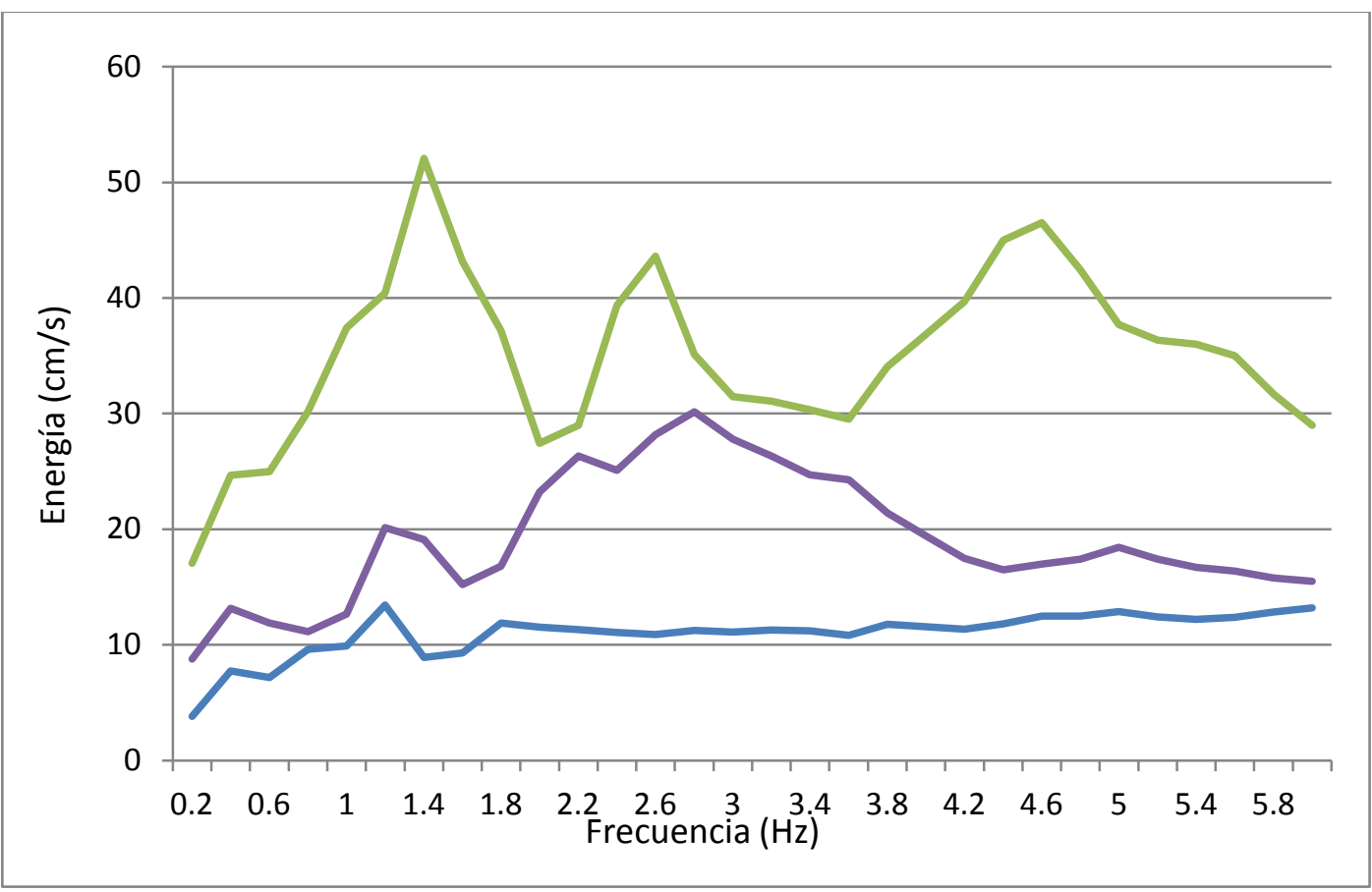

Figura 7.3a. Espectros de input de energía obtenidos antes de aplicar la función de amplificación

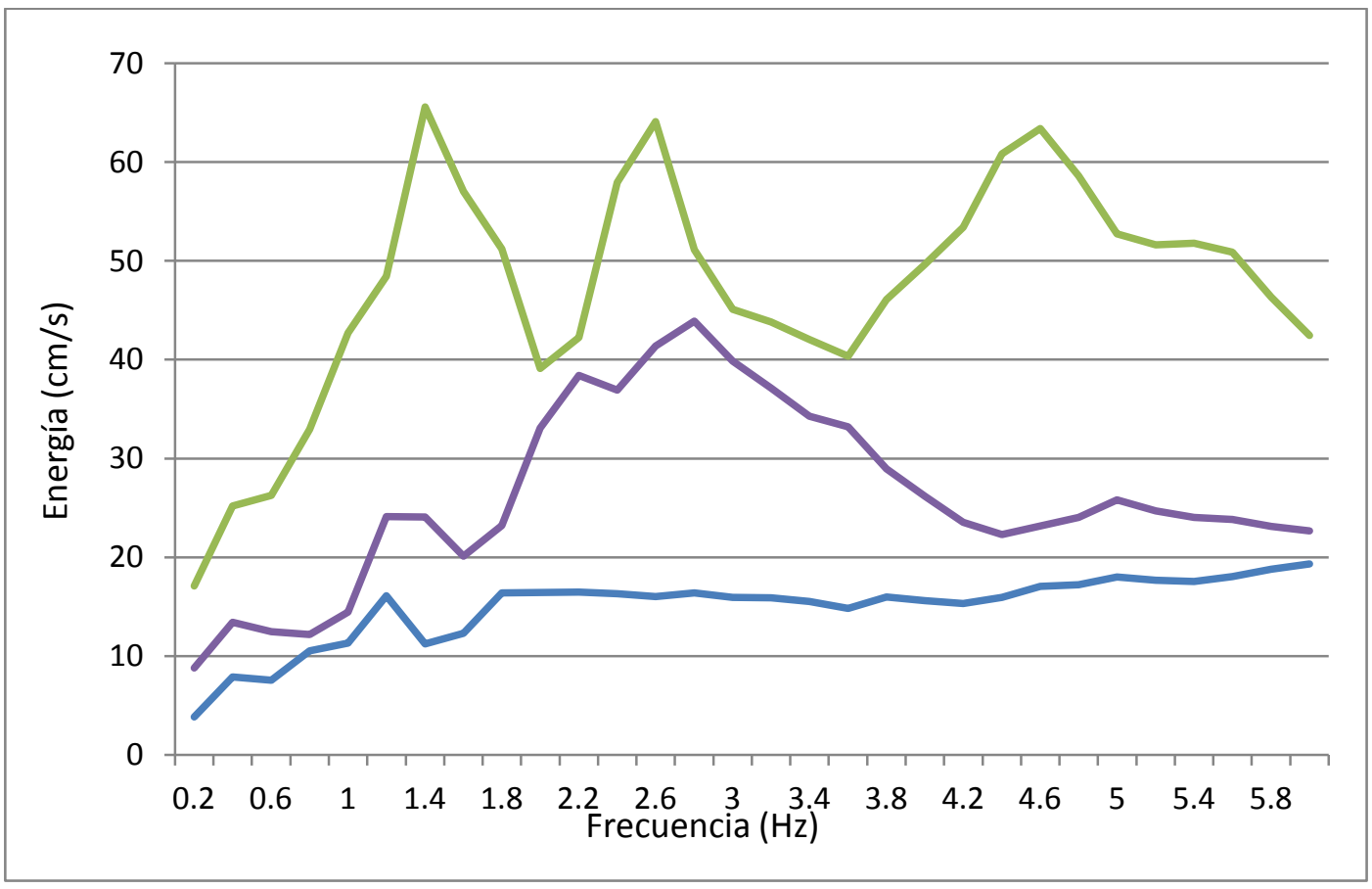

Figura 7.3b. Espectros de input de energía obtenidos tras aplicar la función de amplificación 


\section{Chana}

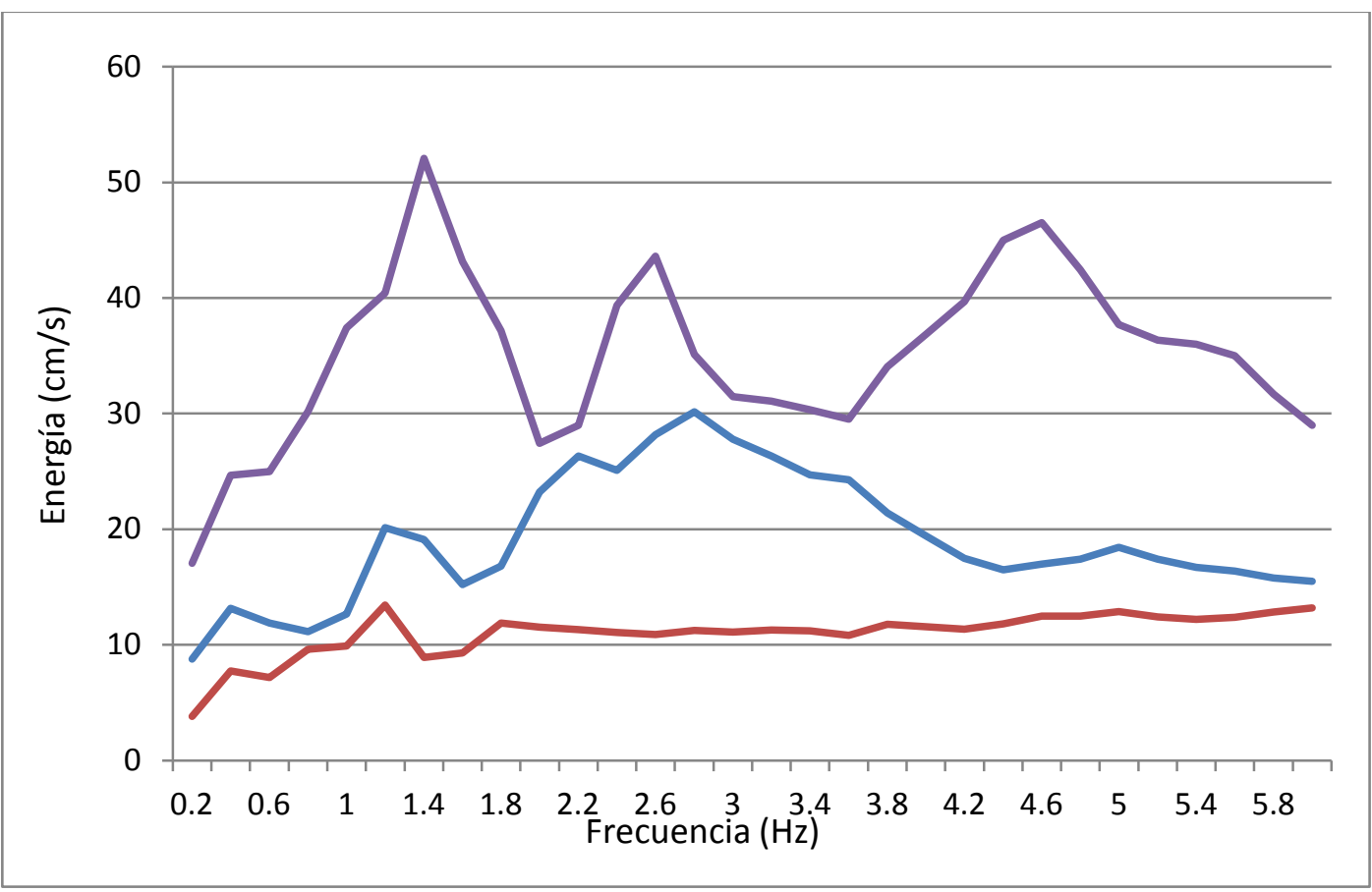

Figura 7.4a. Espectros de input de energía obtenidos antes de aplicar la función de amplificación

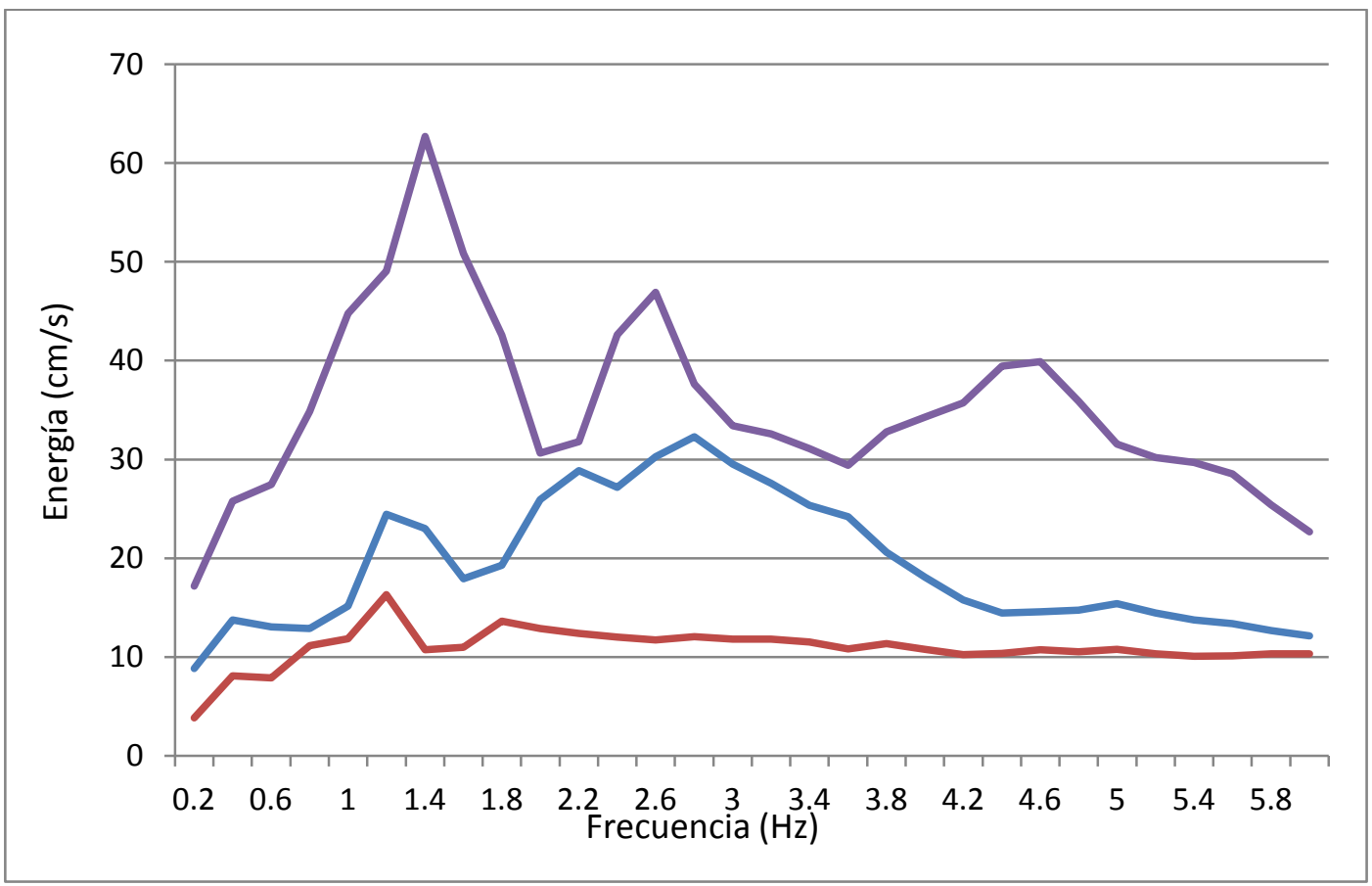

Figura 7.4b. Espectros de input de energía obtenidos tras aplicar la función de amplificación 


\section{Estadio de la Juventud}

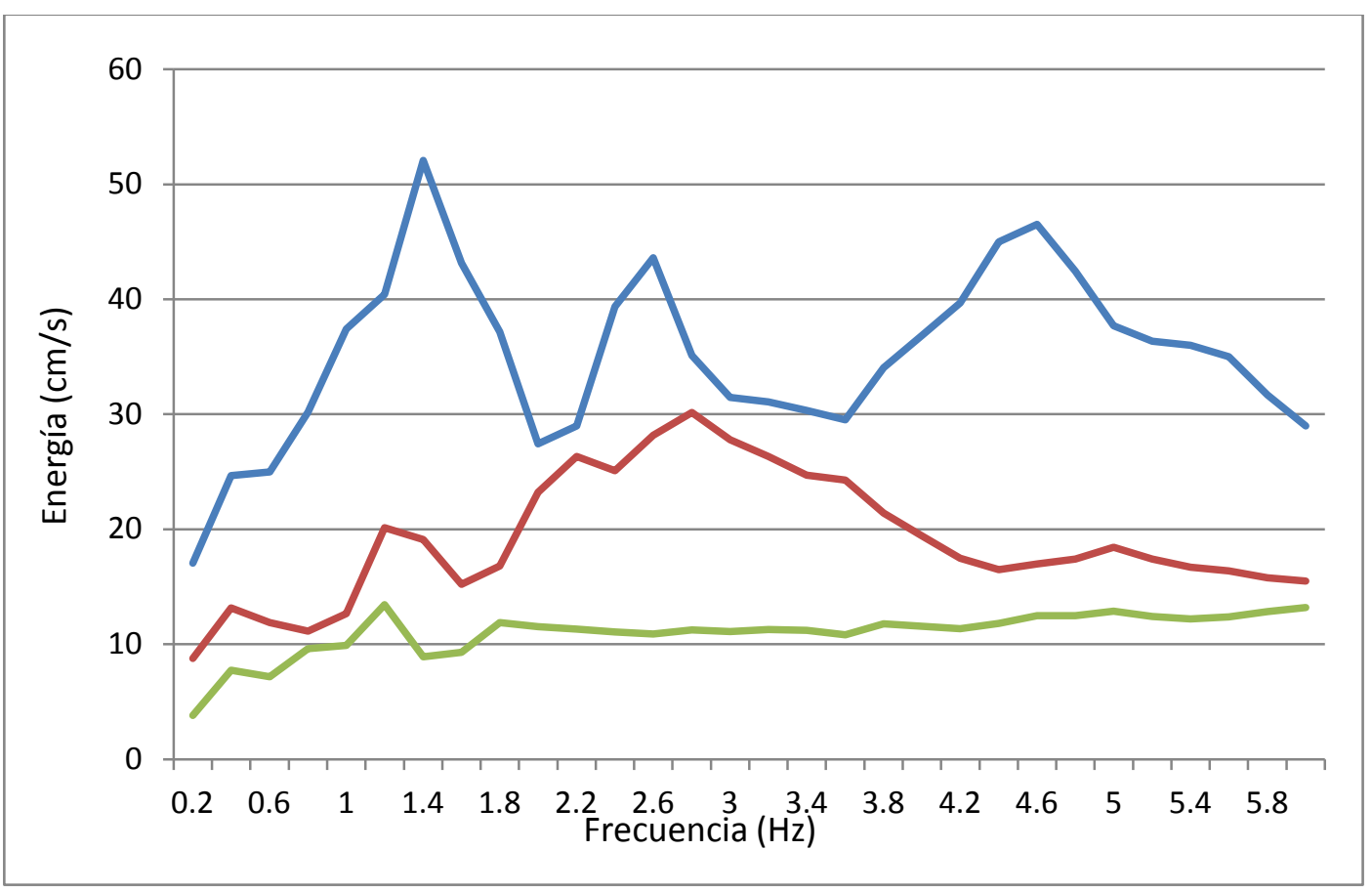

Figura 7.5a. Espectros de input de energía obtenidos antes de aplicar la función de amplificación

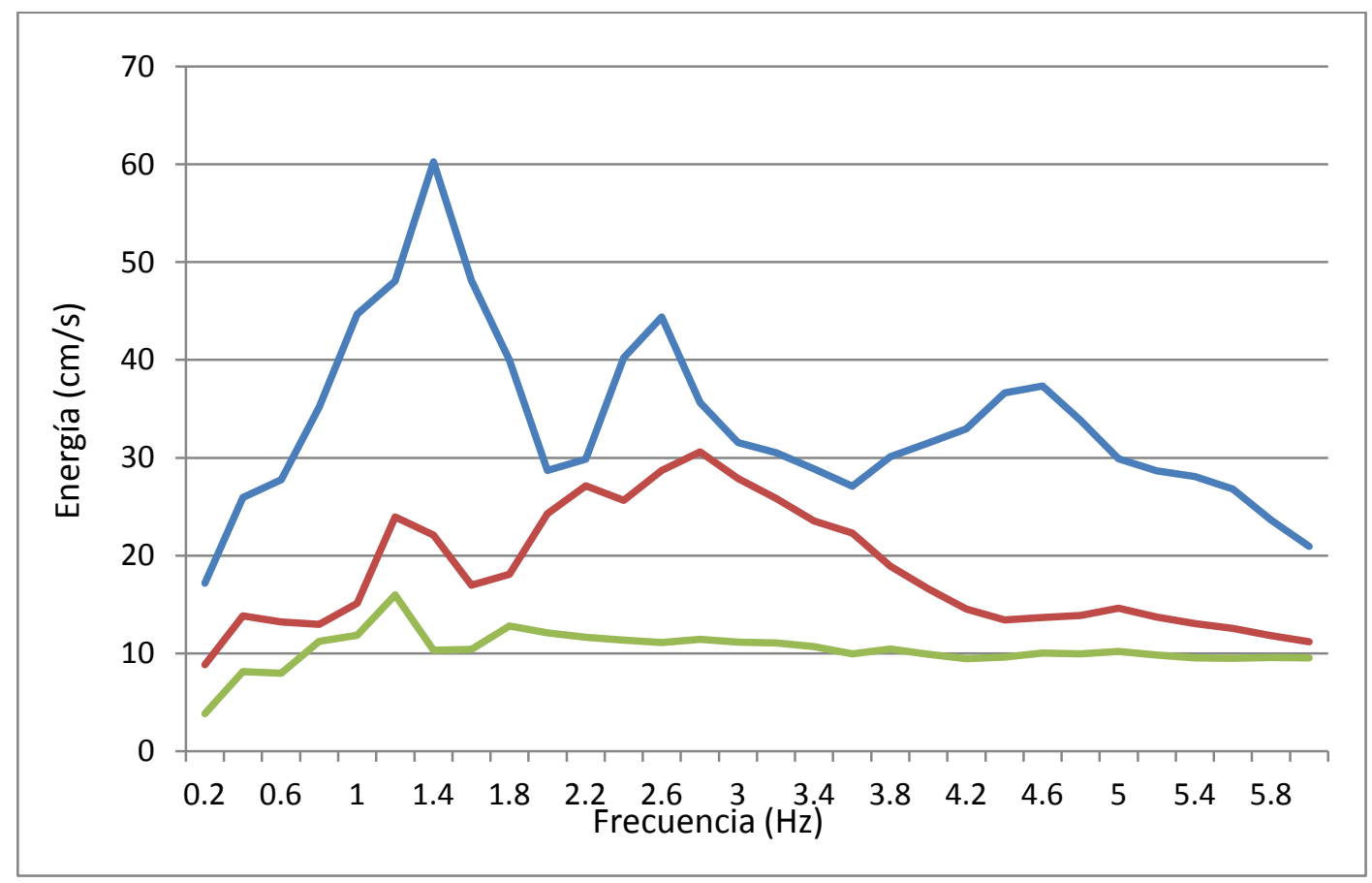

Figura 7.5b. Espectros de input de energía obtenidos tras aplicar la función de amplificación 


\section{Ferial}

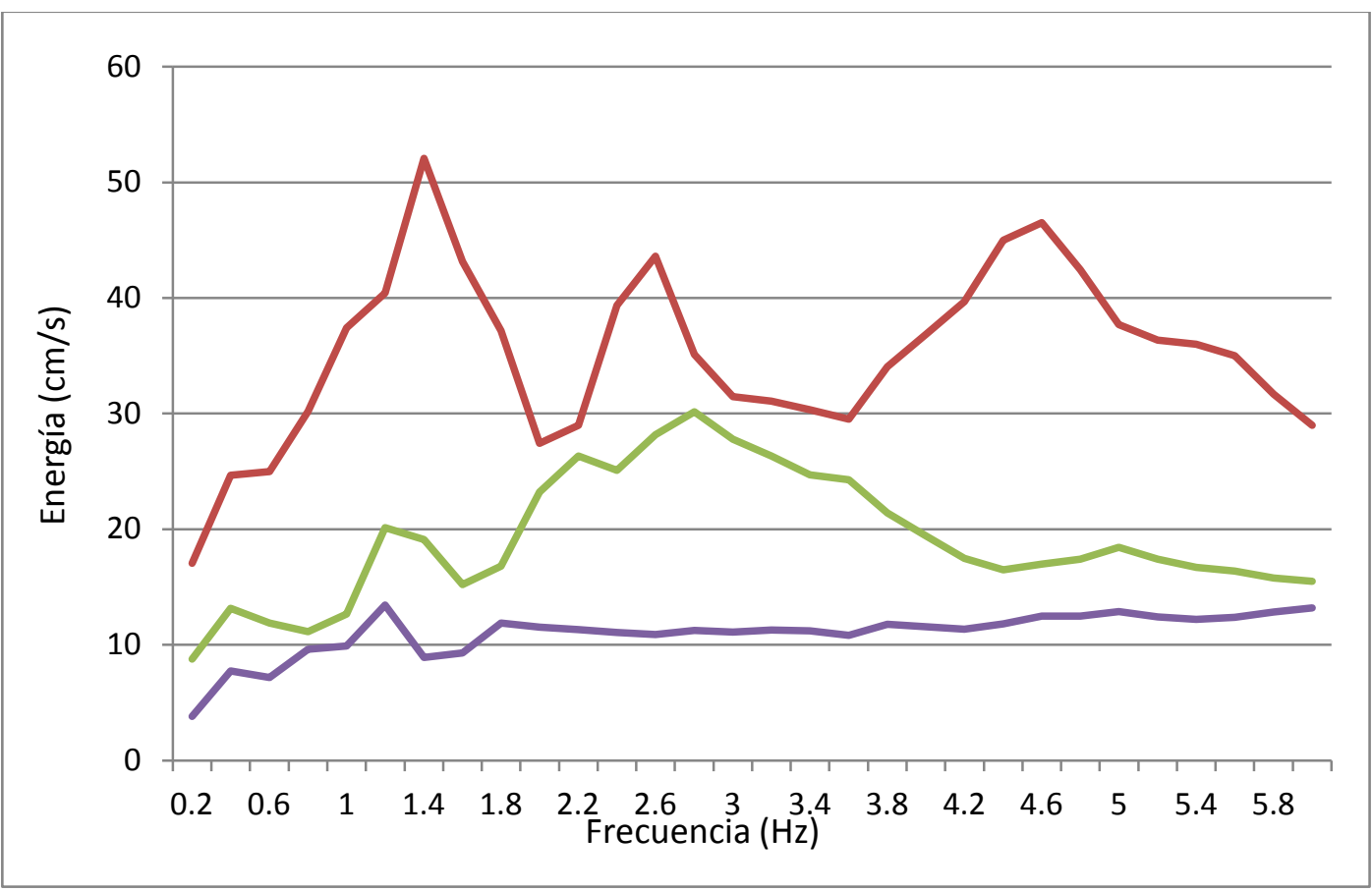

Figura 7.6a. Espectros de input de energía obtenidos antes de aplicar la función de amplificación

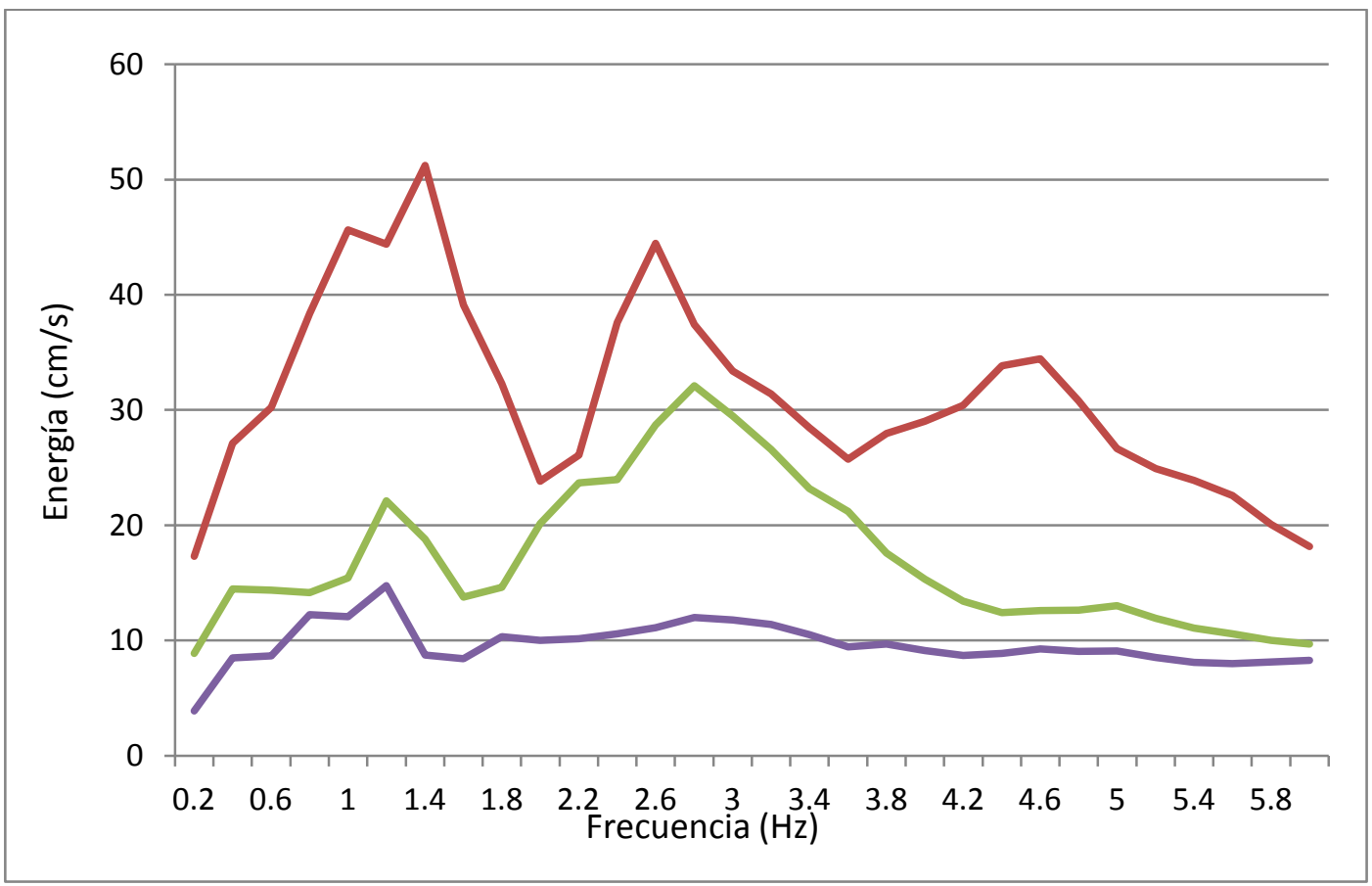

Figura 7.6b. Espectros de input de energía obtenidos tras aplicar la función de amplificación 


\section{Fuentenueva}

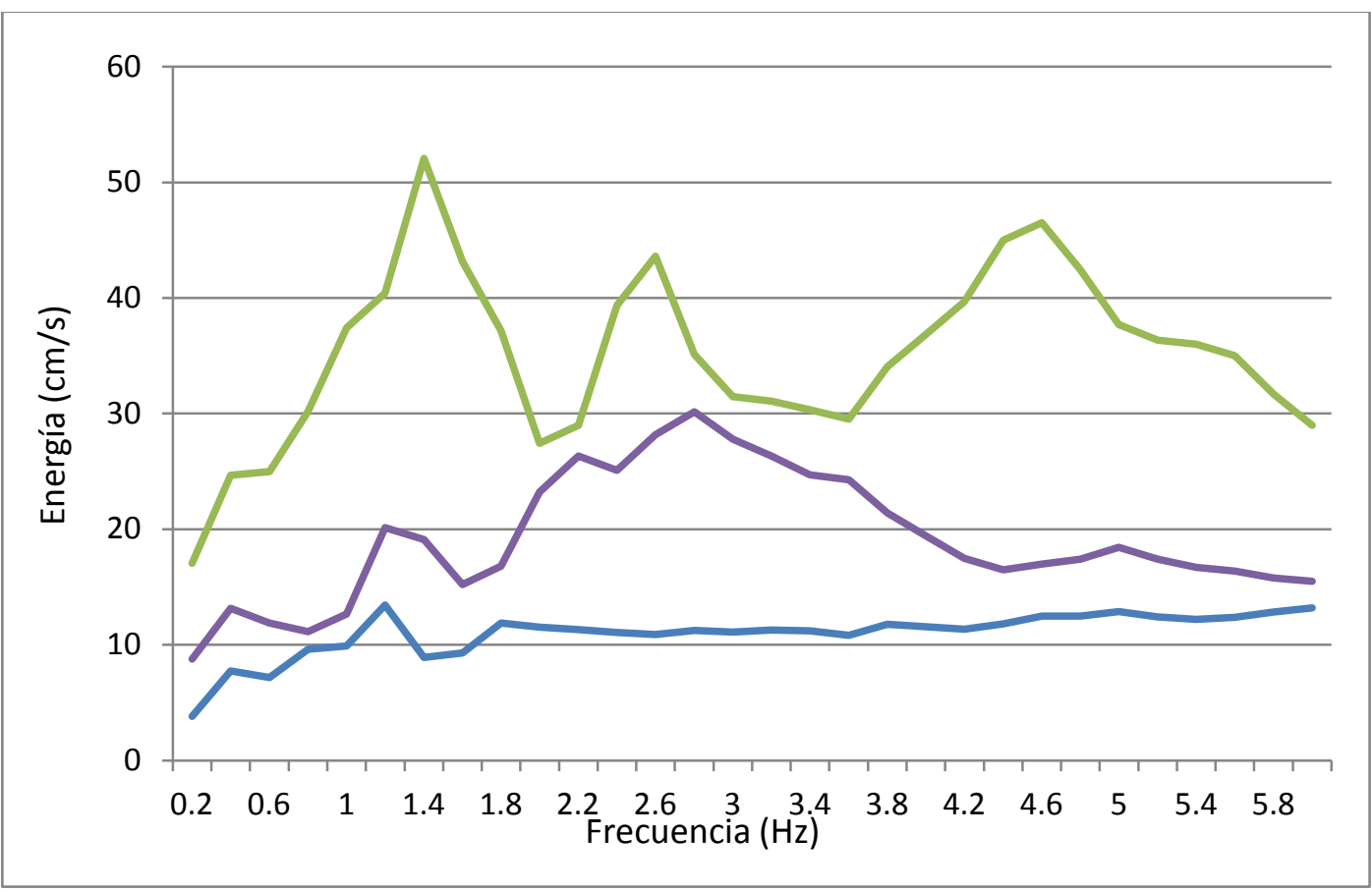

Figura 7.7a. Espectros de input de energía obtenidos antes de aplicar la función de amplificación

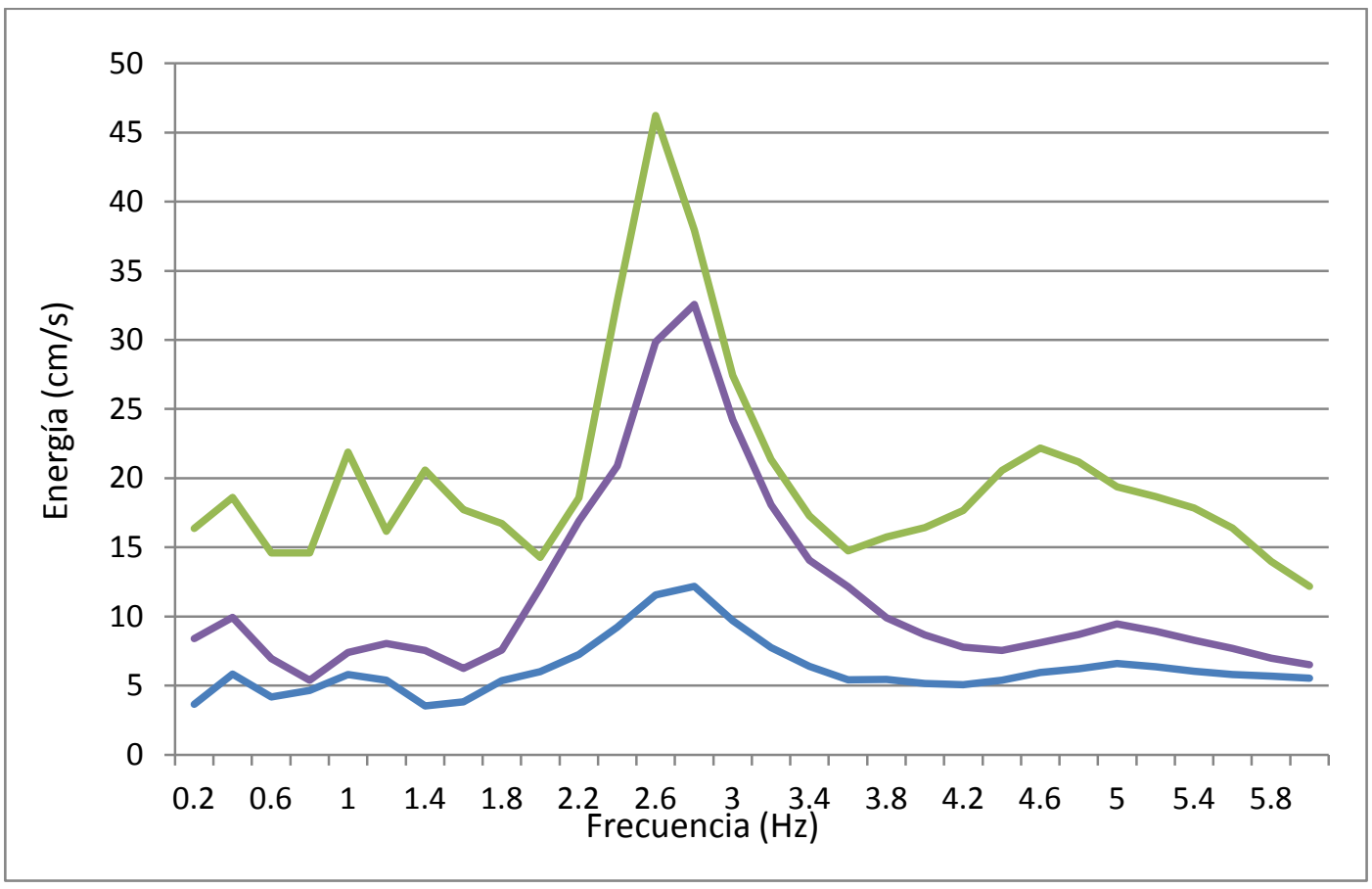

Figura 7.7b. Espectros de input de energía obtenidos tras aplicar la función de amplificación 


\section{Ciudad Deportiva Granada 74}

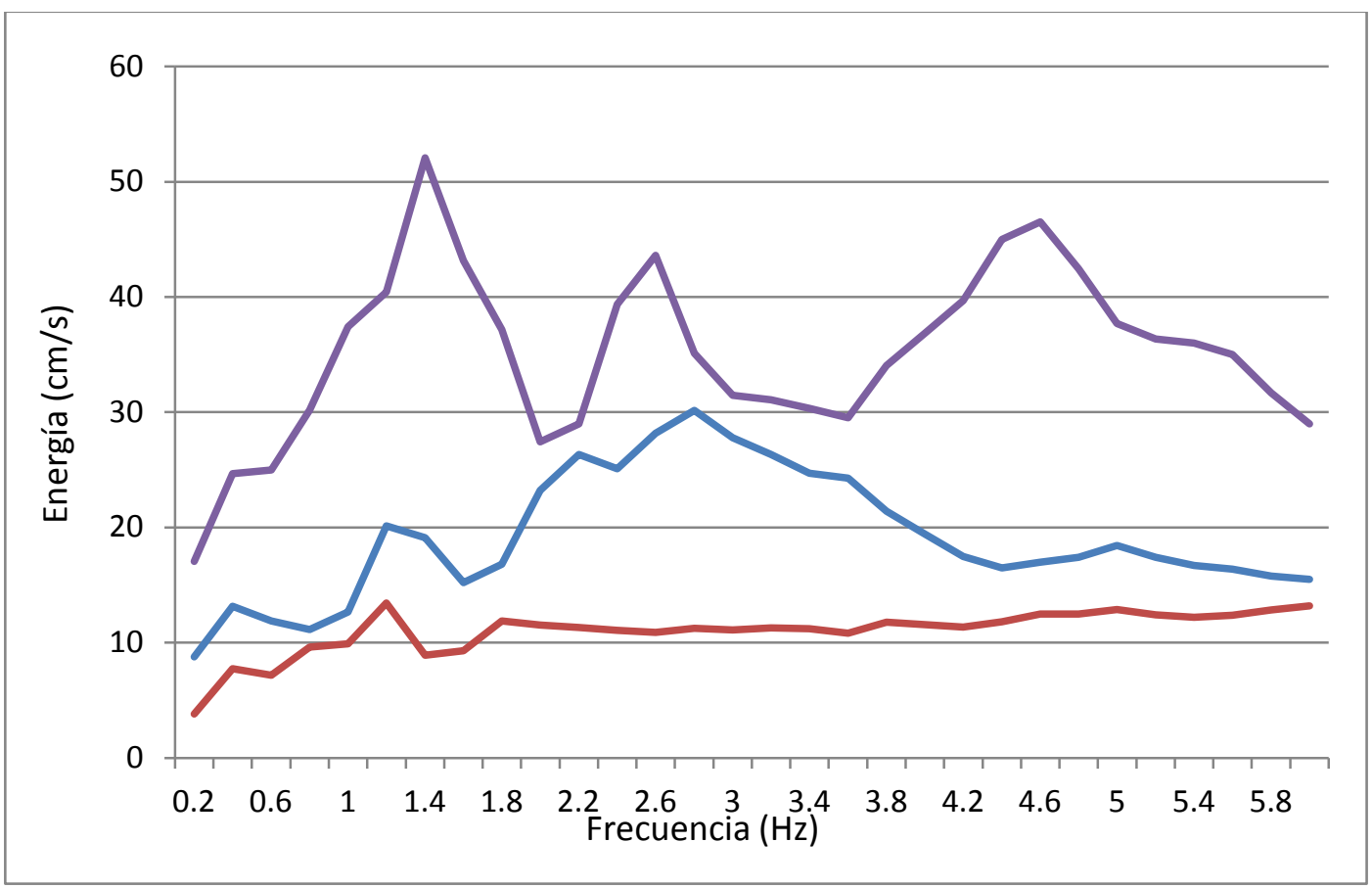

Figura 7.8a. Espectros de input de energía obtenidos antes de aplicar la función de amplificación

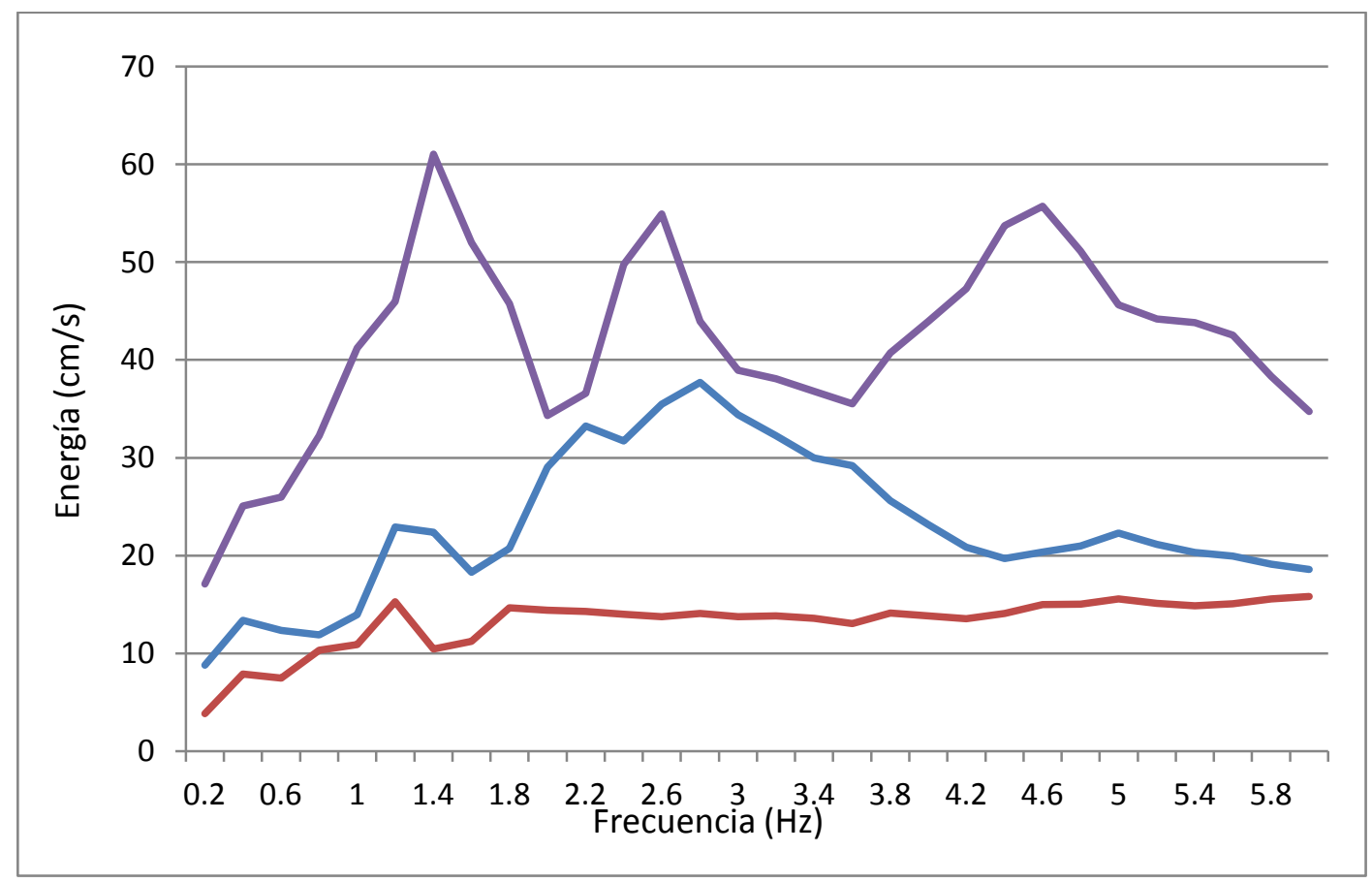

Figura 7.8b. Espectros de input de energía obtenidos tras aplicar la función de amplificació 


\section{Calle Náyades}

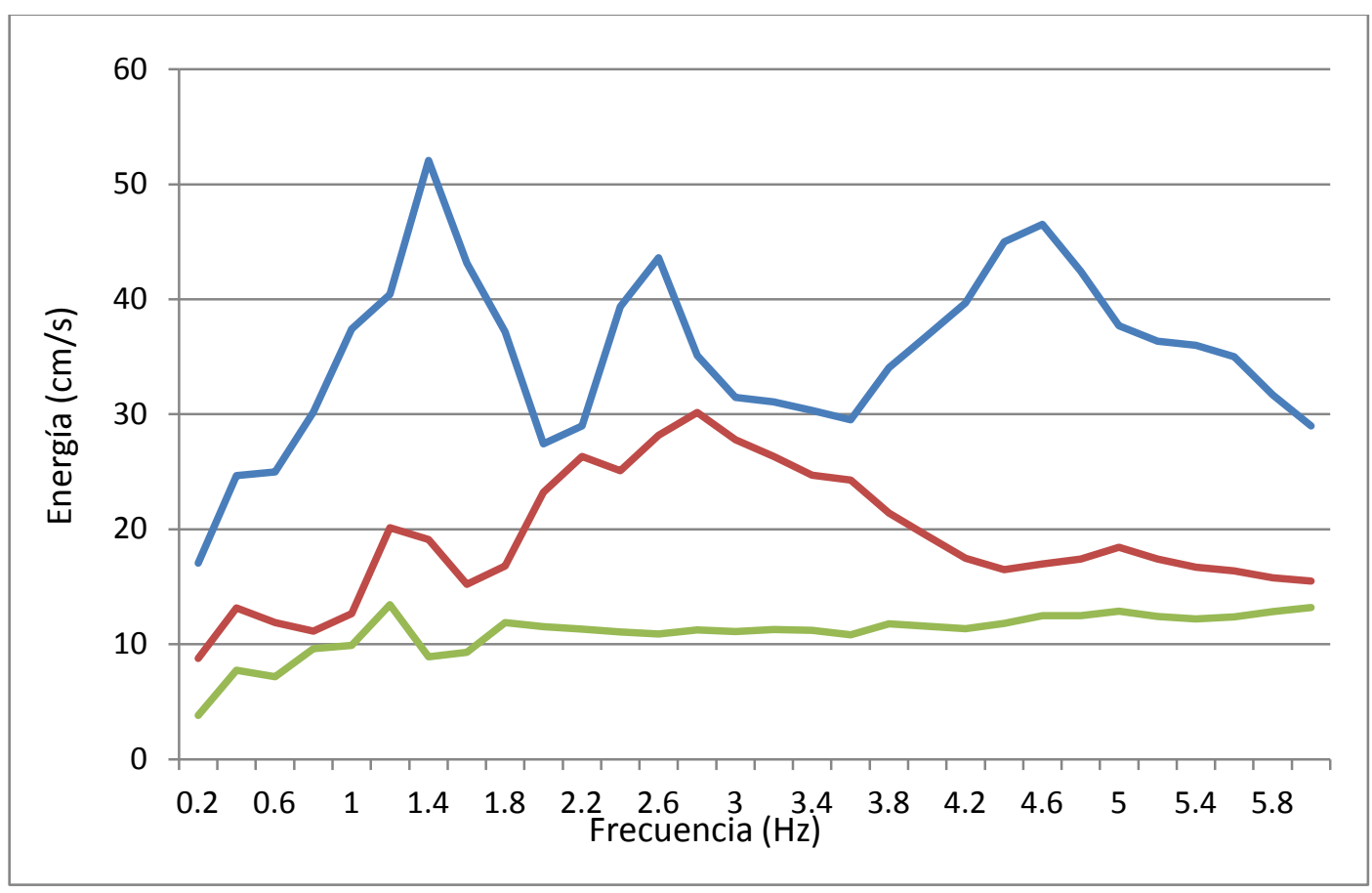

Figura 7.9a. Espectros de input de energía obtenidos antes de aplicar la función de amplificación

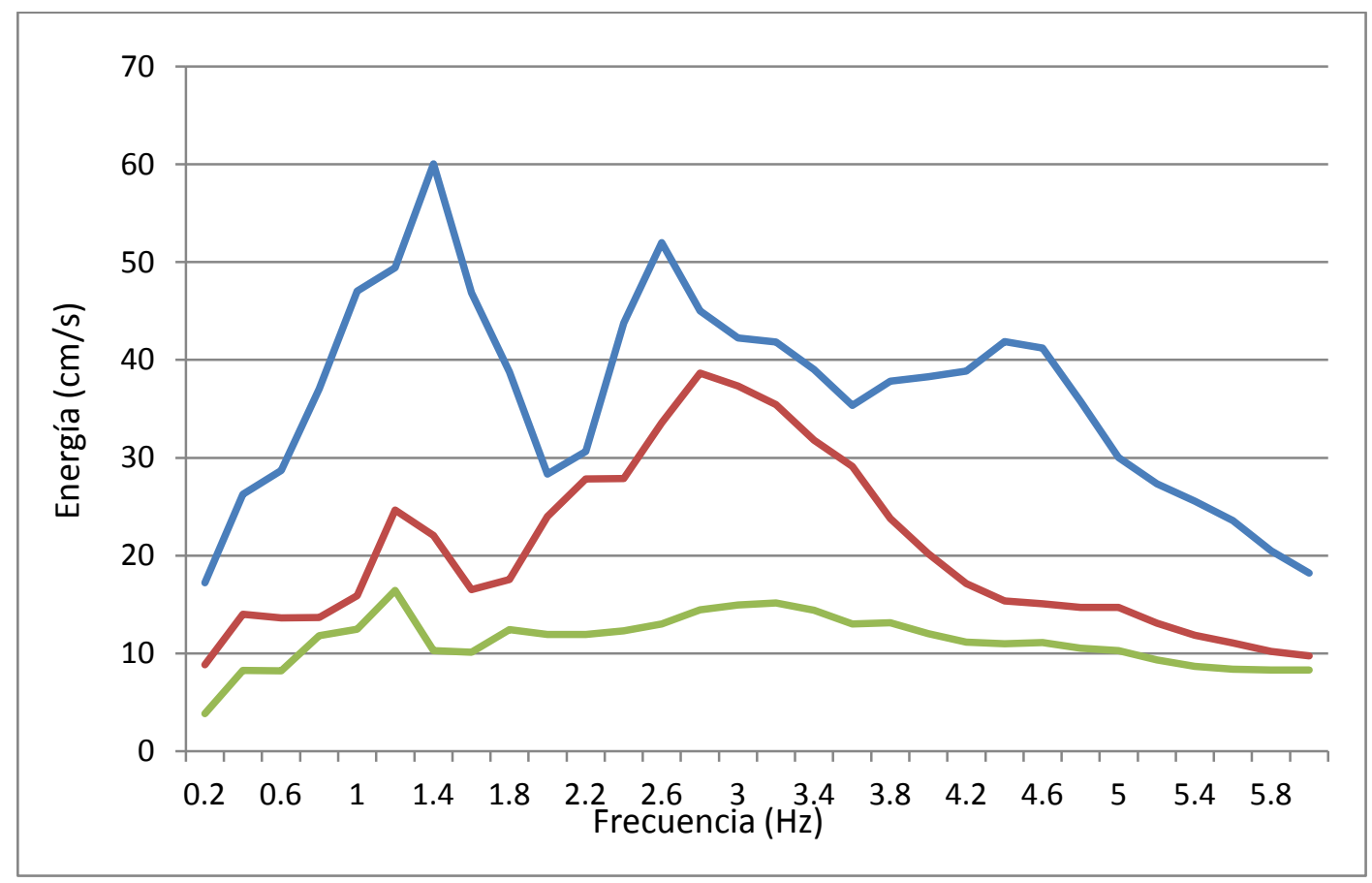

Figura 7.9b. Espectros de input de energía obtenidos tras aplicar la función de amplificación 


\section{Parque de las ciencias}

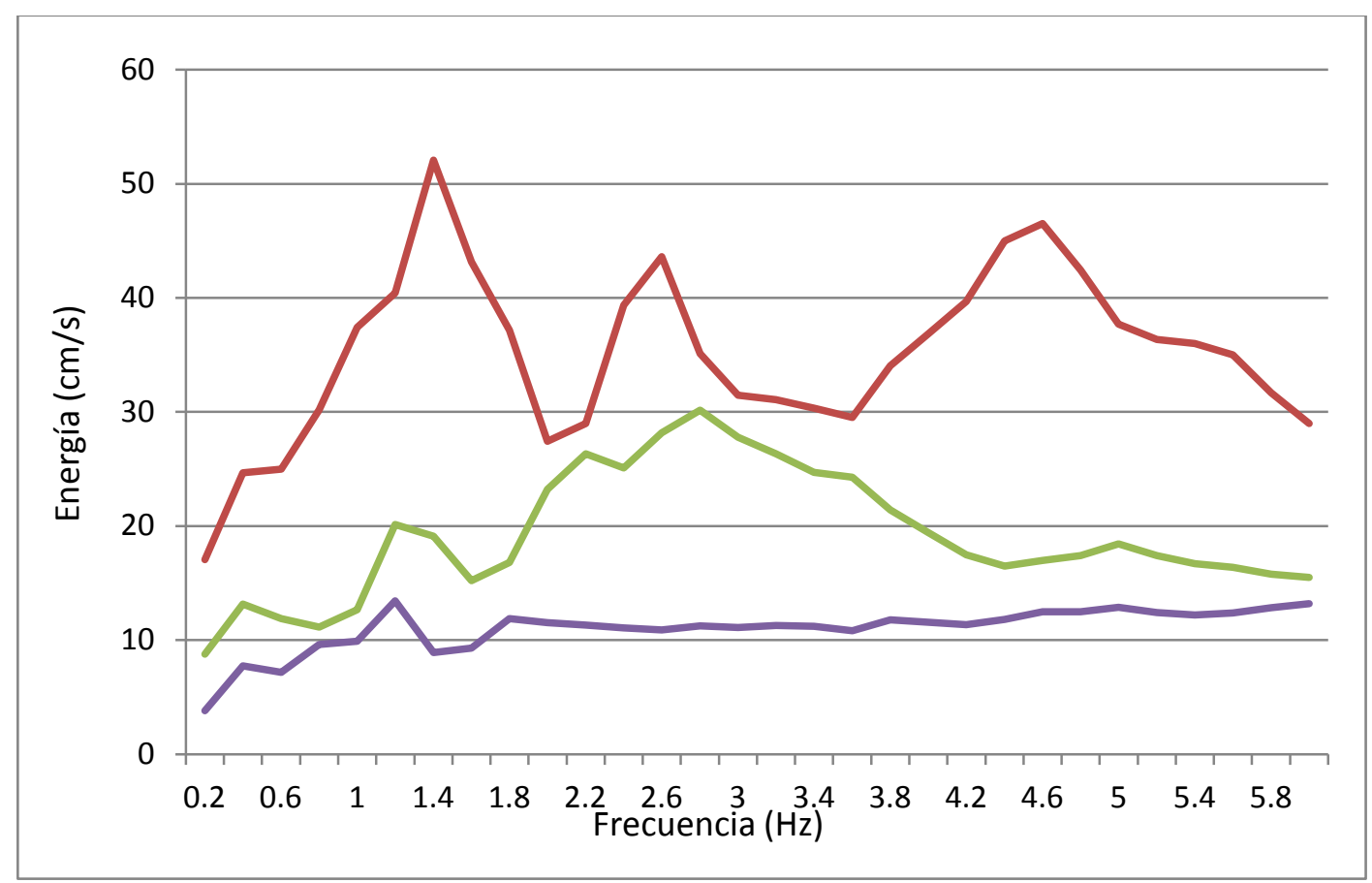

Figura 7.10a. Espectros de input de energía obtenidos antes de aplicar la función de amplificación

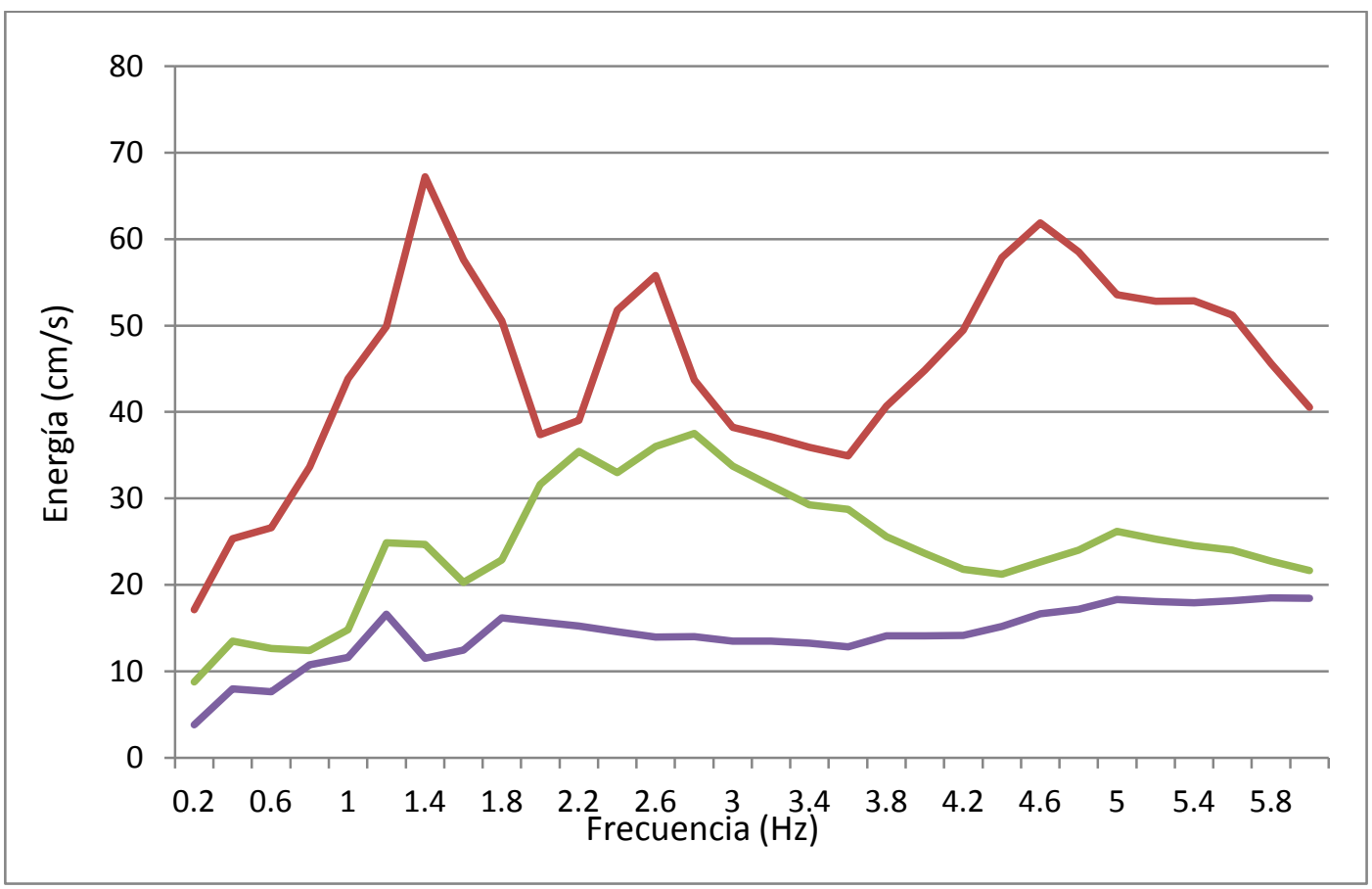

Figura 7.10b. Espectros de input de energía obtenidos tras aplicar la función de amplificación 


\section{Antigua fábrica de Sanders}

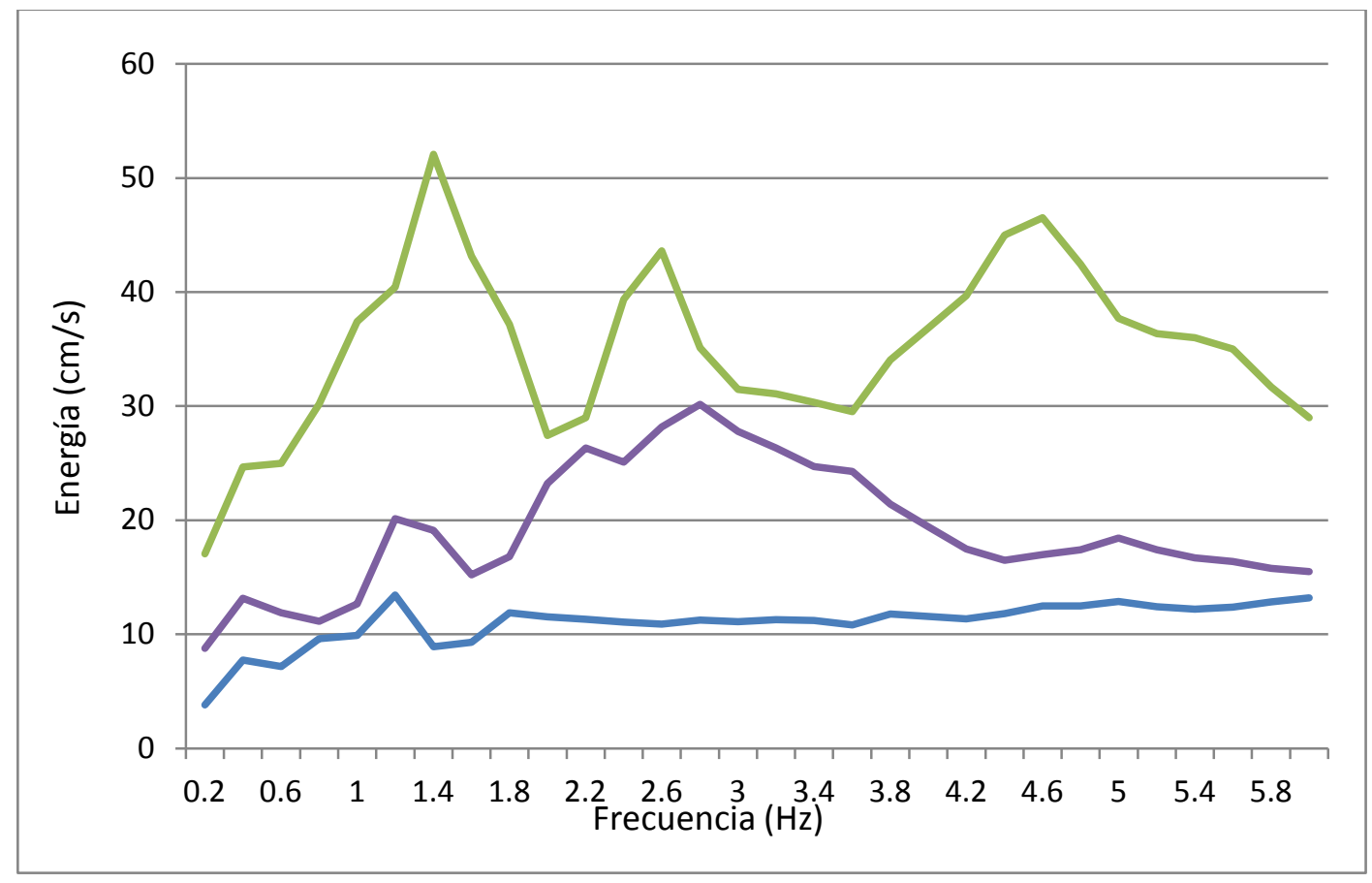

Figura 7.11a Espectros de input de energía obtenidos antes de aplicar la función de amplificación

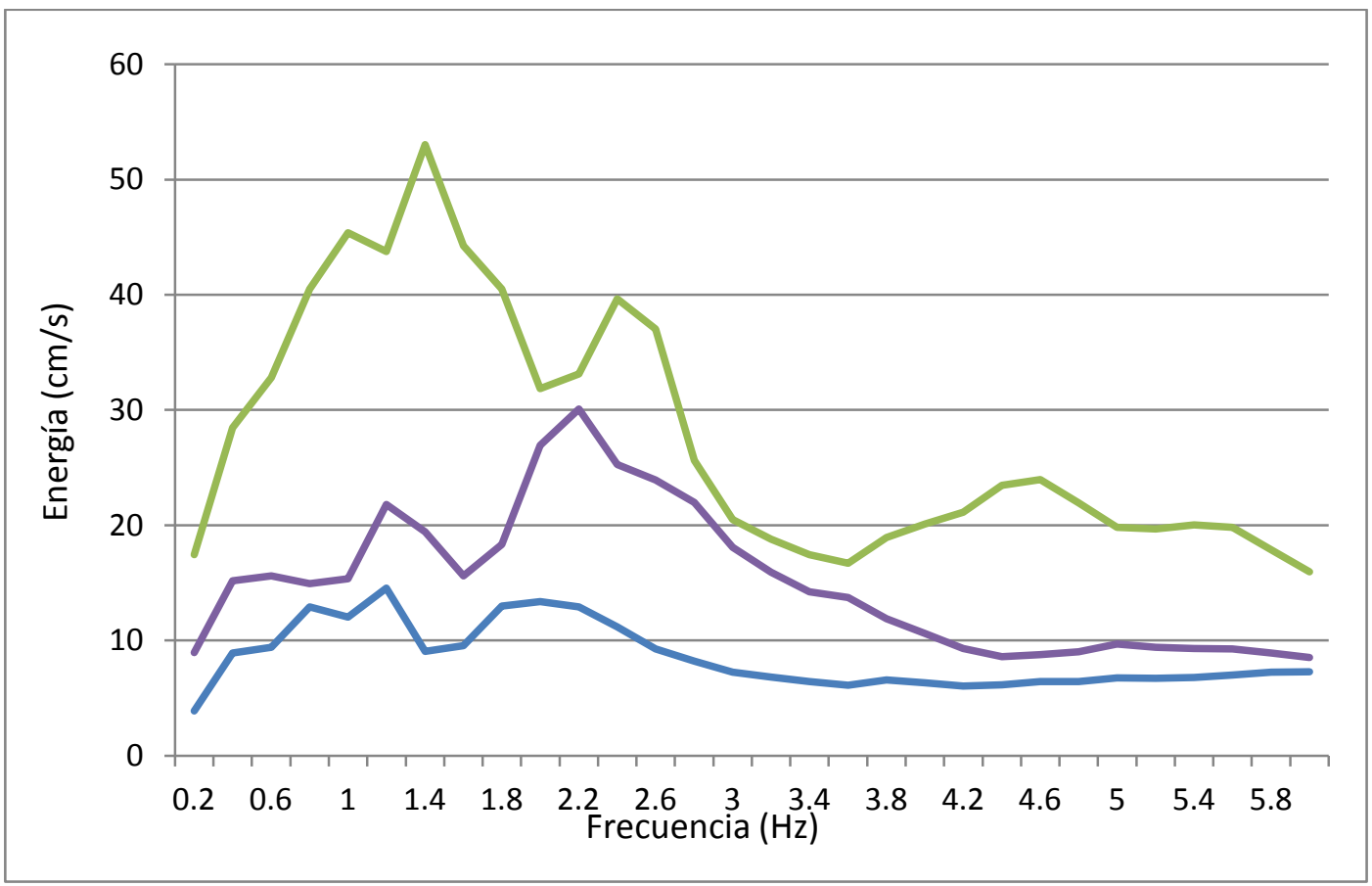

Figura 7.11b. Espectros de input de energía obtenidos tras aplicar la función de amplificación 


\section{Vega de Granada}

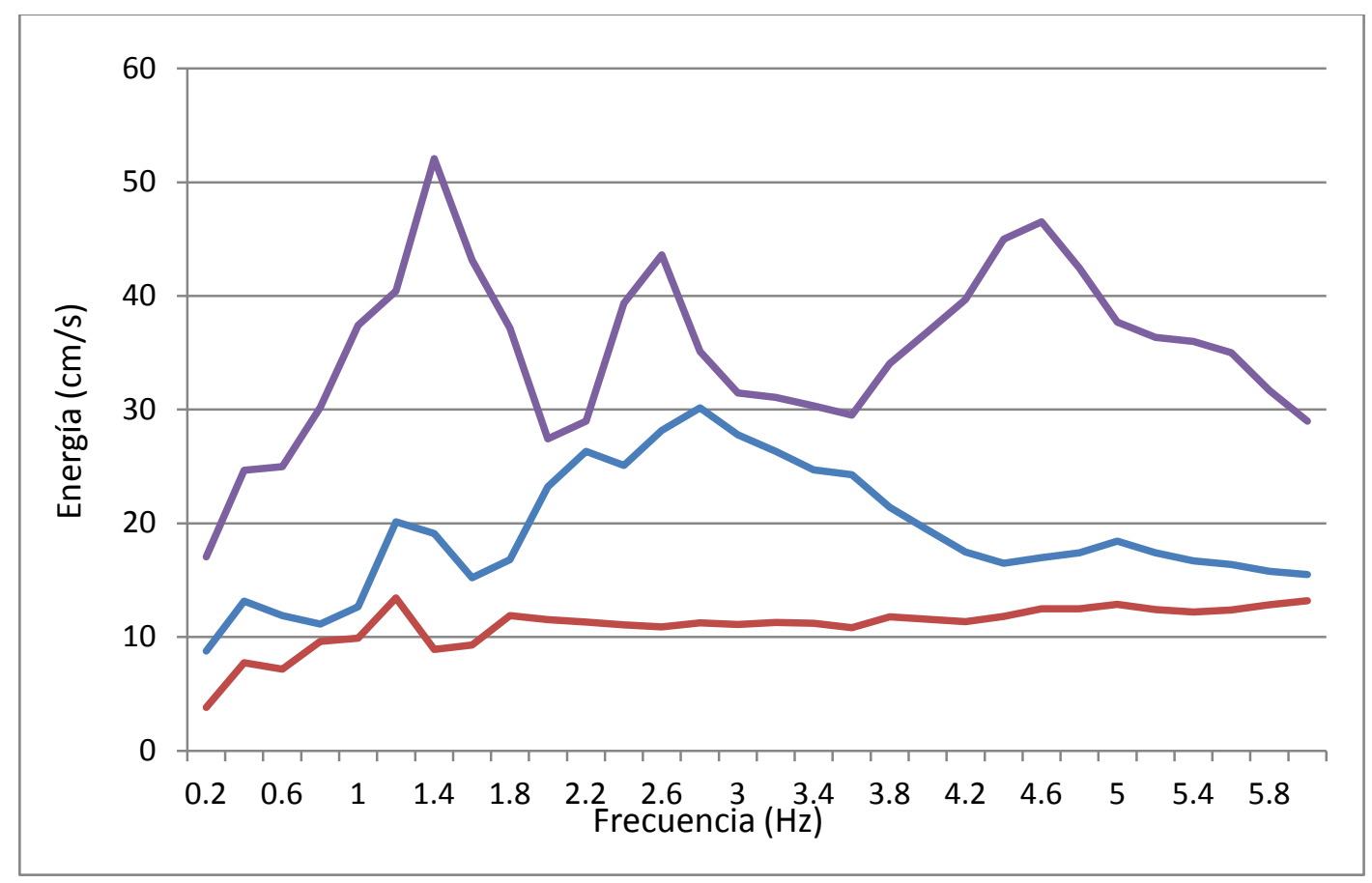

Figura 7.12a. Espectros de input de energía obtenidos antes de aplicar la función de amplificación

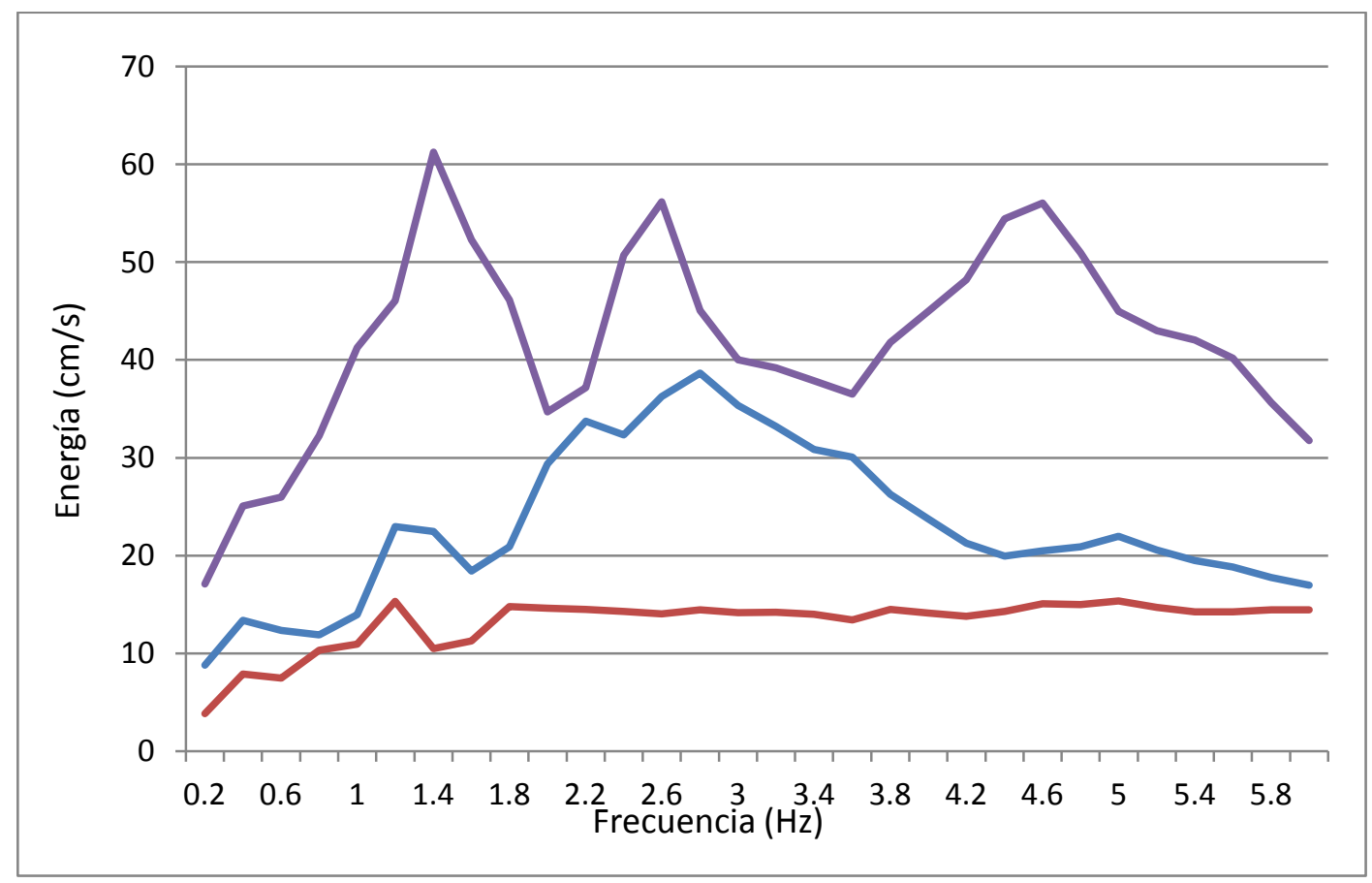

Figura 7.12b. Espectros de input de energía obtenidos tras aplicar la función de amplificación 


\section{Zaidín}

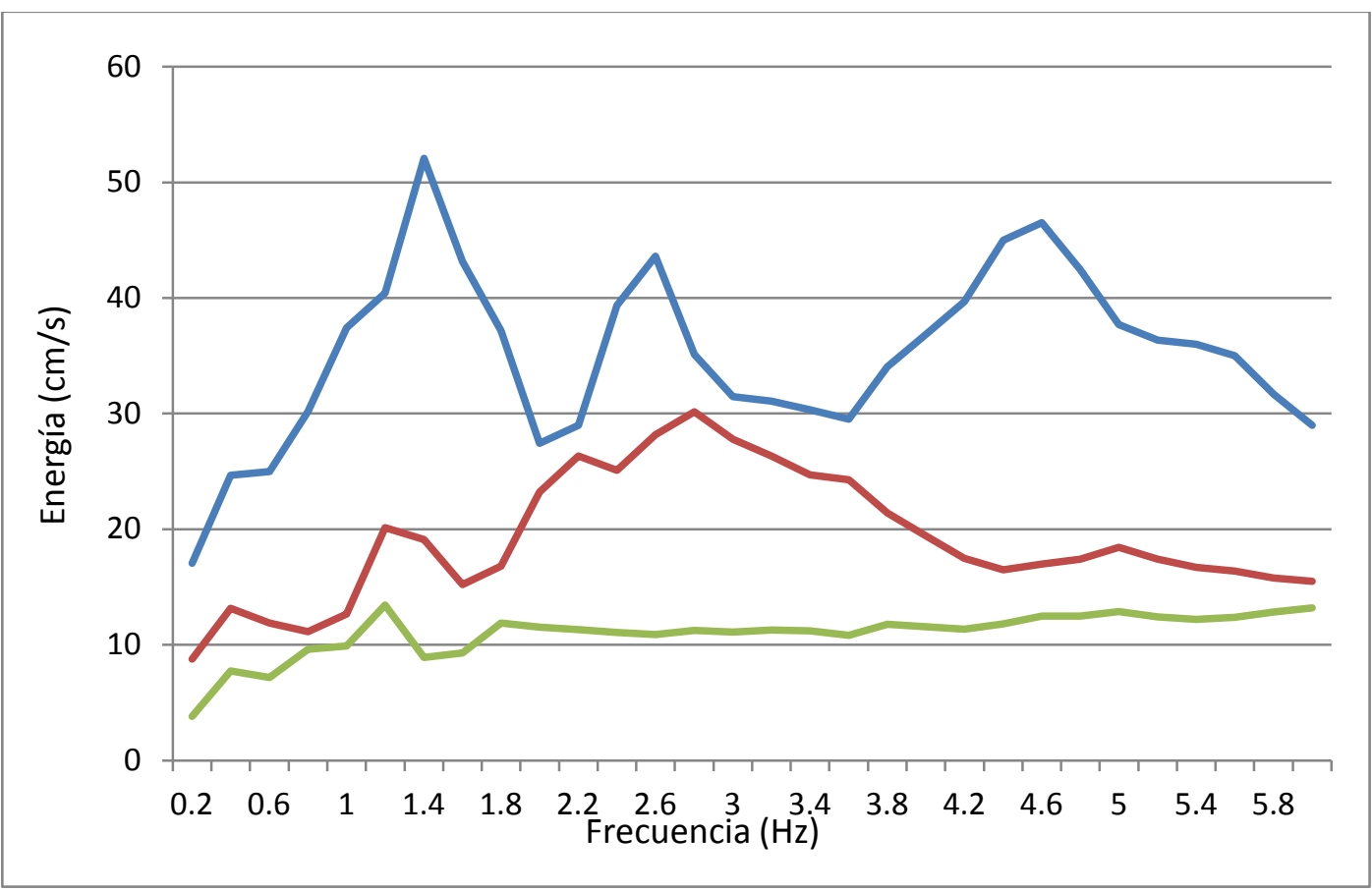

Figura 7.13a Espectros de input de energía obtenidos antes de aplicar la función de amplificación

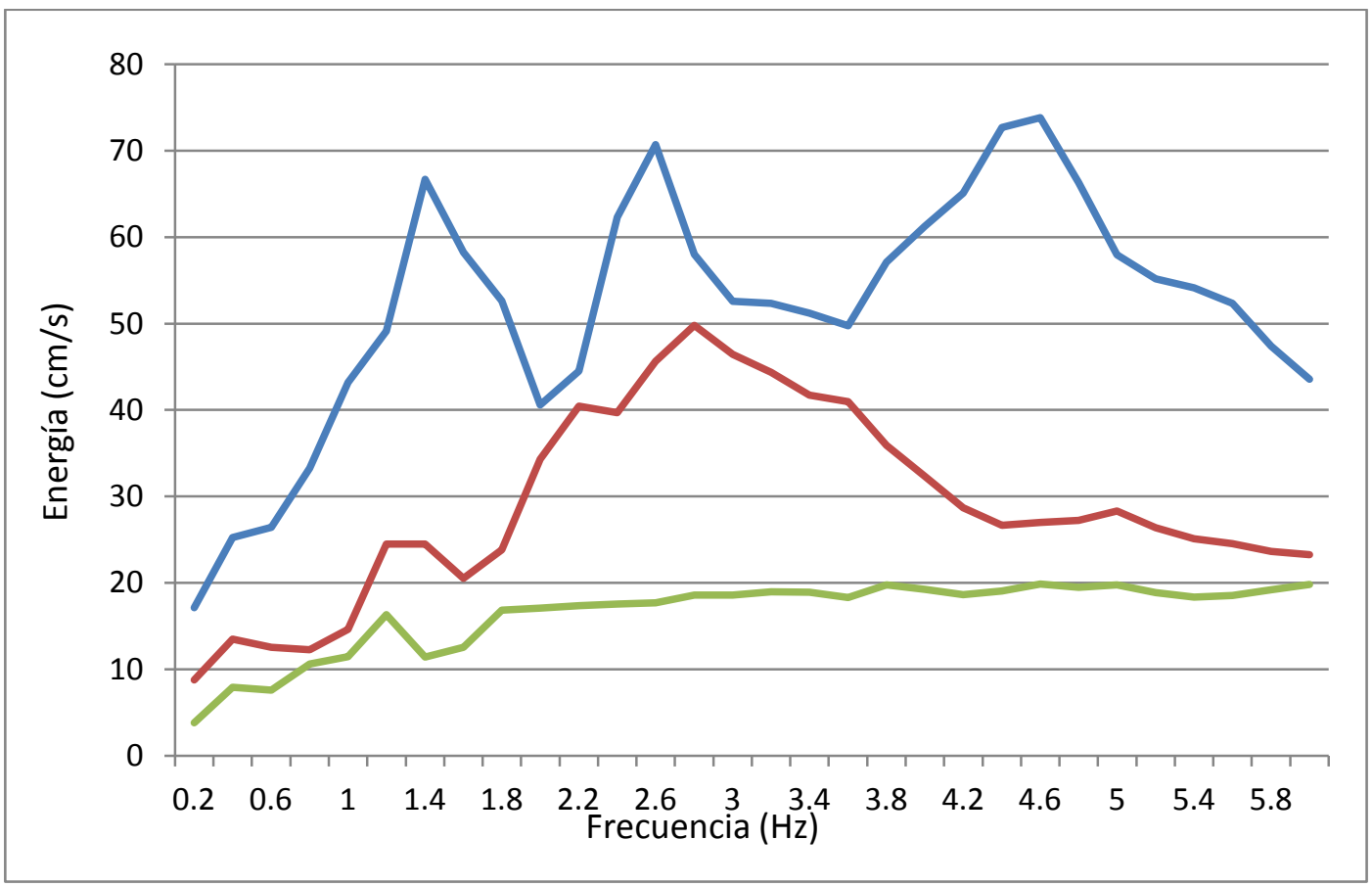

Figura 7.13b. Espectros de input de energía obtenidos tras aplicar la función de amplificación 


\subsection{Terremoto de "Basso Tirreno" (Italia)}

Al igual que en el caso anterior, las características del registro de este sismo se recogen en el Apéndice 2. En este caso ser han usado solo dos estaciones de registro. Los resultados obtenidos en cada una de las estaciones se presentan en las figuras 7.14 a 7.26.

\section{Aynadamar}

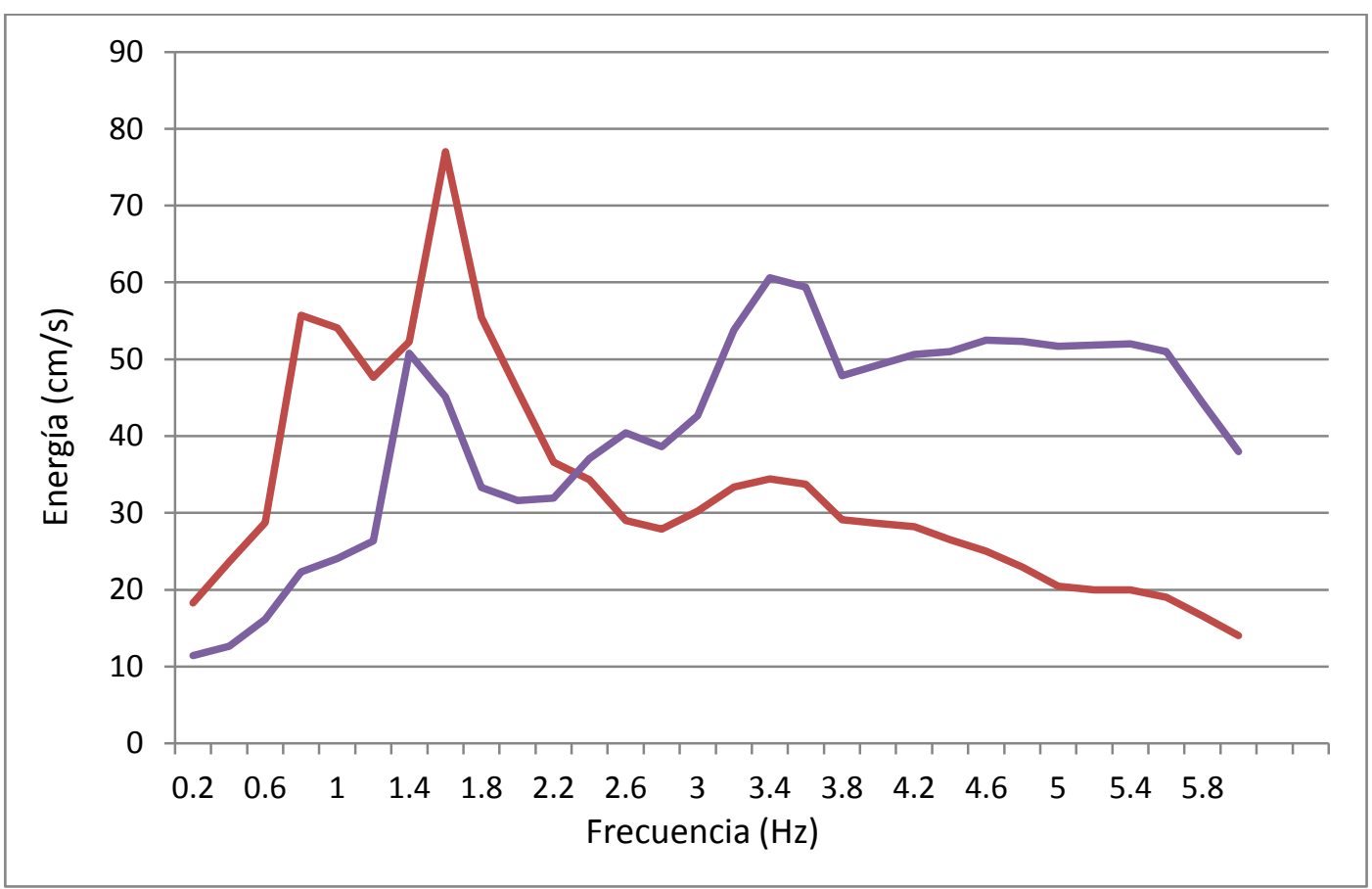

Figura 7.14a.Espectros de input de energía obtenidos antes de aplicar la función de amplificación

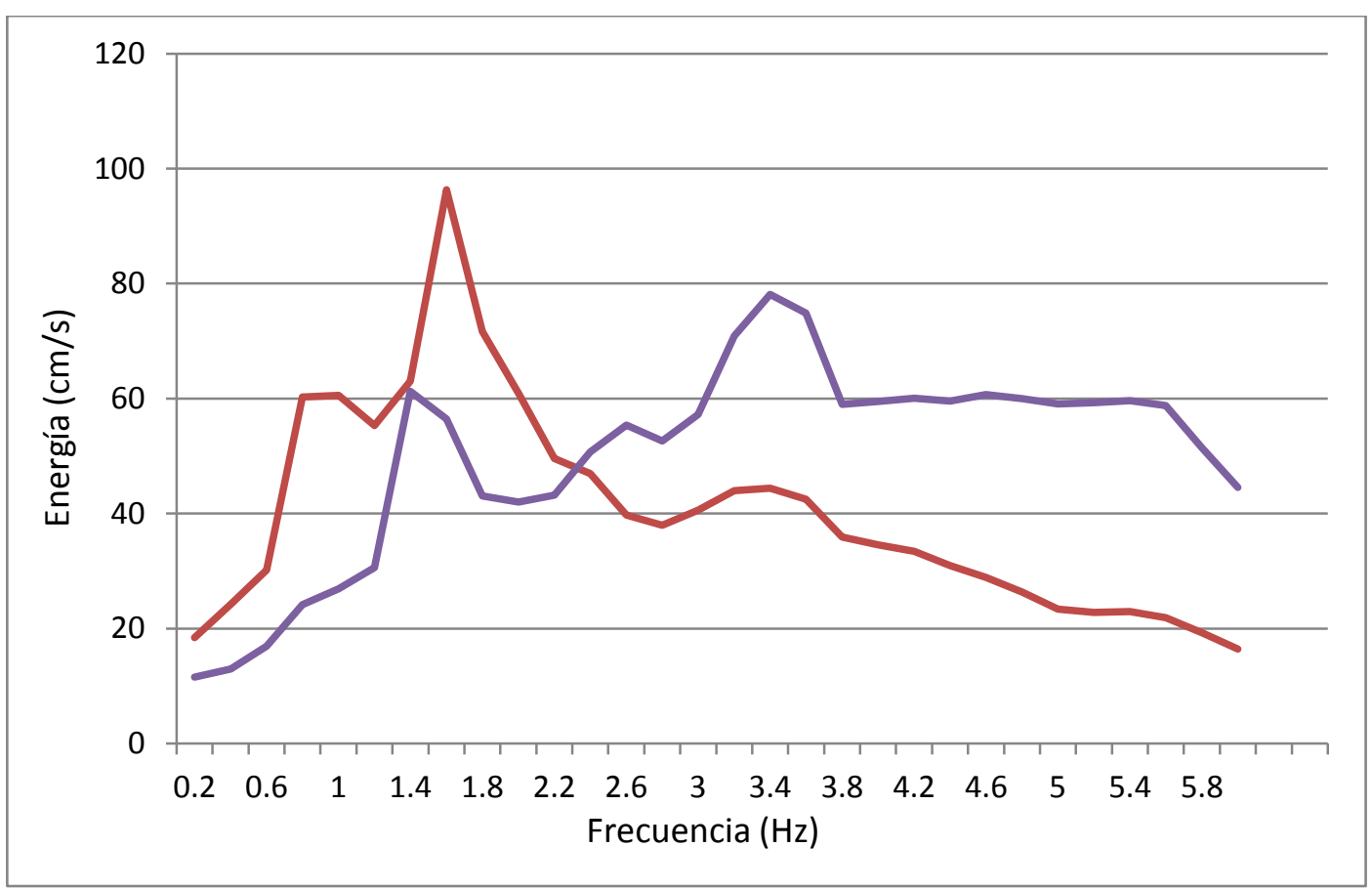

Figura 7.14b. Espectros de input de energía obtenidos tras aplicar la función de amplificación 


\section{Beiro}

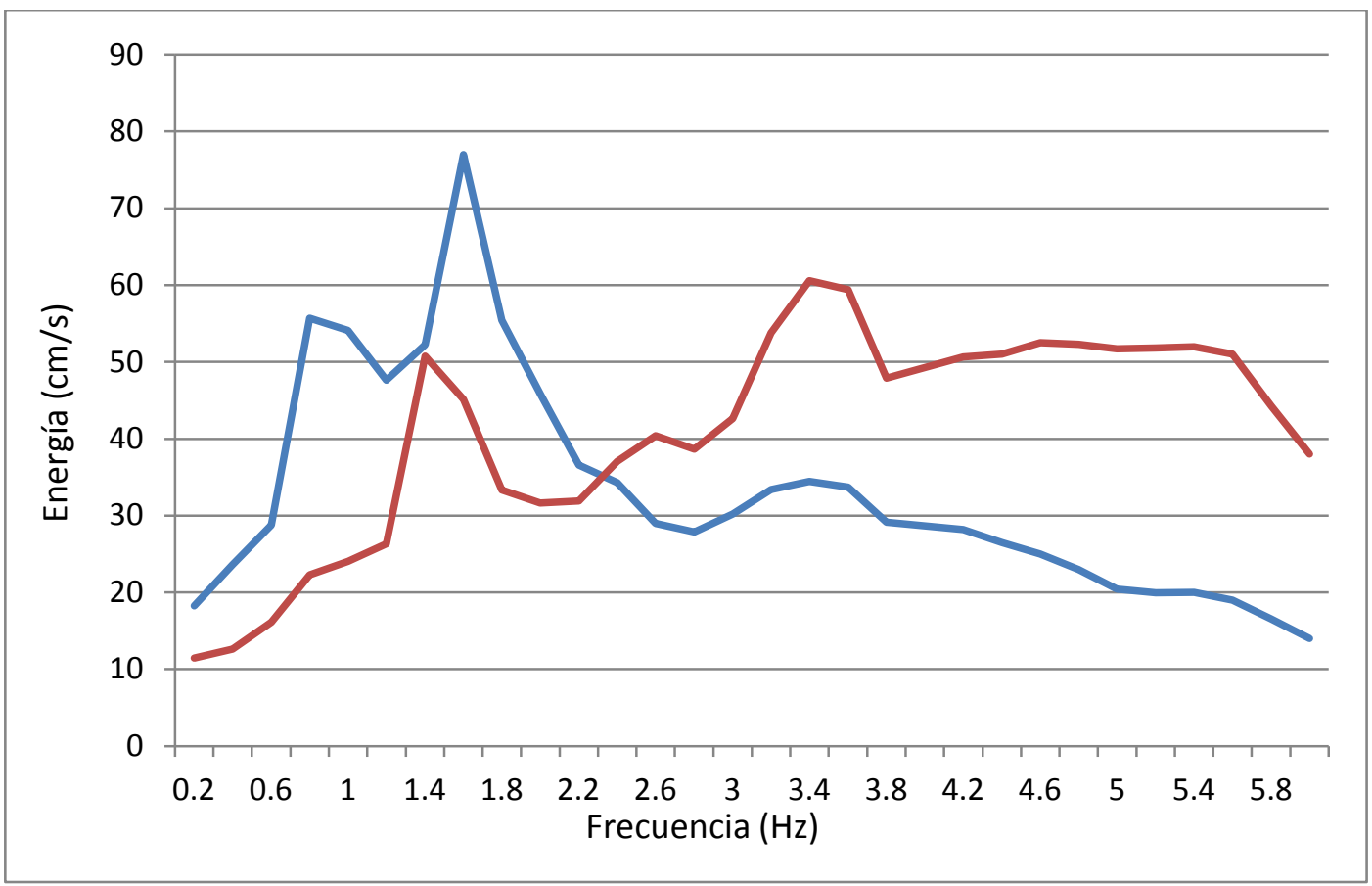

Figura 7.15a. Espectros de input de energía obtenidos antes de aplicar la función de amplificación

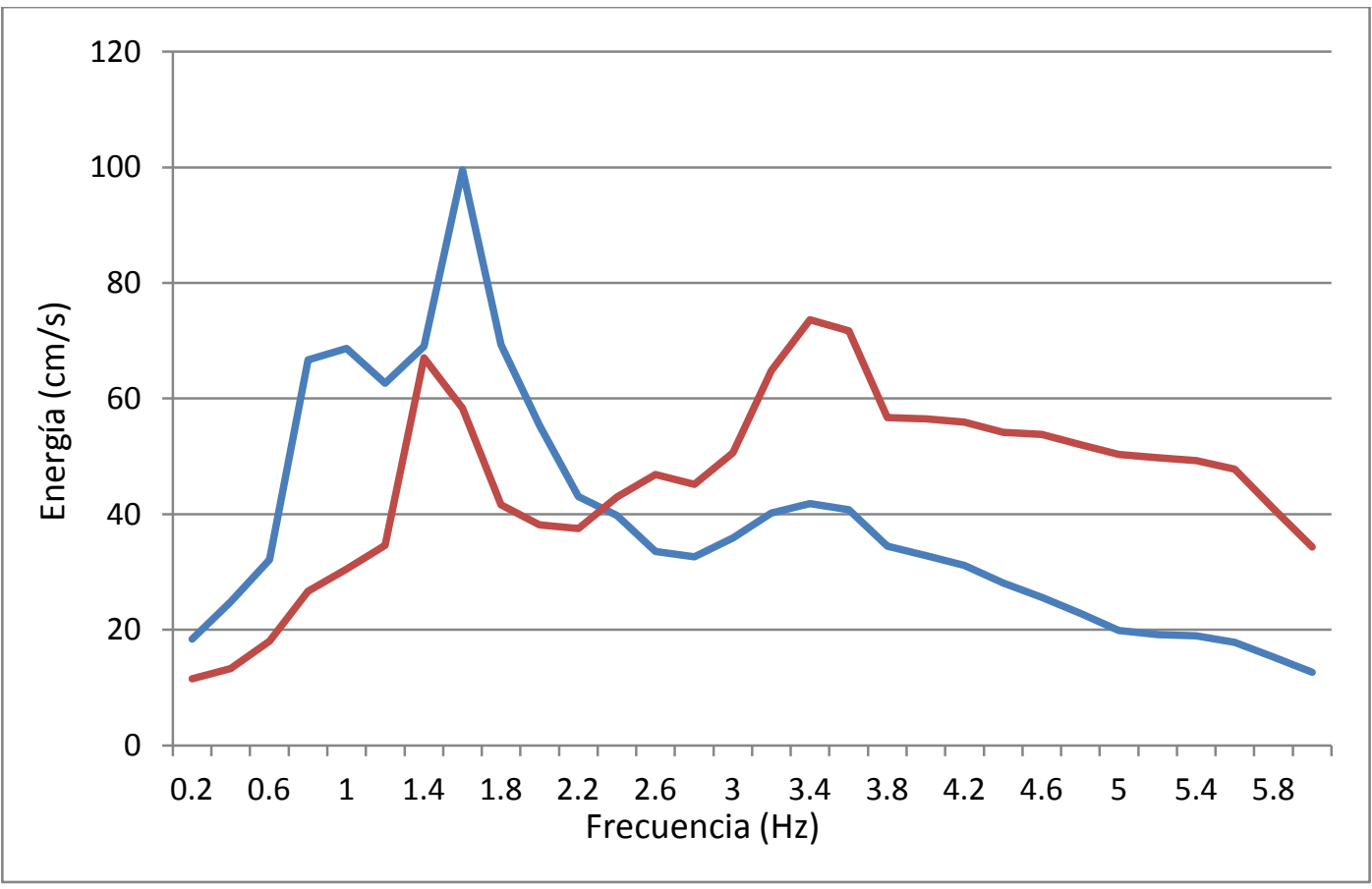

Figura 7.15b. Espectros de input de energía obtenidos tras aplicar la función de amplificación 
Bola de Oro

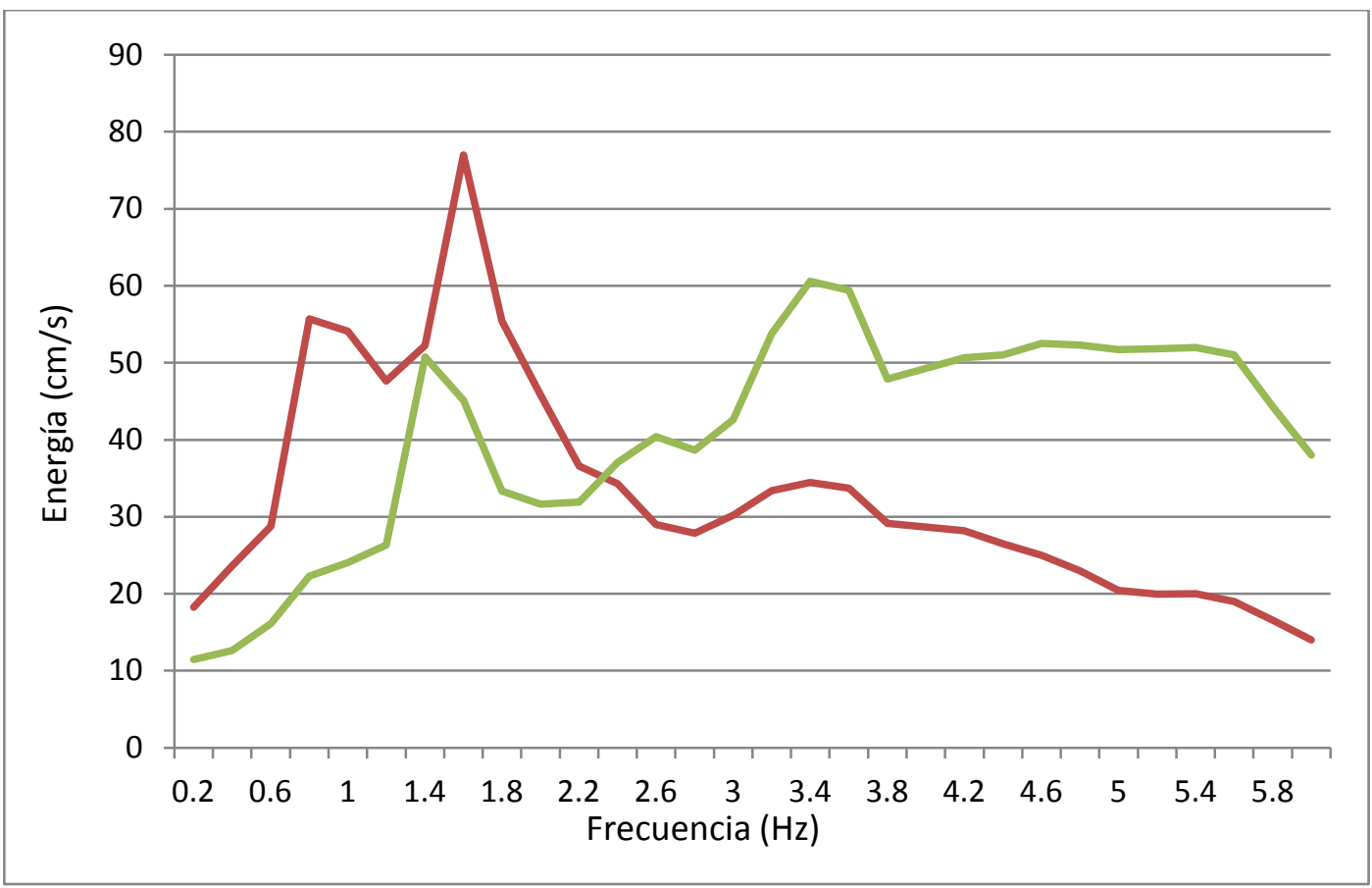

Figura 7.16a. Espectros de input de energía obtenidos antes de aplicar la función de amplificación

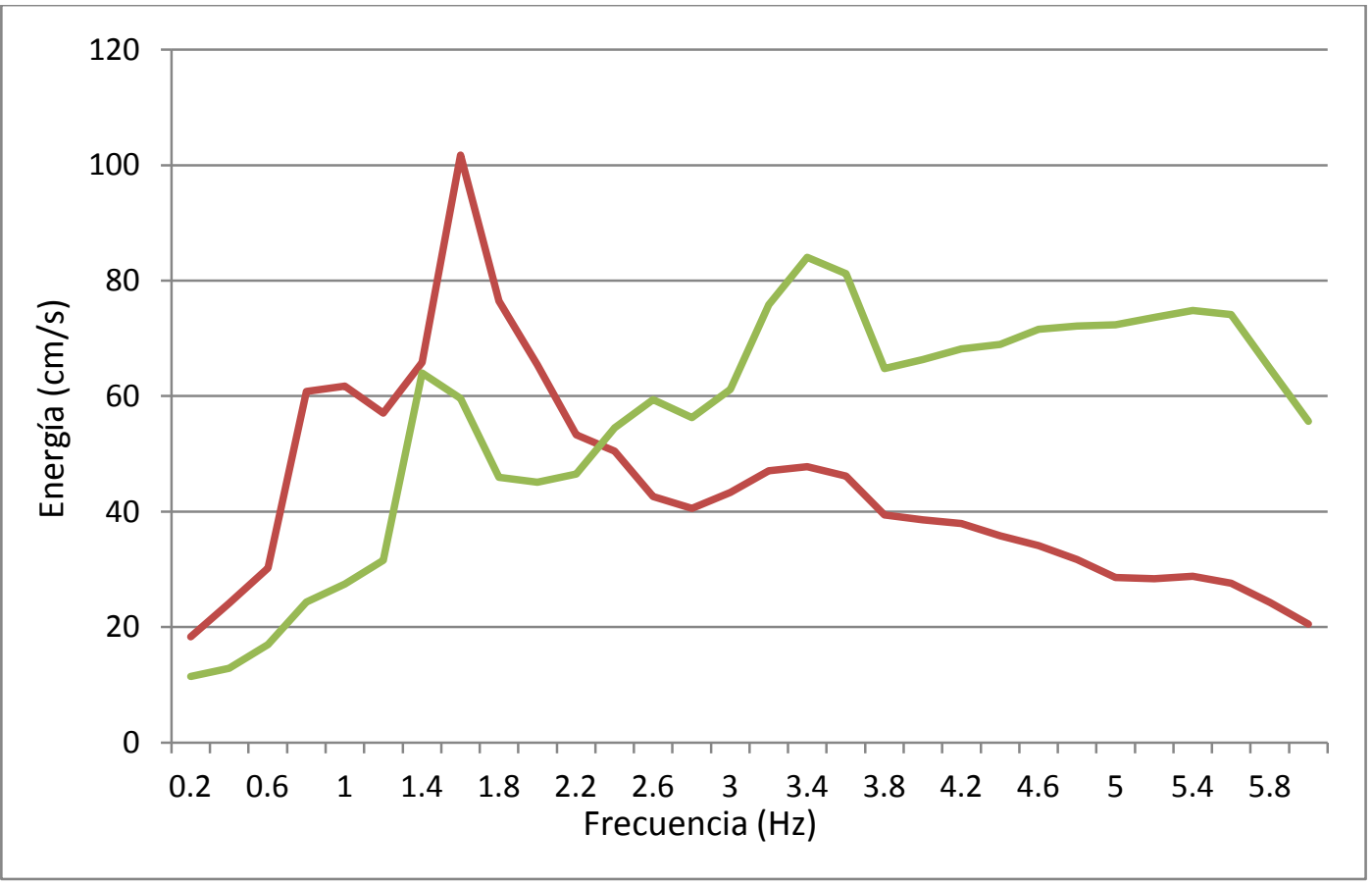

Figura 7.16b. Espectros de input de energía obtenidos tras aplicar la función de amplificación 


\section{Chana}

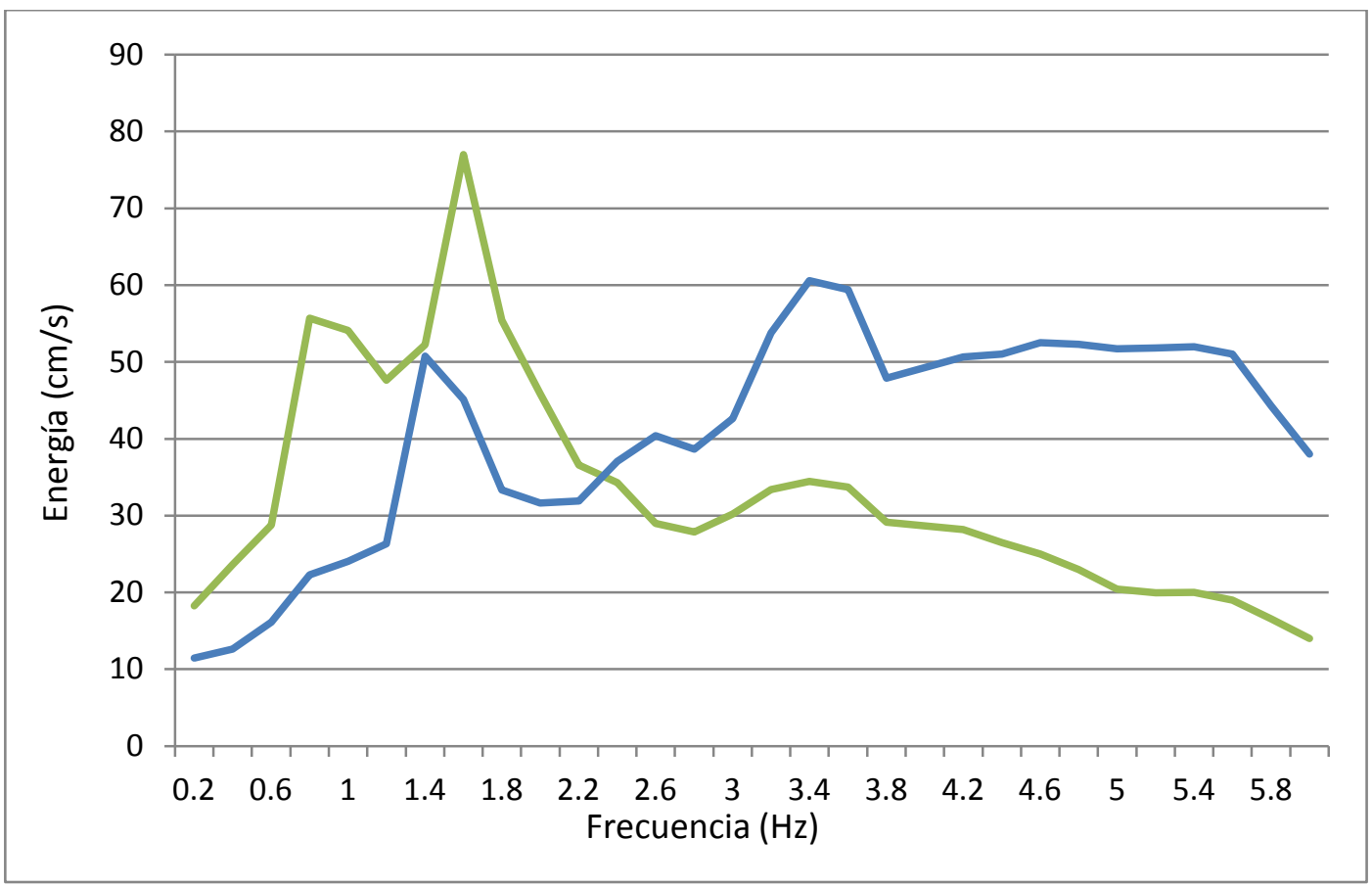

Figura 7.17a. Espectros de input de energía obtenidos antes de aplicar la función de amplificación

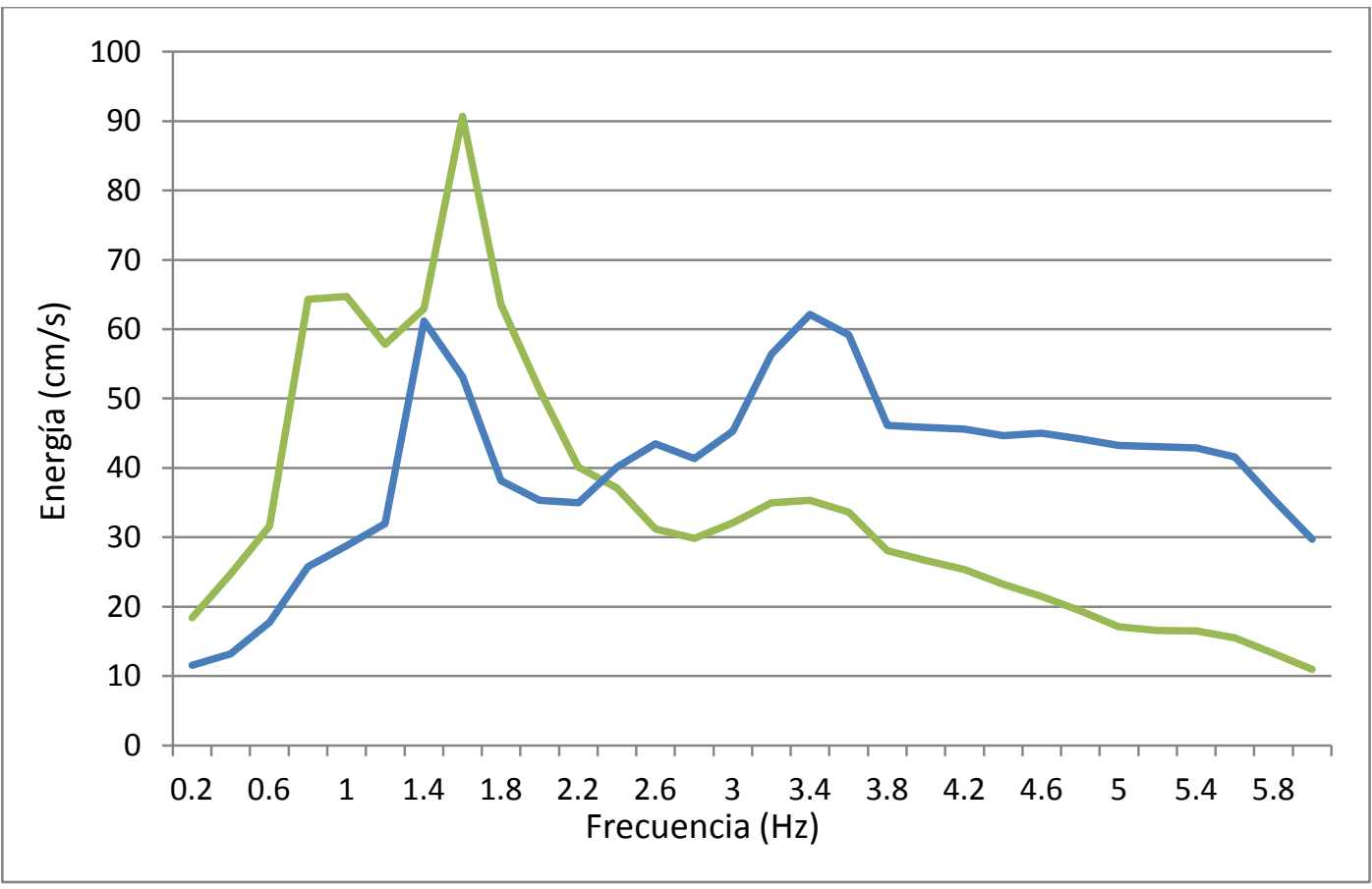

Figura 7.17b. Espectros de input de energía obtenidos tras aplicar la función de amplificación 


\section{Estadio de la Juventud}

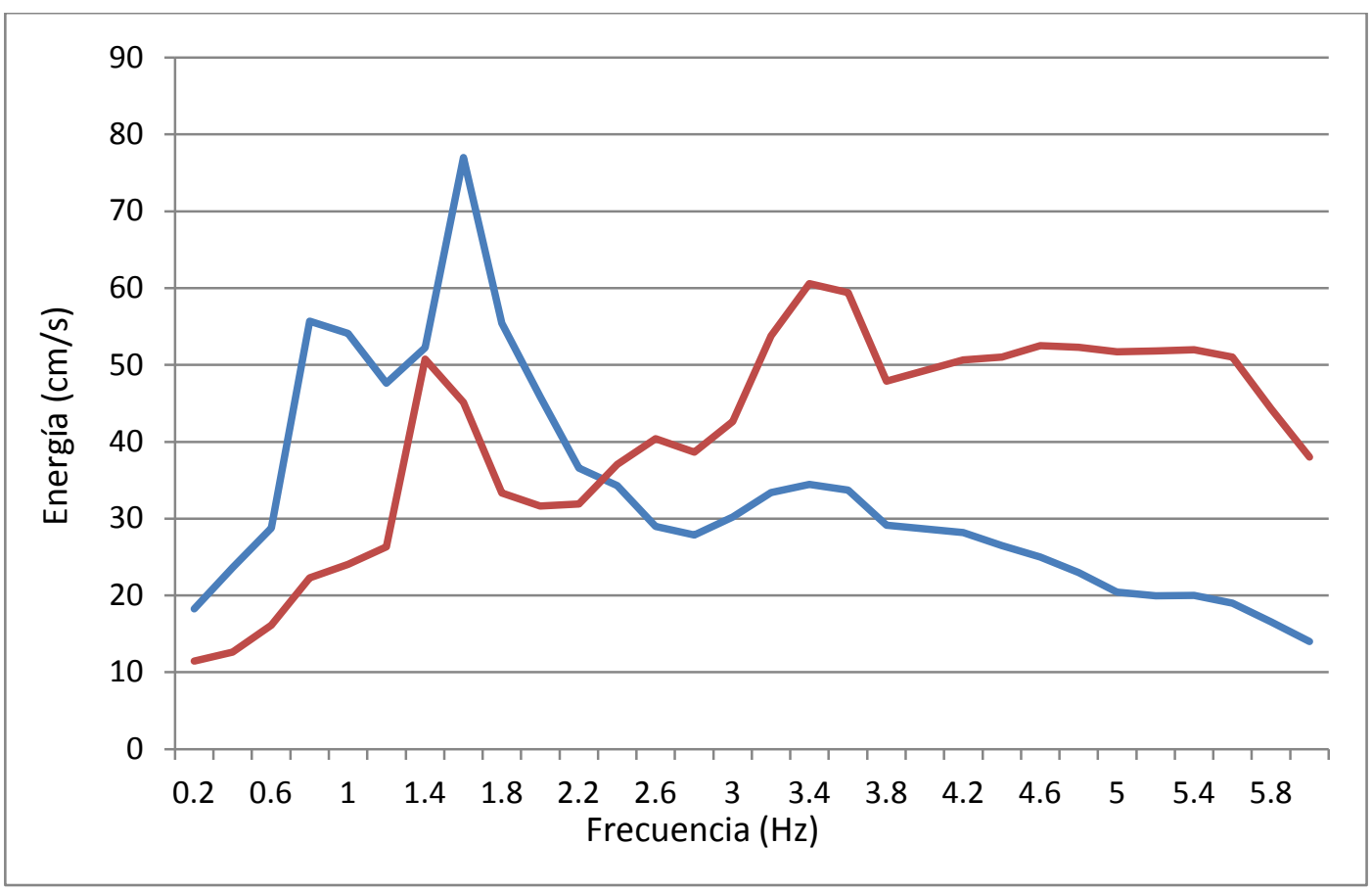

Figura 7.18a. Espectros de input de energía obtenidos antes de aplicar la función de amplificación

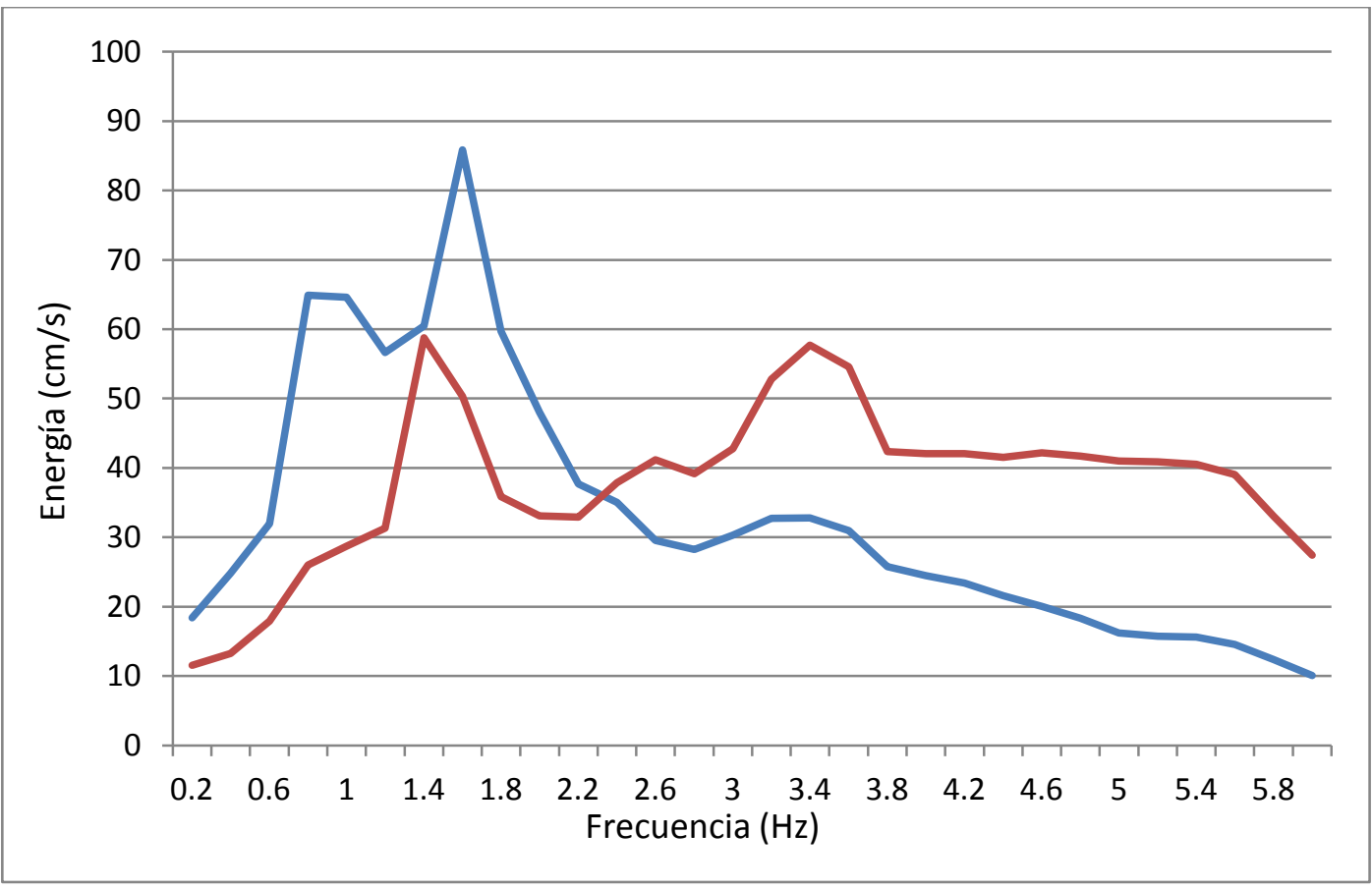

Figura 7.18b. Espectros de input de energía obtenidos tras aplicar la función de amplificación 


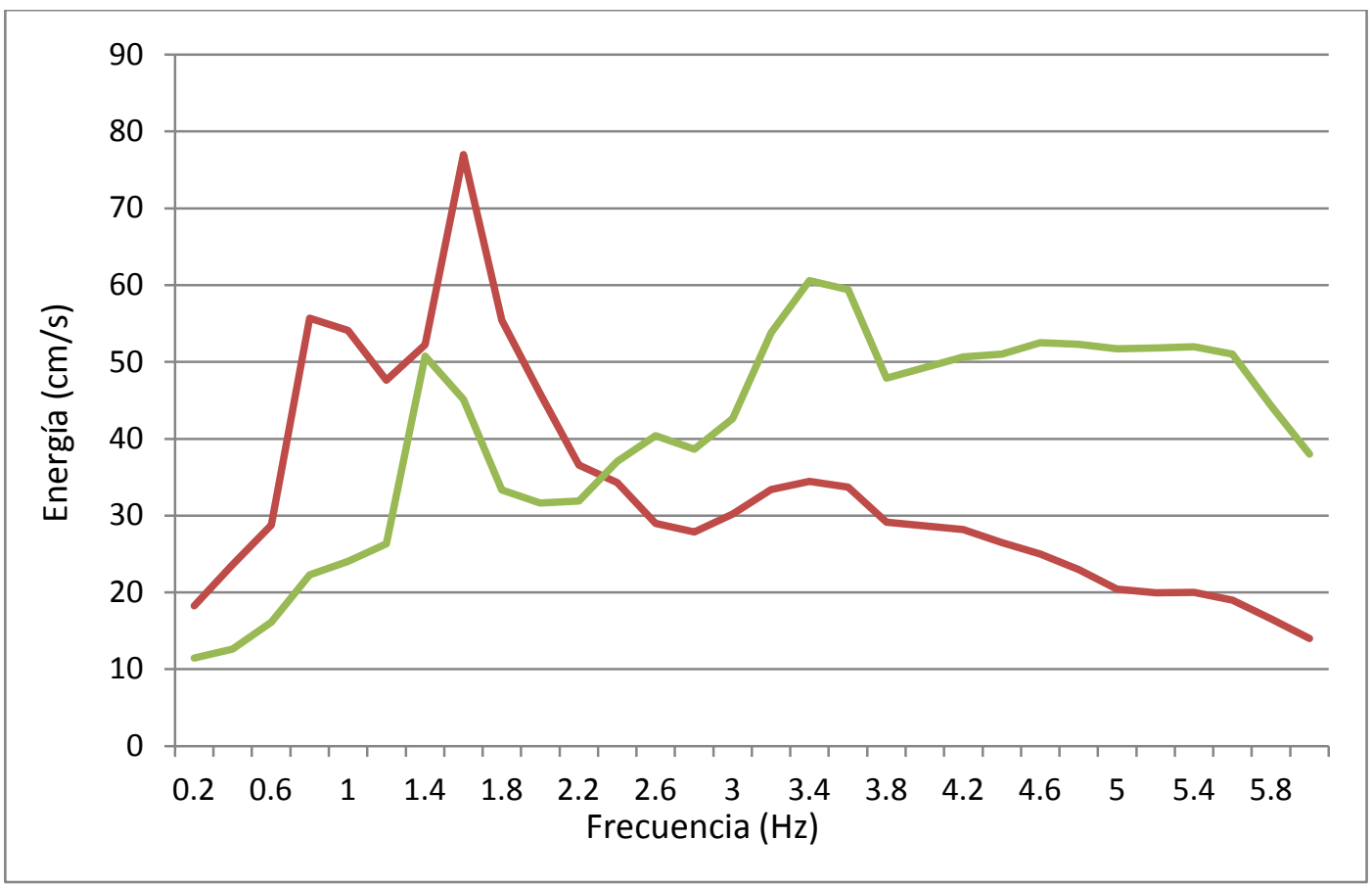

Figura 7.19a. Espectros de input de energía obtenidos antes de aplicar la función de amplificación

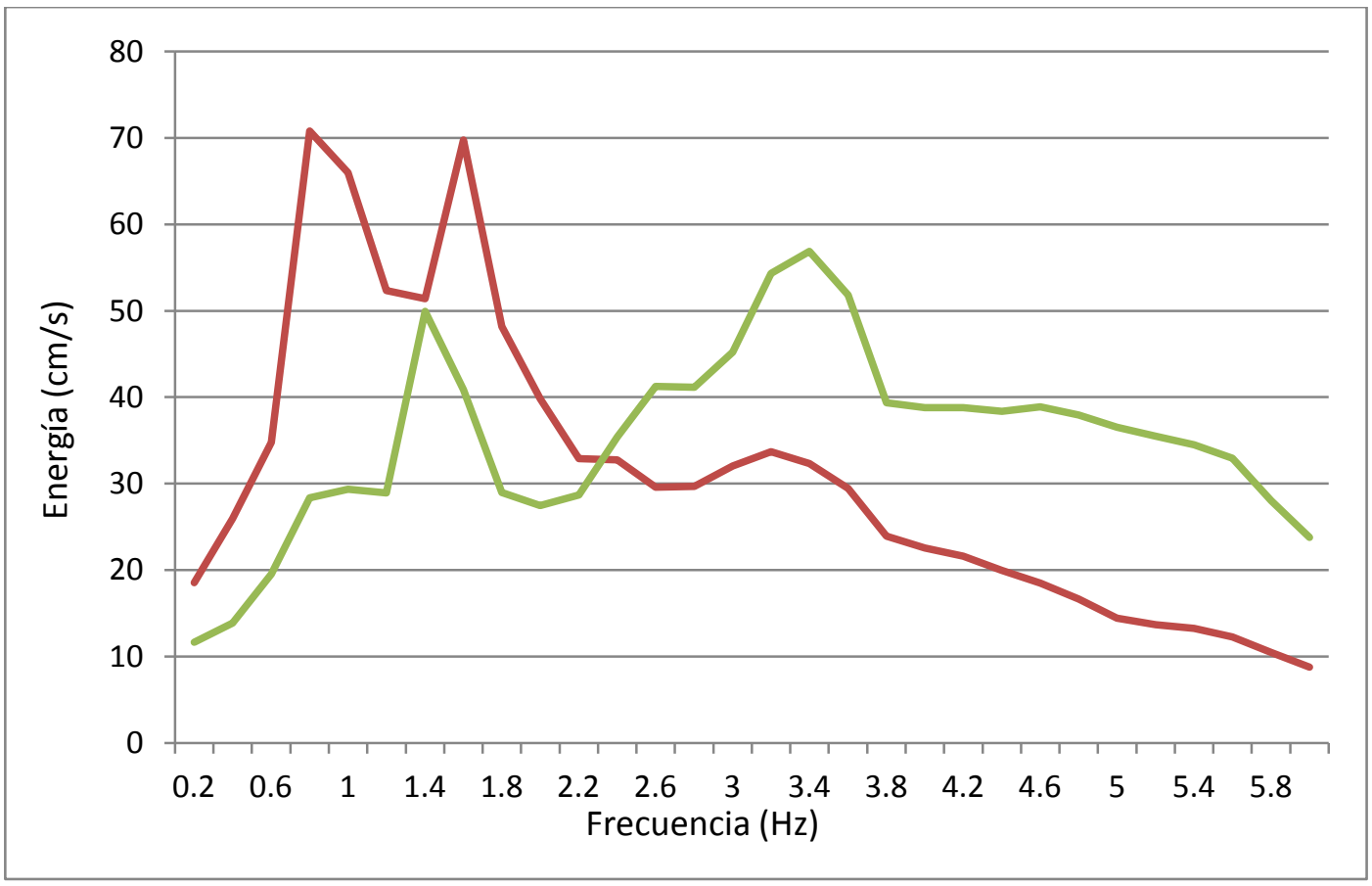

Figura 7.19b. Espectros de input de energía obtenidos tras aplicar la función de amplificación 


\section{Fuentenueva}

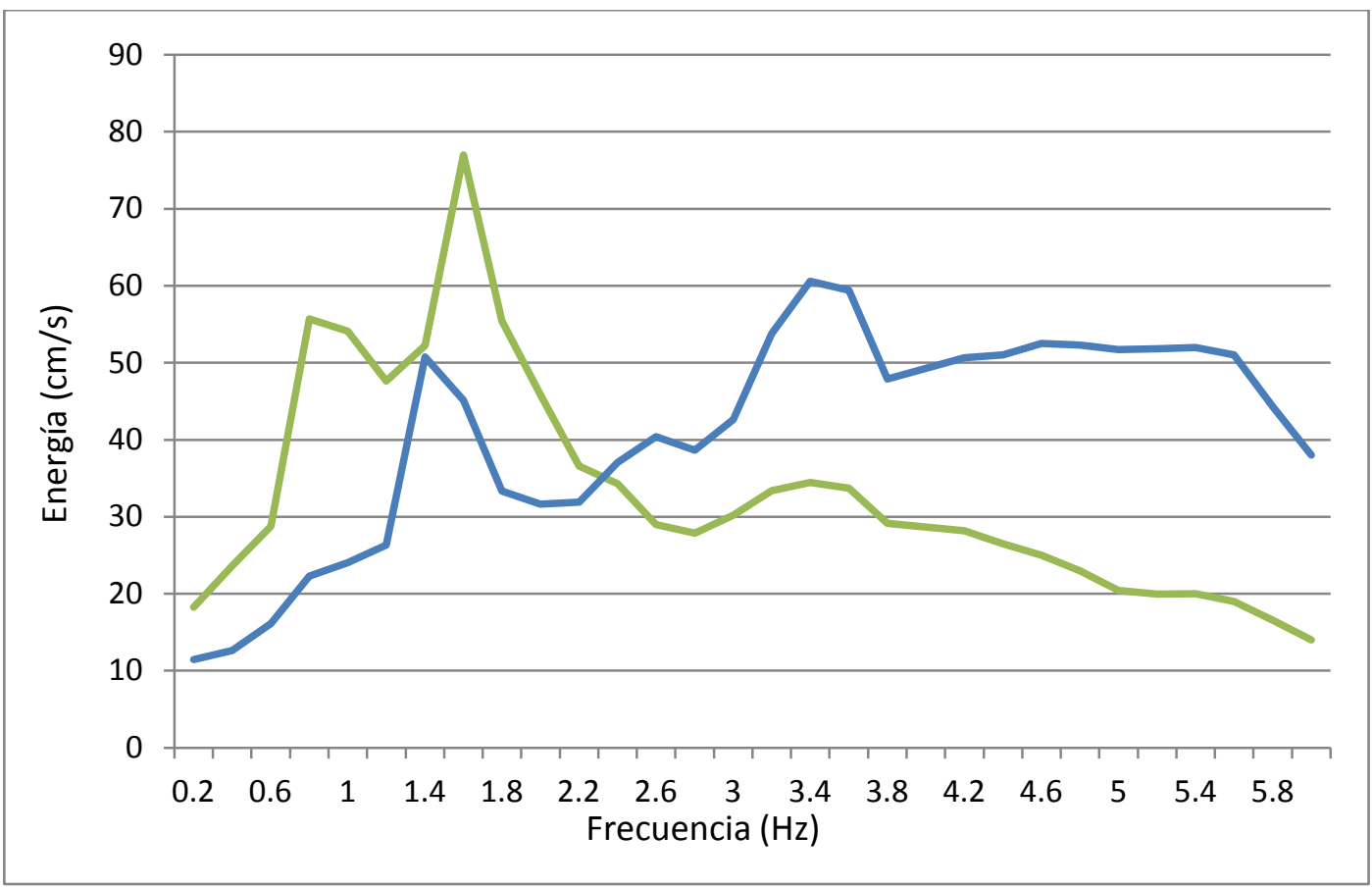

Figura 7.20a. Espectros de input de energía obtenidos antes de aplicar la función de amplificación

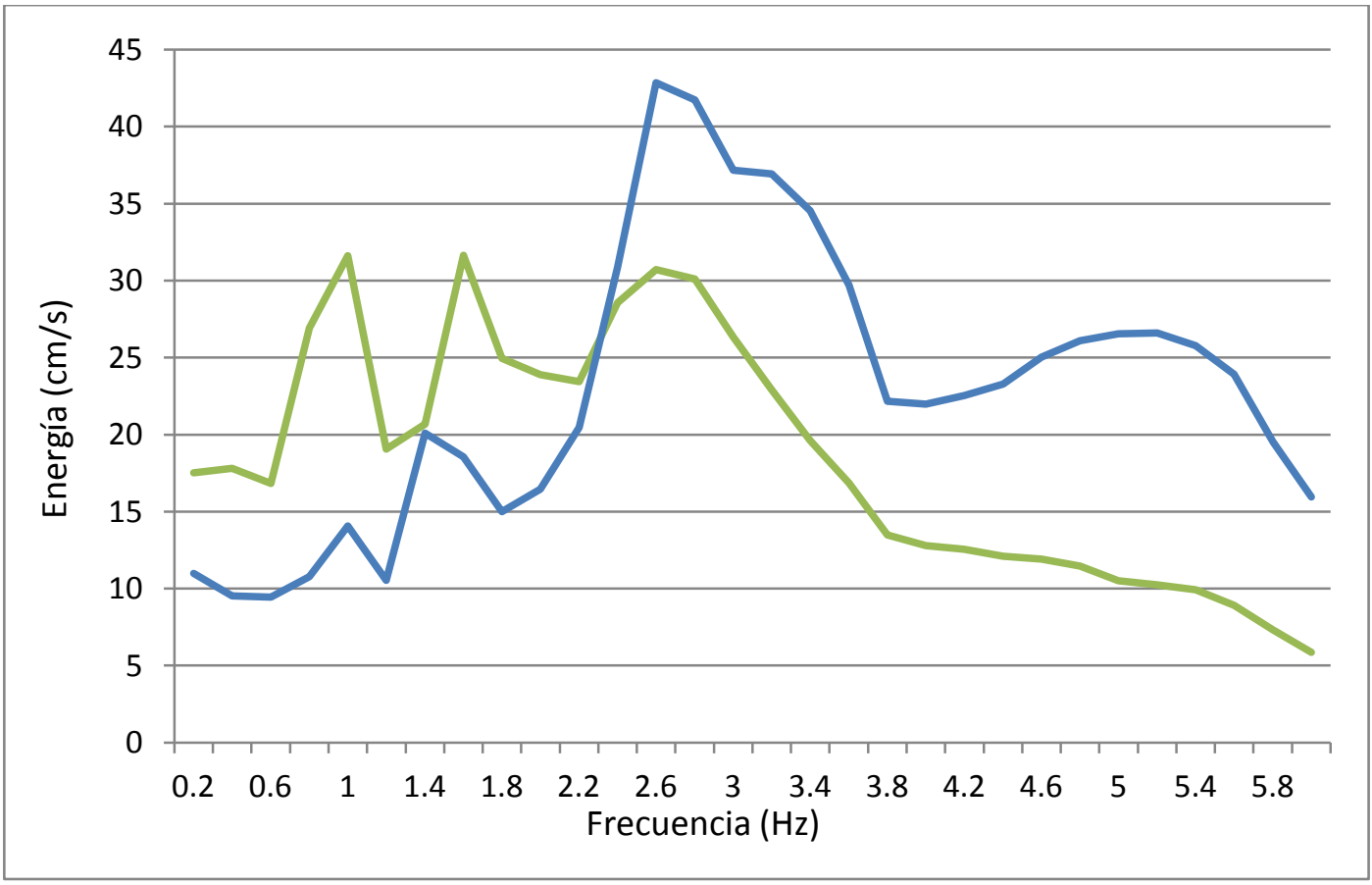

Figura 7.20b. Espectros de input de energía obtenidos tras aplicar la función de amplificación 
Ciudad Deportiva Granada 74

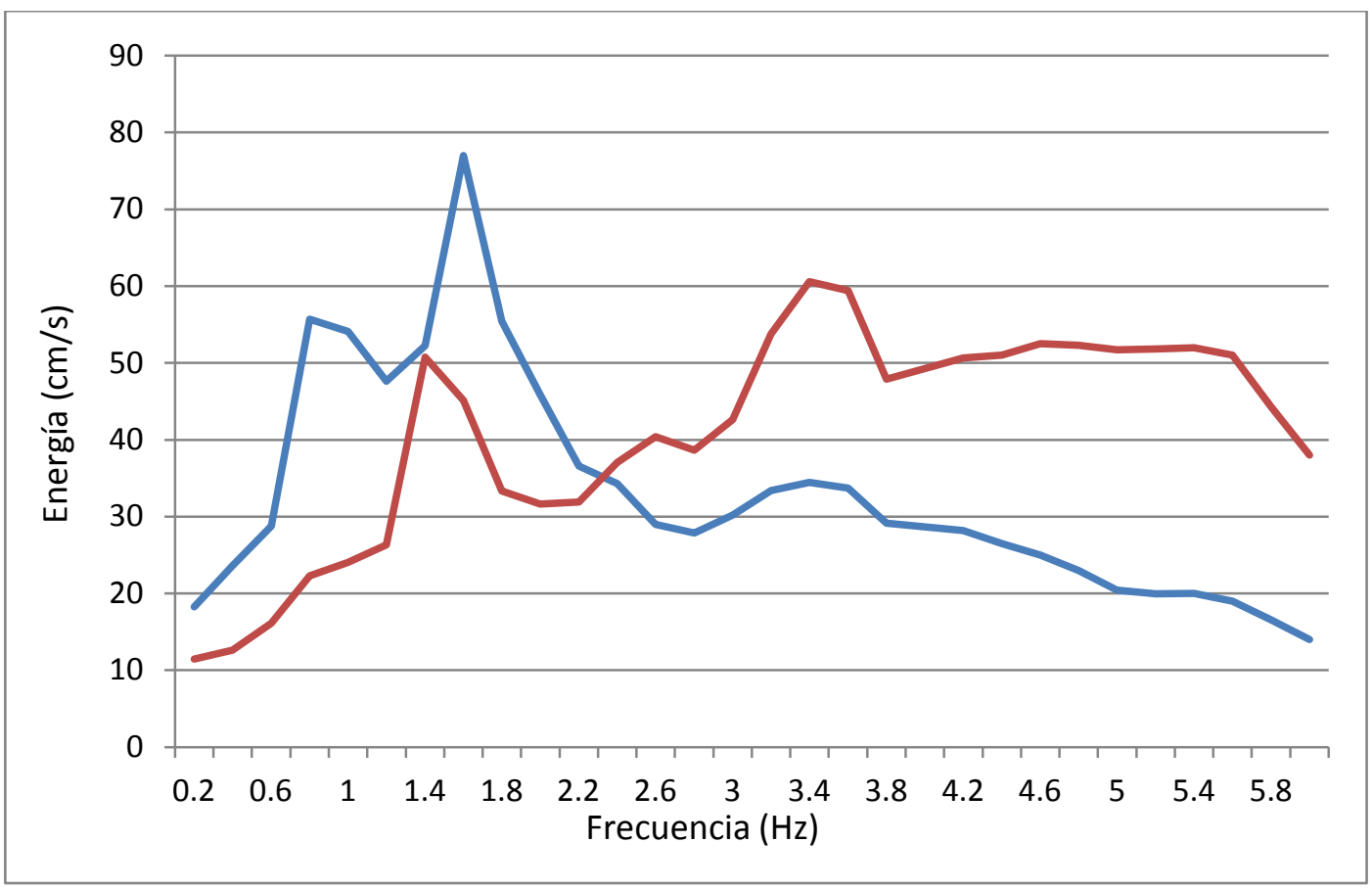

Figura 7.21a. Espectros de input de energía obtenidos antes de aplicar la función de amplificación

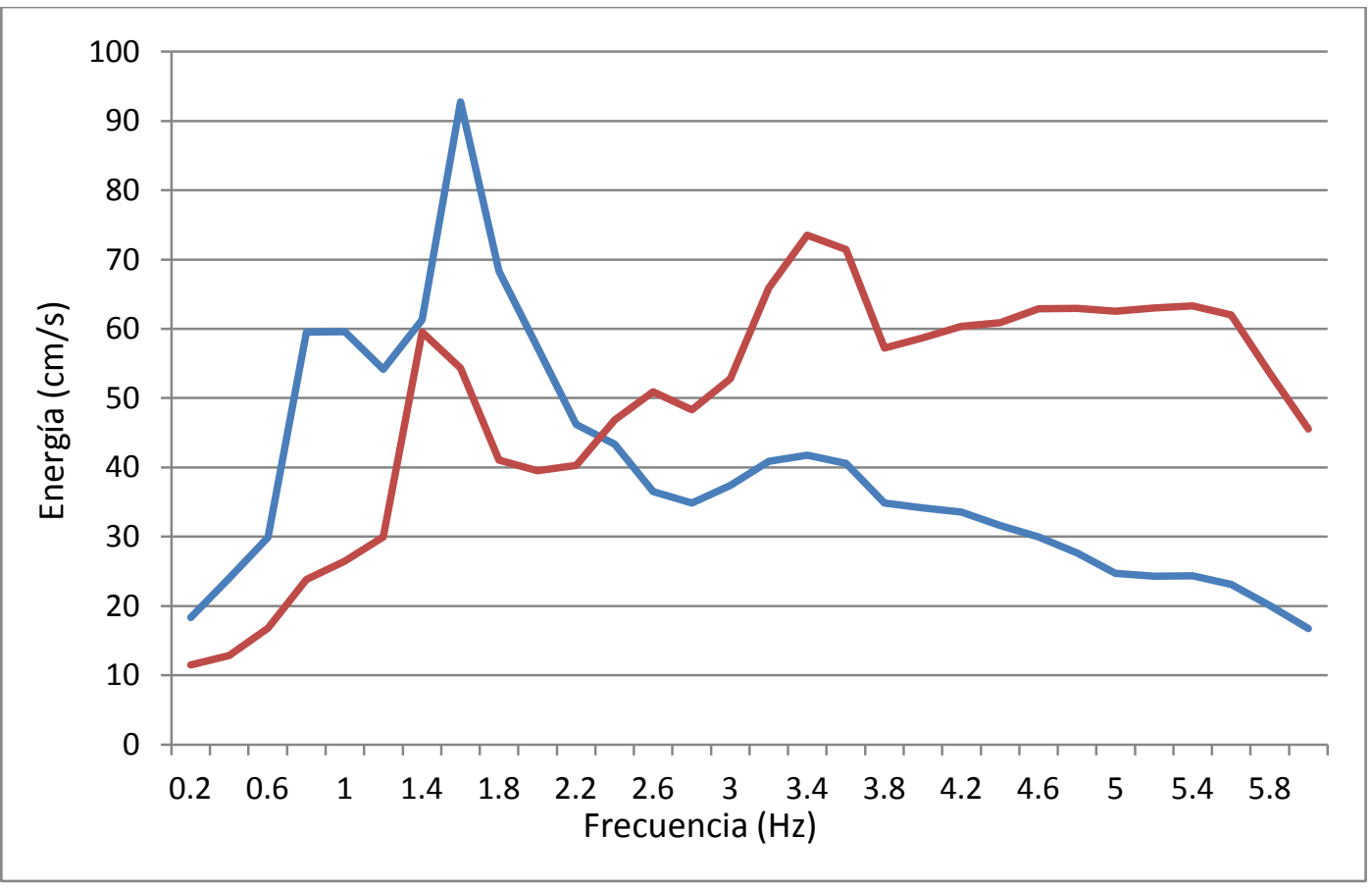

Figura 7.21b. Espectros de input de energía obtenidos tras aplicar la función de amplificació 


\section{Calle Náyades}

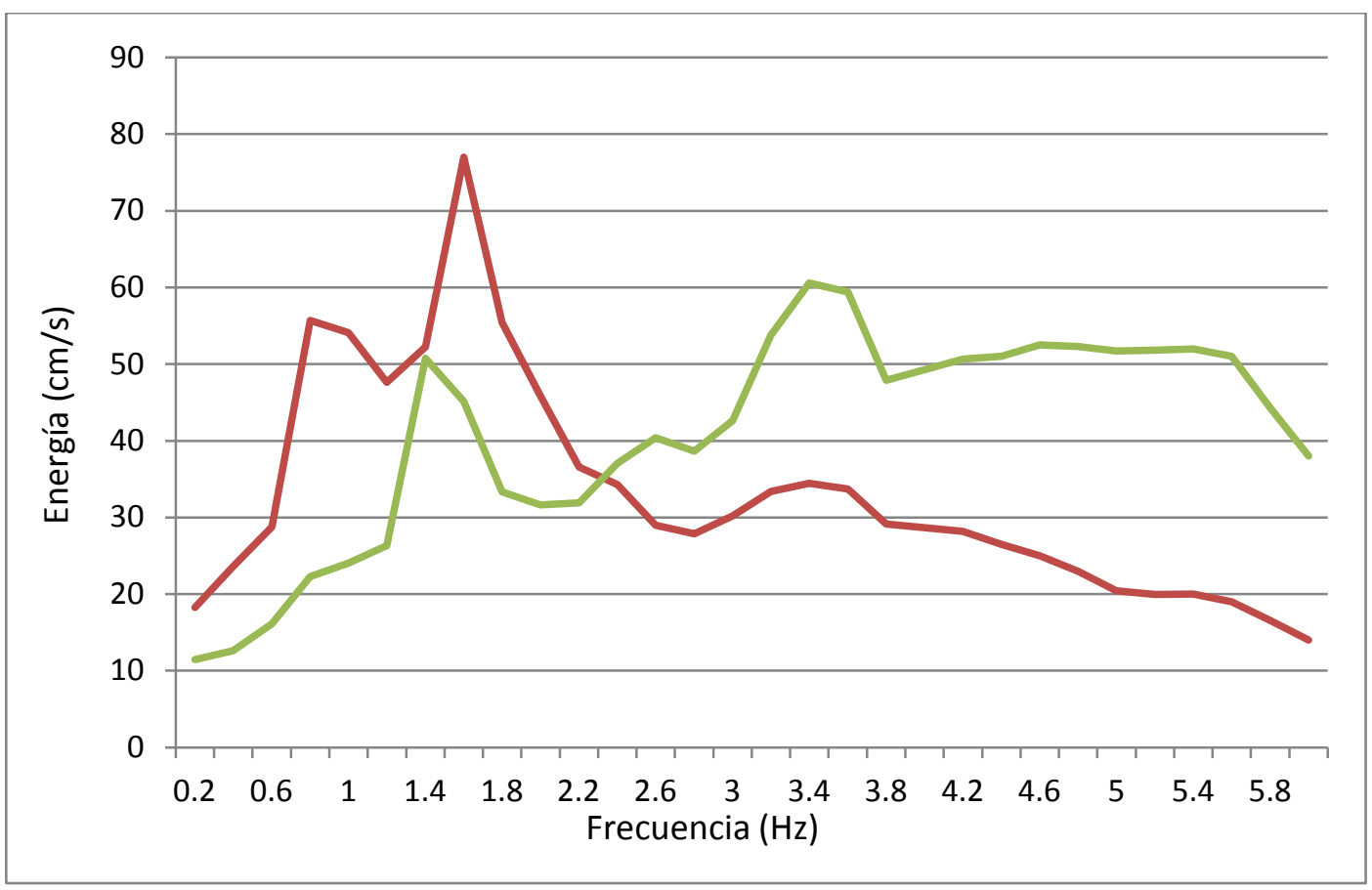

Figura 7.22a. Espectros de input de energía obtenidos antes de aplicar la función de amplificación

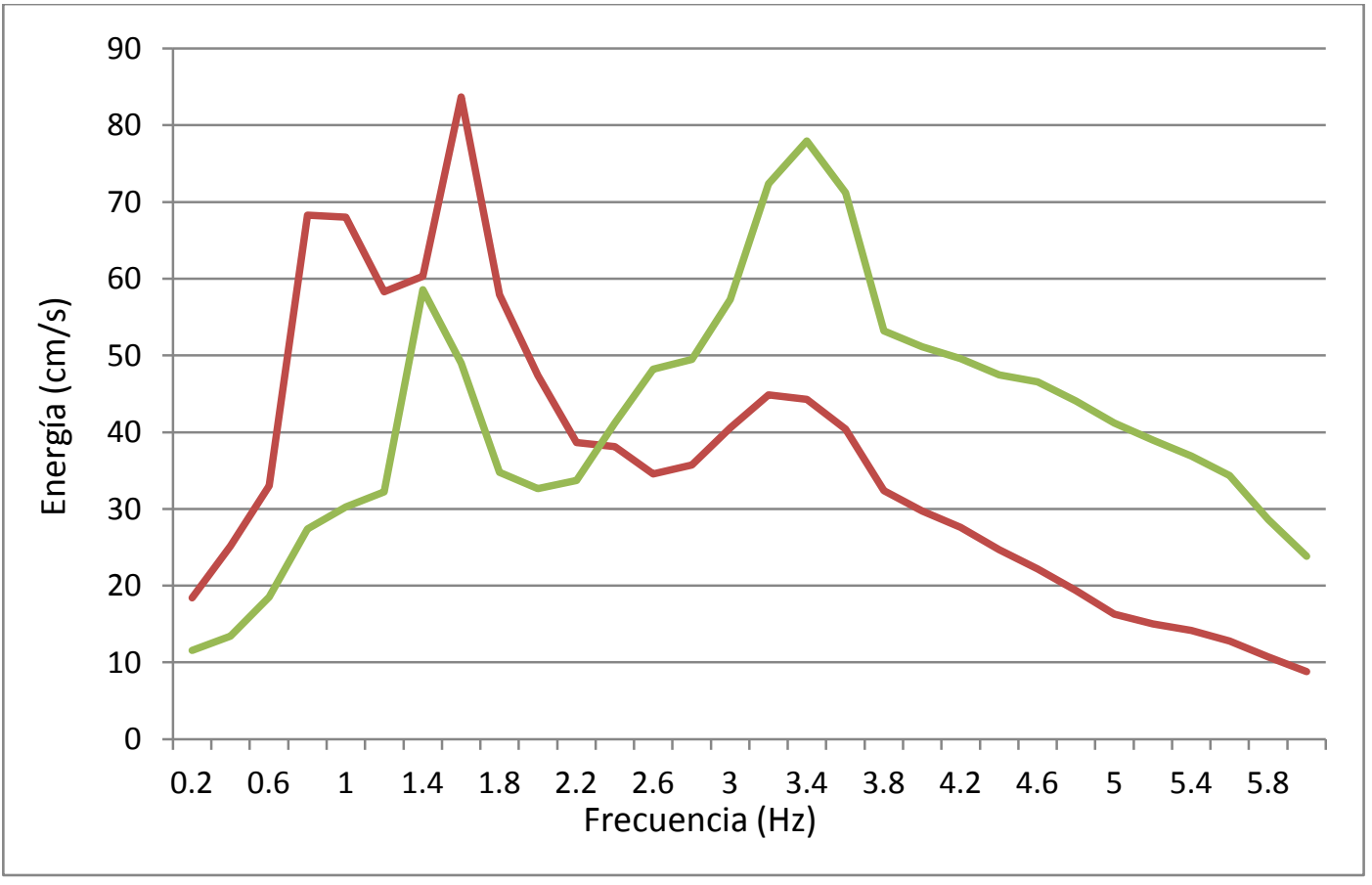

Figura 7.22b. Espectros de input de energía obtenidos tras aplicar la función de amplificación 


\section{Parque de las ciencias}

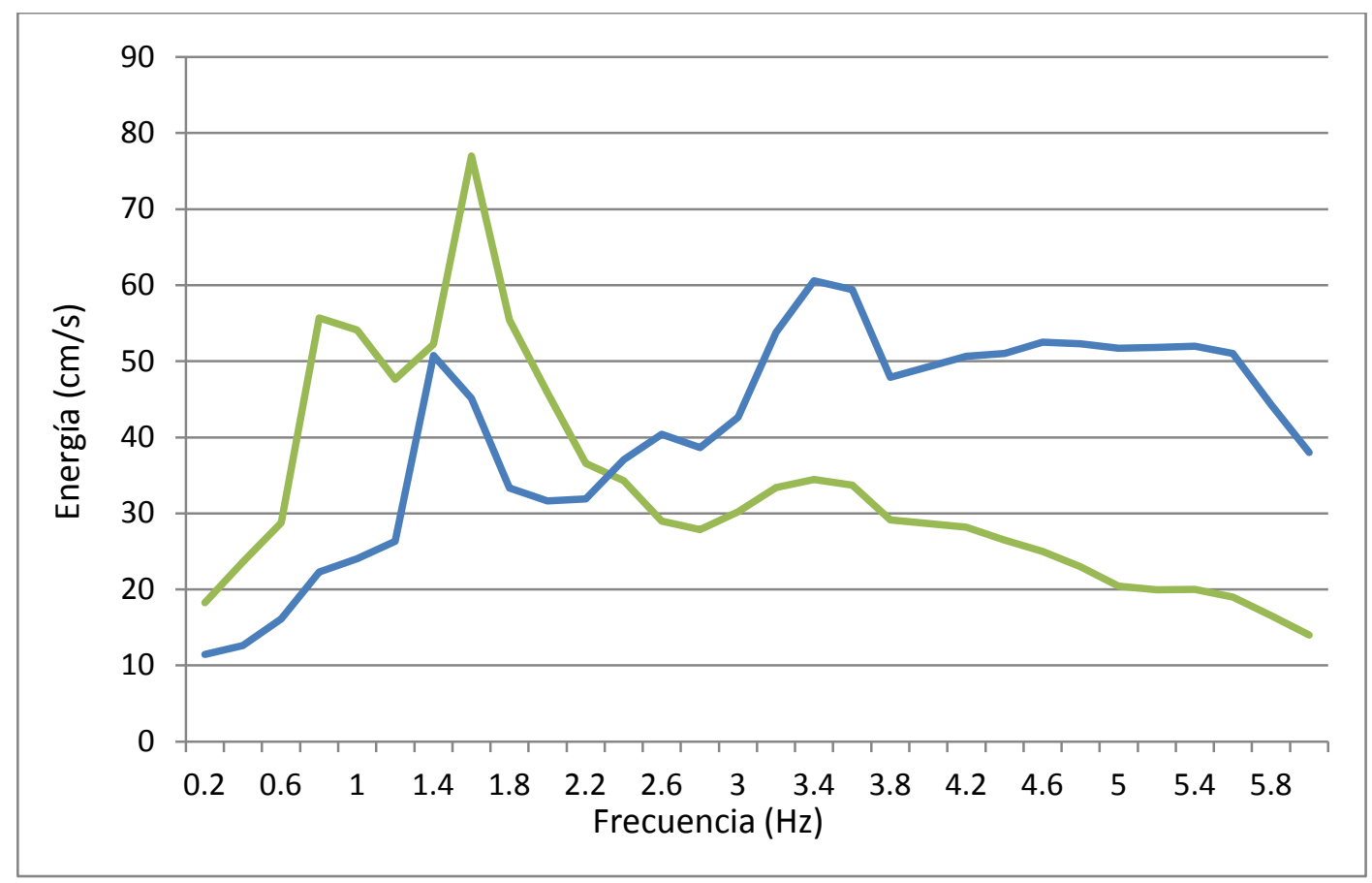

Figura 7.23a. Espectros de input de energía obtenidos antes de aplicar la función de amplificación

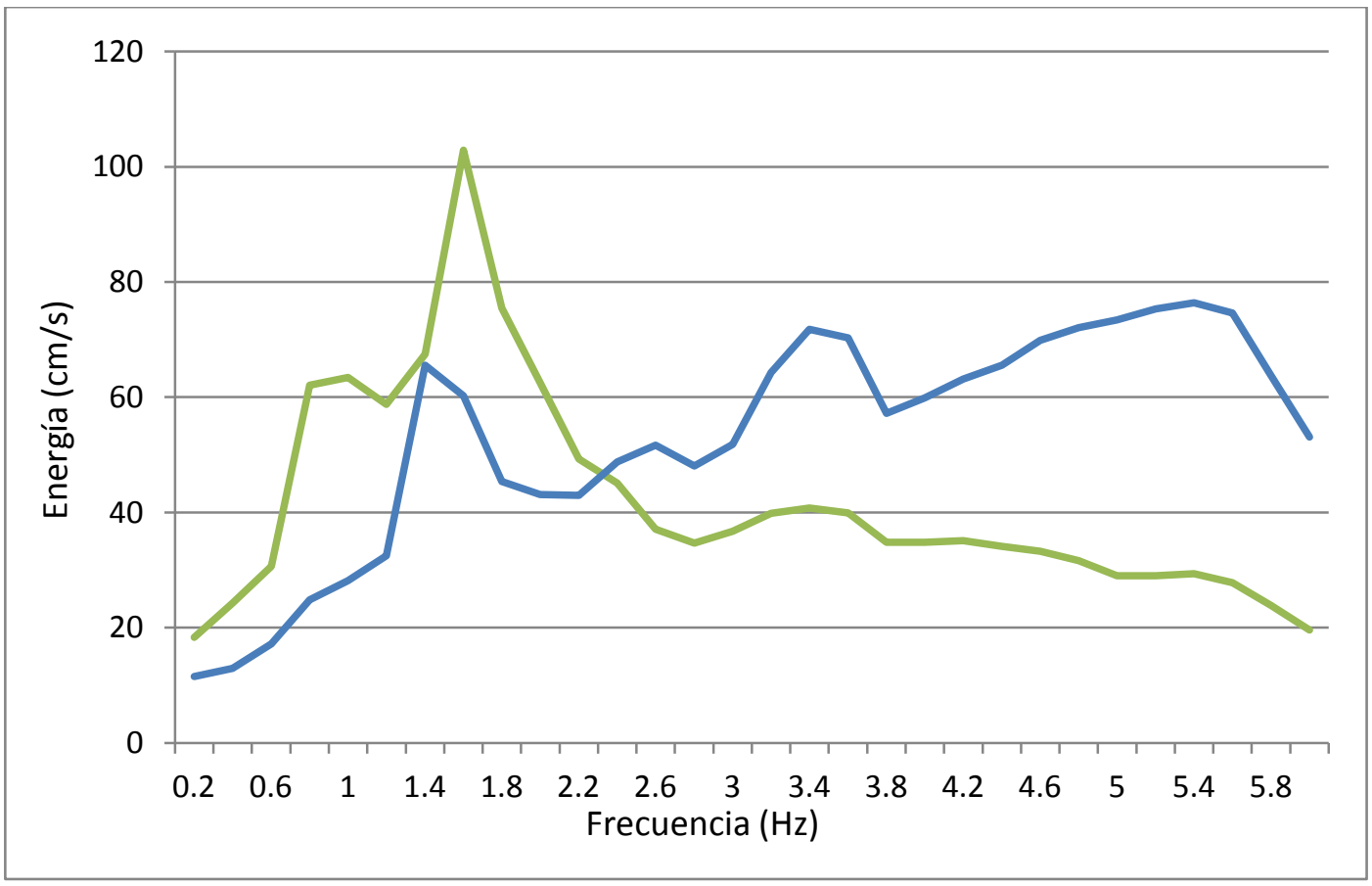

Figura 7.23b. Espectros de input de energía obtenidos tras aplicar la función de amplificación 


\section{Antigua fábrica de Sanders}

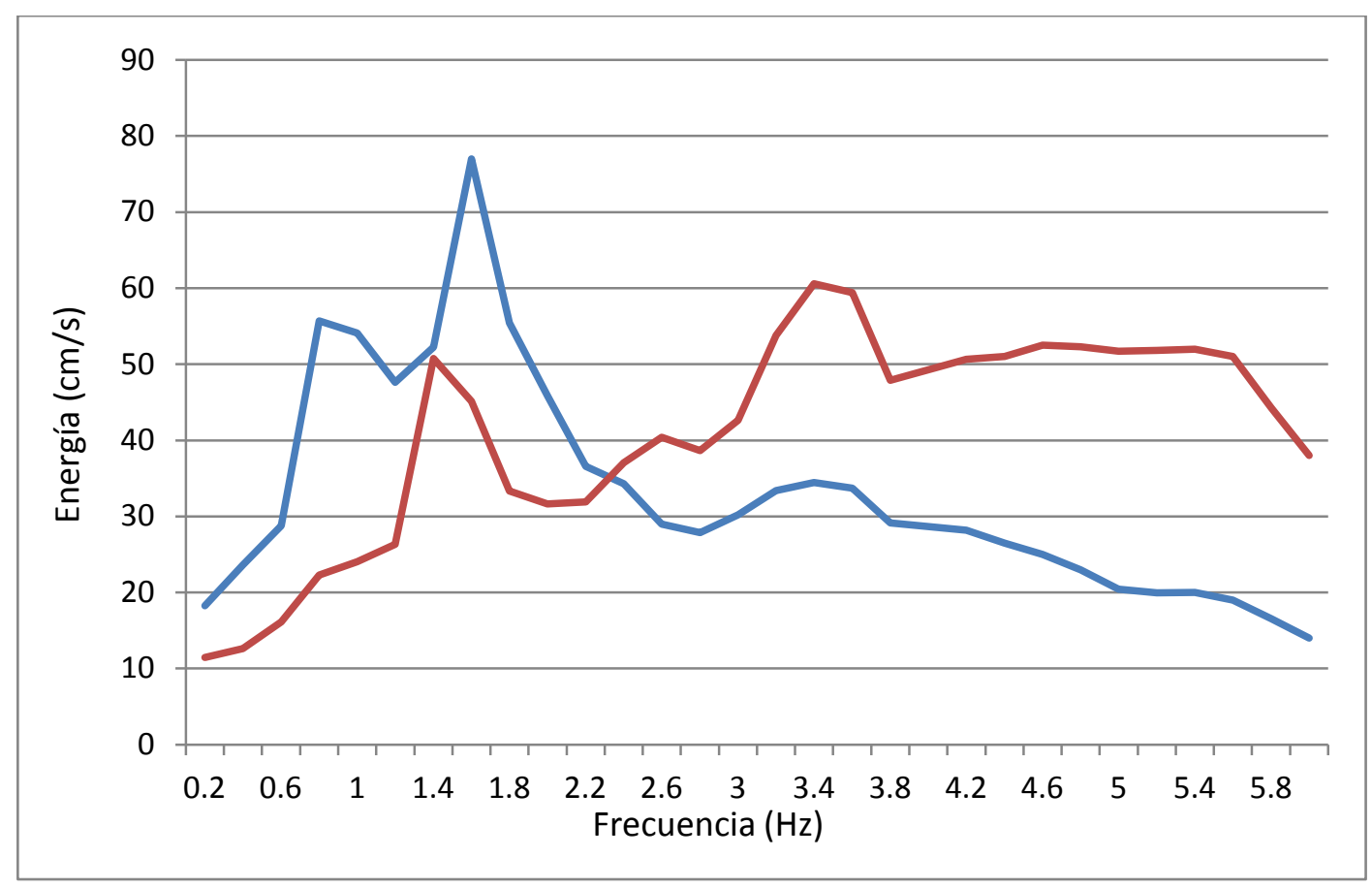

Figura 7.24a Espectros de input de energía obtenidos antes de aplicar la función de amplificación

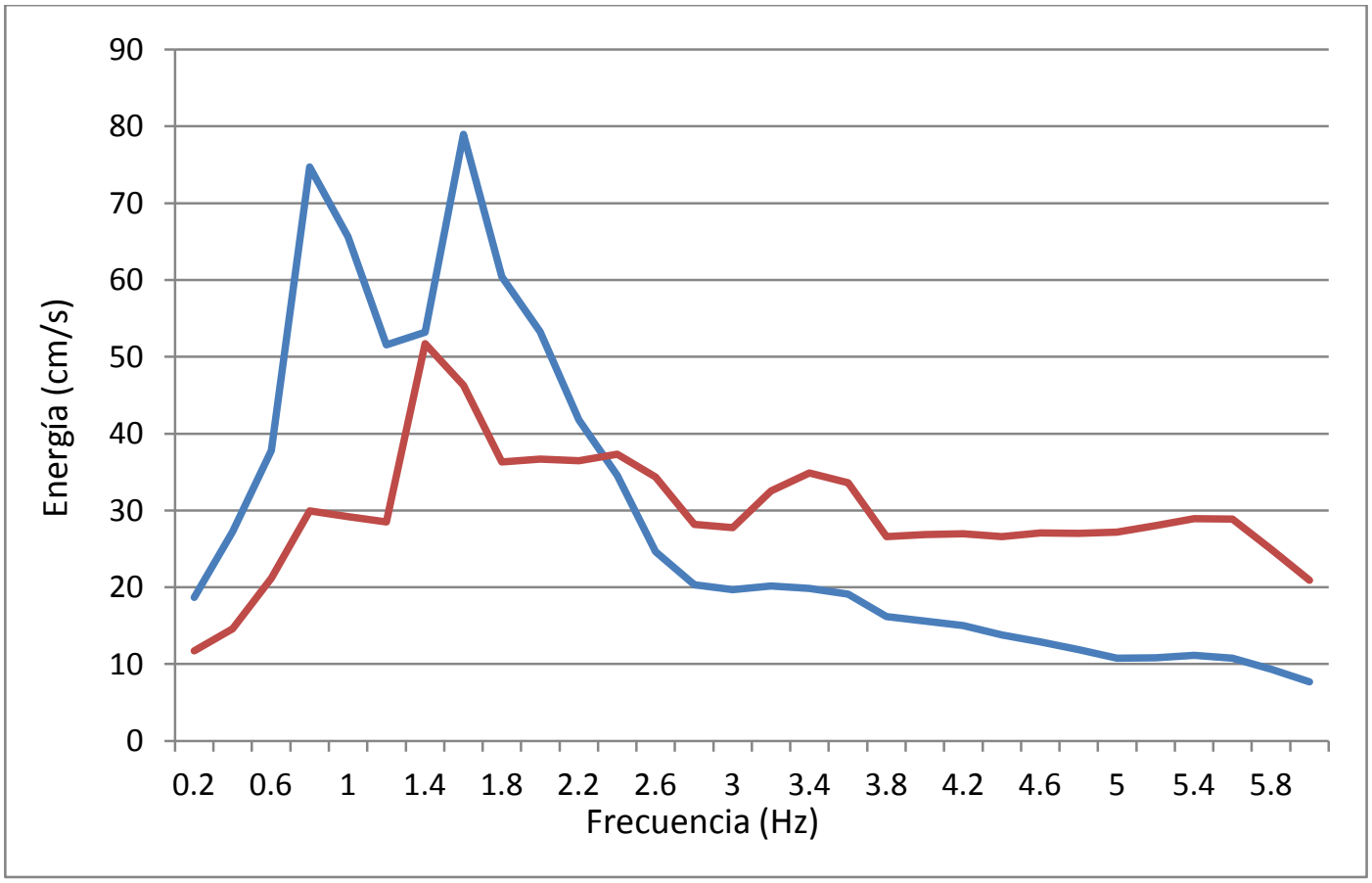

Figura 7.24b. Espectros de input de energía obtenidos tras aplicar la función de amplificación 
Vega de Granada

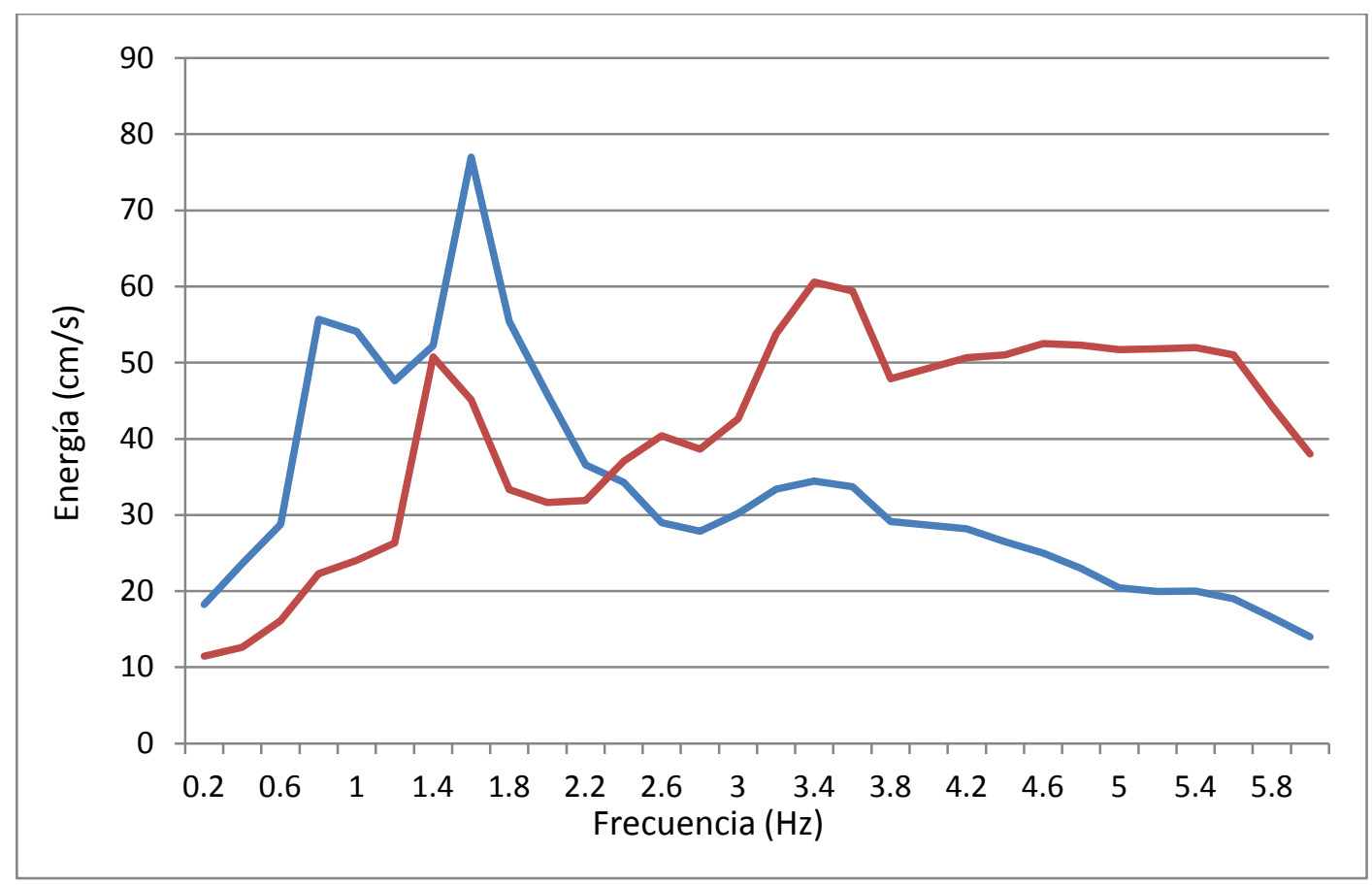

Figura 7.25a Input de energía obtenidos antes de aplicar la función de amplificación

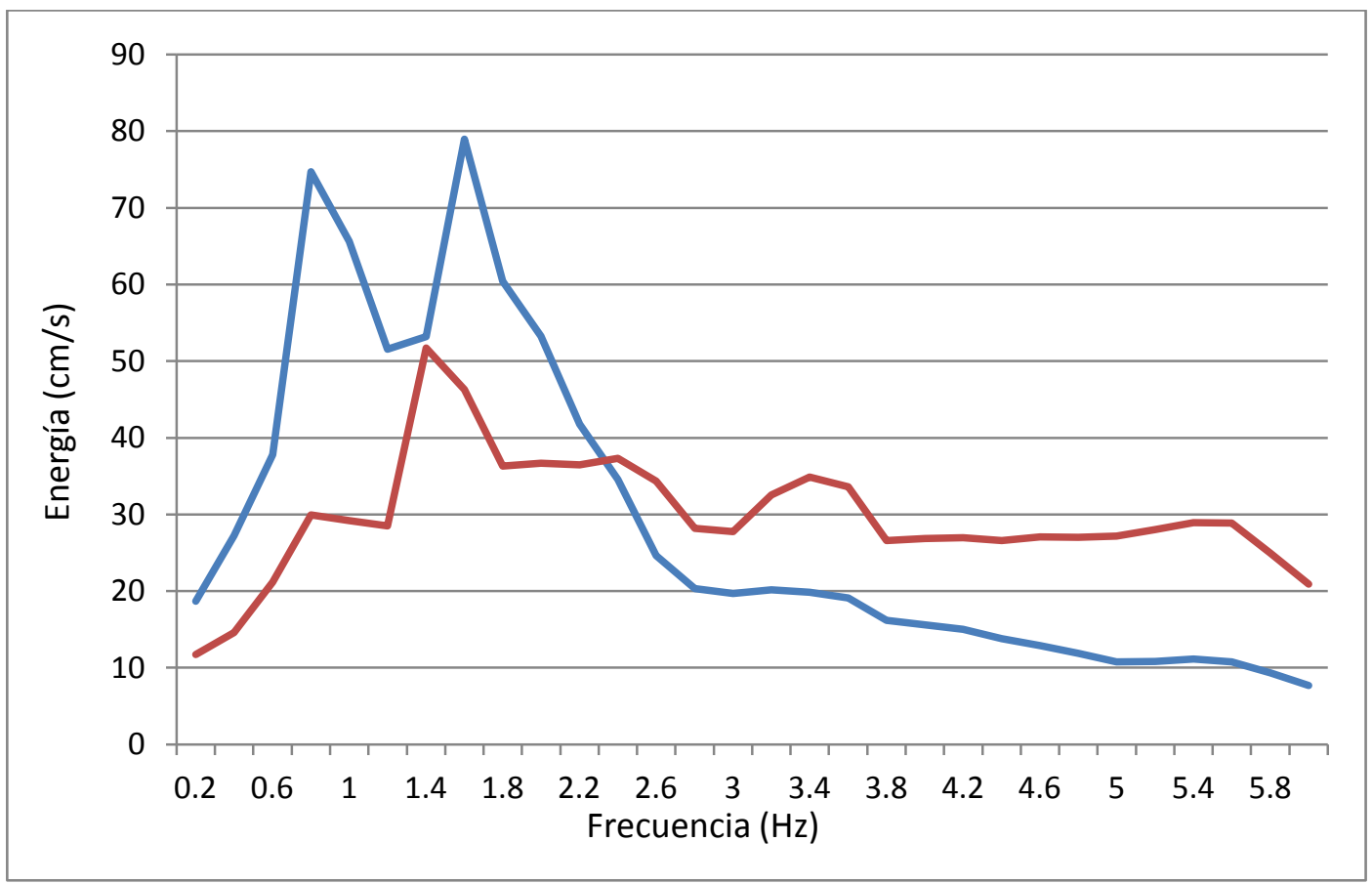

Figura 7.25b. Espectros de input de energía obtenidos tras aplicar la función de amplificación 


\section{Zaidín}

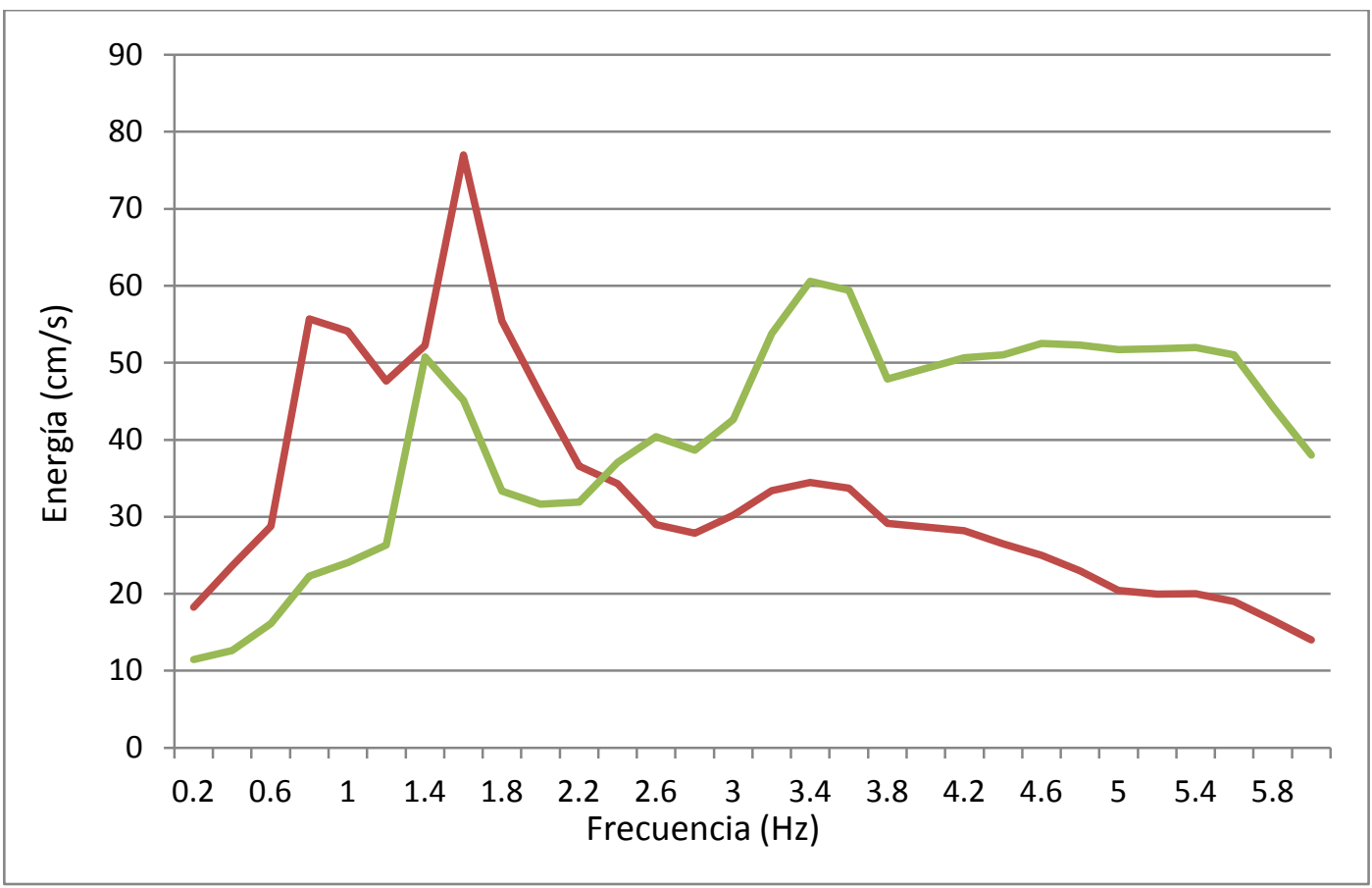

Figura 7.26a Espectros de input de energía obtenidos antes de aplicar la función de amplificación

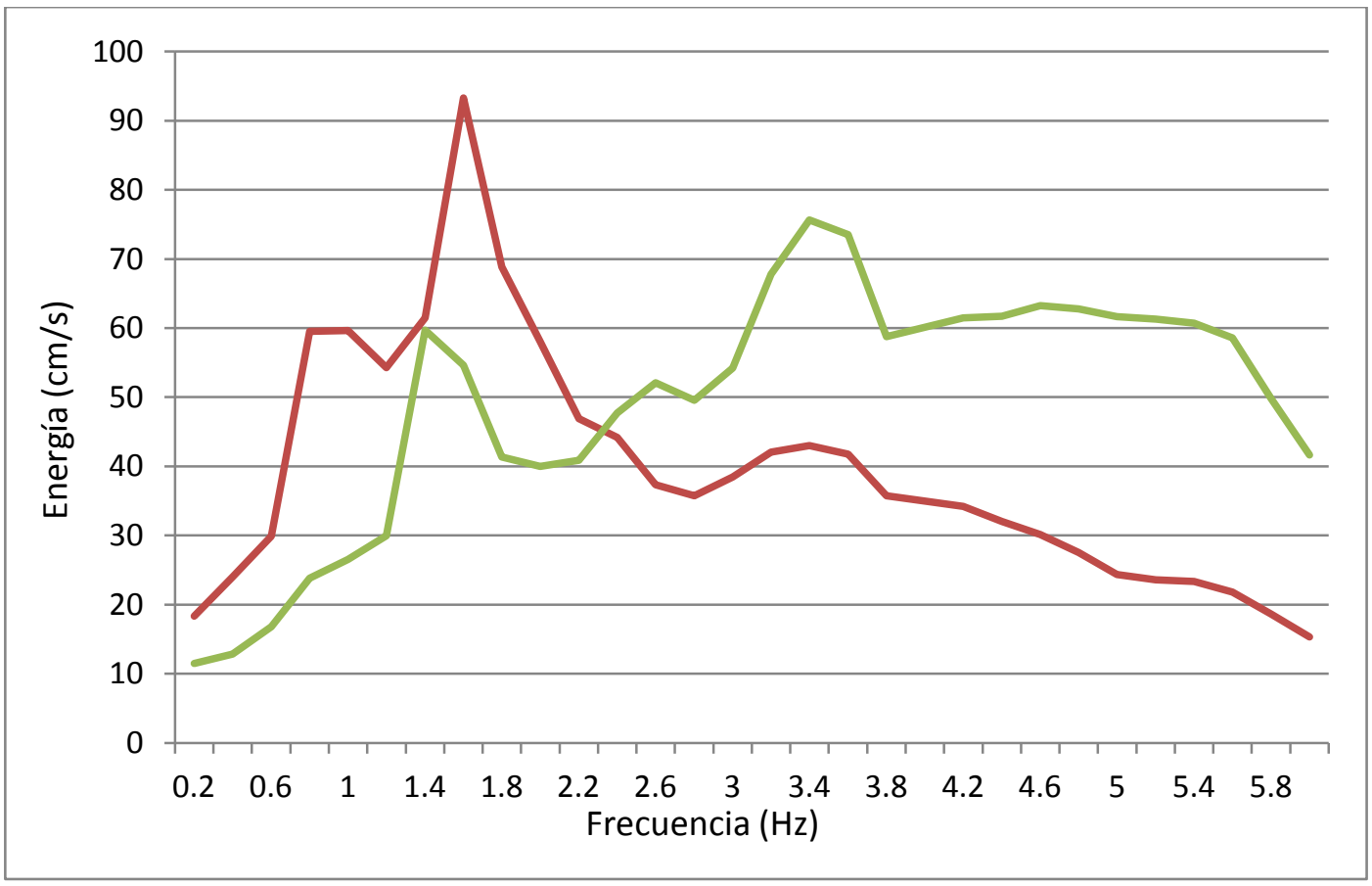

Figura 7.26b. Espectros de input de energía obtenidos tras aplicar la función de amplificación 


\section{DISCUSIÓN GENERAL DE RESULTADOS}

Durante el presente trabajo se ha visto y comprobado como la respuesta local del terreno modifica el input sísmico de las capas profundas, lo que hace que sea necesaria una modelización del terreno y del comportamiento sísmico para poder tener una idea de cómo será el movimiento en superficie.

El método de Nakamura resulta adecuado con el objetivo de identificar el periodo dominante, pero su uso extensivo para averiguar las amplificaciones del terreno debido a la respuesta local es, como se ha indicado, discutible. Esto puede deberse a que dicho método funciona bien cuando hay un contraste de impedancias fuerte entre las diferentes capas del modelo, pero no en caso contrario.

A través de la modelización llevada a cabo con la metodología analítica de Tsai (1969) se ha conseguido obtener una función de transferencia que relaciona los input sísmicos en capas de basamento con los inputs en la superficie, tras ser modificado por su propagación a través de un modelo de capas.

En el caso de la modelización por el modelo analítico de Tsai (1989) la influencia del factor de amortiguamiento es crucial a la hora de obtener unos datos válidos. Se han intentado modelizar con diferentes coeficientes de amortiguamiento utilizándose finalmente un valor igual al 5\%. Se ha observado que cualquier variación de éste afecta notablemente al resultado de la función de transferencia.

Una de las ventajas observadas en el uso del método de Tsai (1969) respecto del de Nakamura (1989) es que, al ser un método analítico contra uno empírico, se pueden observar claramente la influencia de los diferentes factores que forman los modelos.

Las estructuras locales estudiadas se limitan a las capas más superficiales, por la pequeña apertura de las antenas utilizadas en los experimentos de SPAC para este caso. Esto hace que sólo se vean afectadas, por los modelos de capas correspondientes, las componentes espectrales de frecuencias relativamente altas o periodos cortos. Sin embargo, la estructura local más profunda podría modificar las características en otros periodos más largos. 


\section{CONCLUSIONES}

Se han implementado dos métodos diferentes, uno analítico y otro semiempírico, comparándose los resultados de la función de transferencia obtenida para ambos.

Se observa como el método de Nakamura (1989) nos da información sobre los periodos dominantes. Para los suelos estudiados dichos periodos resultan bastante cortos, entre 0,2 y 1 segundos.

Los valores máximos de la amplificación calculados con la técnica de Tsai , usando los modelos de capas obtenidos con SPAC, oscilan entre valores de 1,1 y 1,7 , dependiendo del suelo en estudio. Estos valores son menores de los que se podían esperar a priori.

Las zonas de mayor amplificación corresponden a los puntos de estudio localizados en el Zaidín y en el Parque de las Ciencias, con valores máximos calculados de 1,7 y 1,5 respectivamente. A su vez las zonas de menor amplificación se corresponden con el Campus de Fuentenueva y la zona del Estadio de la Juventud con valores de 1,1 y 1,2 .

Para las simulaciones llevadas a cabo, utilizando dos sismos italianos, se observa que los inputs de energía son modificados por el efecto de sitio, viéndose incrementados para algunos periodos determinados. Este tipo de resultados, tras ser comprobados con más registros, son los que se deberían tener en cuenta a la hora de plantear edificaciones con periodos propios cercanos a estos valores de máxima amplificación.

El valor del amortiguamiento utilizado afecta significativamente a los resultados de la respuesta local obtenida con la técnica de Tsai, por lo que sería necesario un mejor conocimiento del mismo. 


\section{REFERENCIAS}

Aguirre, " SPAC Method: Another alternative to estimate site effect in Mexico City". Instituto de Ingeniería UNAM.

Aki K (1957). Space and time spectra of stationary stochastic waves, with special reference to microtremors. Bulletin of the Earthquake Research Institute; 35: 415-456.

Borcherdt, R. D. (1994). "Estimates of site-dependent response spectra for design (methodology and justification) Earthquake Spectra", 10, 617-654.

CEMOSA S.A., (2005). Estudio Geotécnico: Guardería y comedor municipal en Los Mondragones (Granada).

Eurocode No.8 (1998). Design of structures for earthquake resistance. Commission of the European Communities. Doc CEN/TC250/SC8/N335,

García-Jerez A., Luzón F. \& Navarro M (2008). Determination of the elastic properties and the depth of shallow sedimentary deposits applying a spatial autocorrelation method.

Geomorphology 93: 74-88.

Herak, M. (2007). "A MatLab tool to model horizontal to vertical spectral ratio of ambient noise". Computers Geosciencies

Henstridge D.J (1979). A signal processing method for circular arrays. Geophysics 44, 179-184.

Hernández del Pozo (Análisis metodológico de la cartografía geotécnica urbana, aplicación a la ciudad de Granada) Granada, año 1998.

Kagawa T. and Group for Spanish-Japanese Joint Work on Microzonation of Granada Basin. In Joint Study on Seismic Microzonation in Granada Basin, Spain, 64-81.Tokyo Institute of Technology,

Lachet, C. and Bard, P.Y.(1994). "Numerical and theoretical investigations on the possibilitiesand limitations of Nakamura's Technique". J. Phys. Earth 42, 377-397.

Maresca, L. Nardone, Galluzzo, M. de la Rocca (2006). "Application of the Spac Method to ambient noise record in the Vesubius area (Italy). Third International Symposium on the effects of surface geology on seismic motion, Grenoble France paper 073

Microtremor Array Observation in the Granada Basin, Southern Spain (1999). 
Nakamura, Y (1989). " A method for dynamic characteristics estimation of surface using microtremor on the ground surface". Geotechnical Eng. and Disaster Prevention Laboratory, QR of RTRI, Vol 30, No 1, 25-33.

Navarro M., Corchete V., Badal J.I., Pujades L., Canas J.A. \& Vidal, F (1997). Inversion of Rg waveforms in southern Spain. Bull. Seism. Soc. Am. 87, 847-865.

Navarro, M., F.J. Sánchez, M. Fetiche, F. Vidal, T. Enomoto, T. Iwatate, I. Matsuda, T. Maeda (2002) Statical estimation for dynamic characteristics of existing buildings in Granada, Spain, using microtremors. Structural Dynamics, Eurodyn2002, Vol. 1, 807-812, Balkema.

Navarro M., García-Jerez JA., Vidal F., Enomoto T, Feriche M (2010). Vs30 structure of Granada town (southern Spain) from ambient noise array observations. 14th European Conference on Earthquake Engineering, Macedonia, 30th August-3rd September, 2010. 8 pp.

NCSE-02 (2002). “Normativa de Construcción Sismorresistente Española. Comisión Permanente de Normas Sismorresistentes", Real Decreto 997/2002. Boletín Oficial Del Estado No. 244, 11 October, 2002, Spain.

NEHRP (2003). Recommendation prevision for seismic regulation for new buildings and other structures, Building Seismic Saftey Council, Washington, D. C Federal Emergency. FEMA.

Parolai S., Picozzi M., Richwalski S.M. \& Milkereit C (2005). Joint inversion of phase dispersion and $\mathrm{H} / \mathrm{V}$ ratio curves from seismic noise recordings using a genetic algorithm. Geophysical Research Letters, Vol. 32, L01303.

Rodriguez Salas. Curso Aplicado de Cimentaciones.

Seo, K. (1999). "Joint Study on Seismic Microzonation in Granada Basin, Spain", Report of research project, Grant.In-Aid for international scientific research, 1995-1997, Project № 07044136, Tokyo Institute of Technology.

Tokimatsu K (1997). Geotechnical site characterization using surface waves. In: Ishihara (Ed.), Earthquake Geotechnical Engineering. Balkema, Rótterdam, pp. 1333-1368.

Trigo Salas, T.A. (2007). " Influencia de la amplificación local de las ondas sísmicas y de la interacción suelo-fundación en el puente Marga-Marga" Universidad de Chile

Tsai, N. (1969). " Influence of local geology on earthquake ground motion" . California Institute of Technology, Pasadena California.

Vidal F (1986). Sismotectónica de la región Béticas-Mar de Alborán. Tesis Doctoral. Universidad de Granada. 
Apéndice 1. Medidas de ruido obtenidas en los diferentes emplazamientos Registros de microtremores que se obtuvieron en los diferentes arrays.
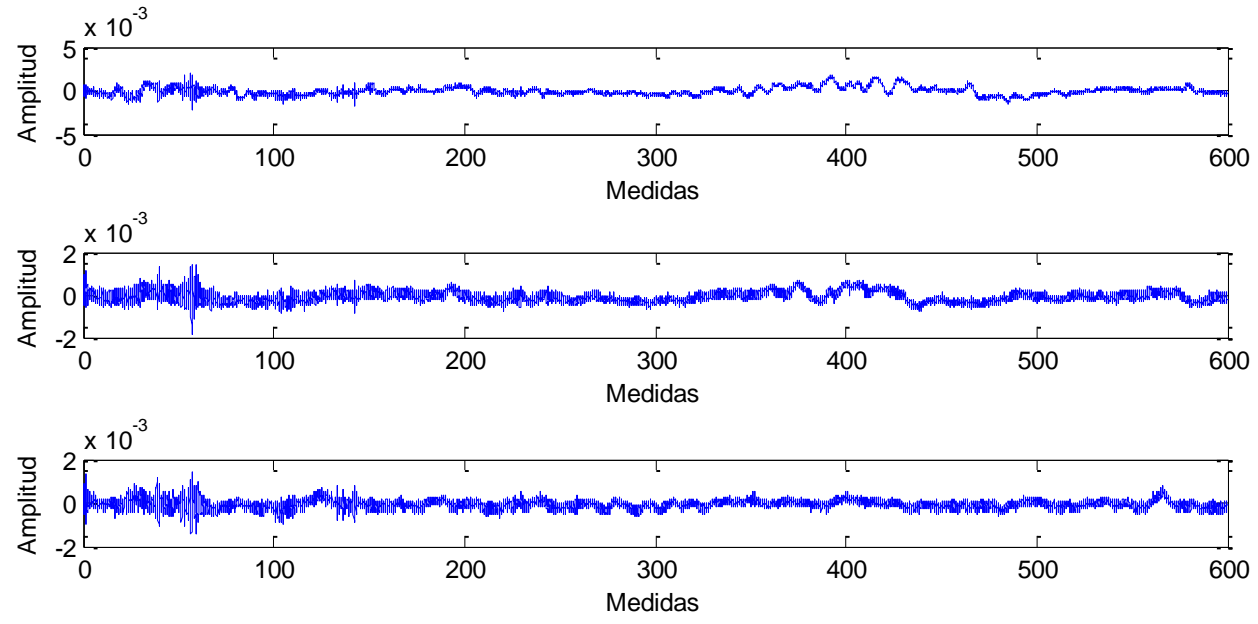

Figura A.1. Ejemplo medidas de microtremores en el array de Ainadamar.
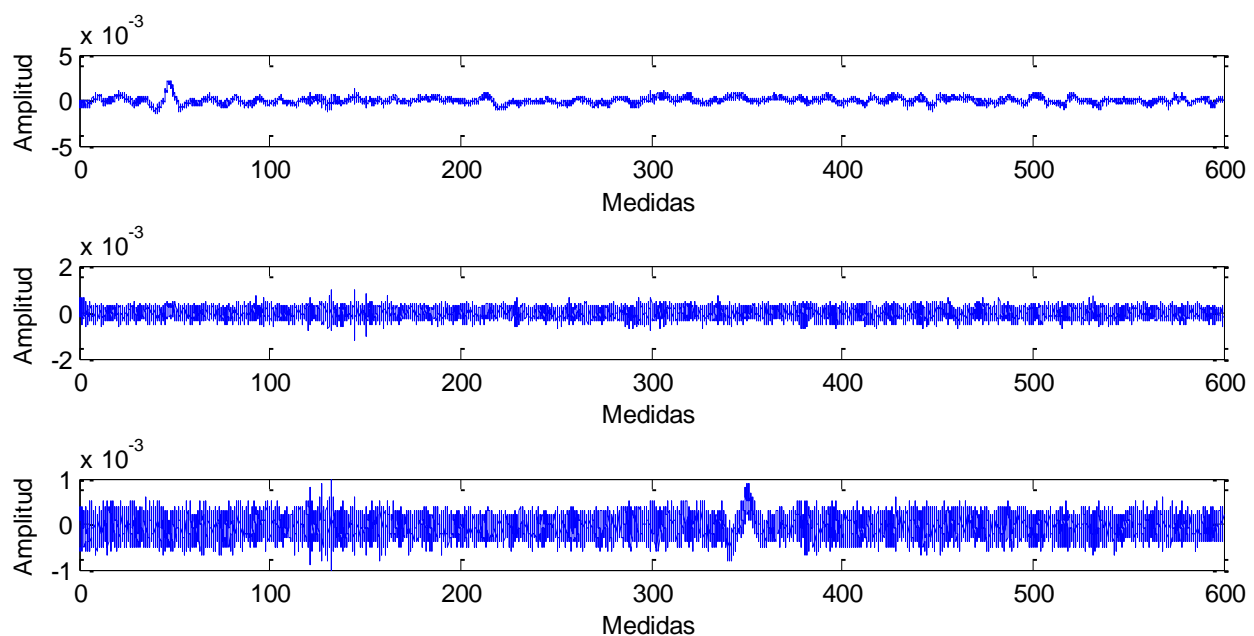

Figura A.2. Ejemplo medidas de microtremores en el array de Beiro. 

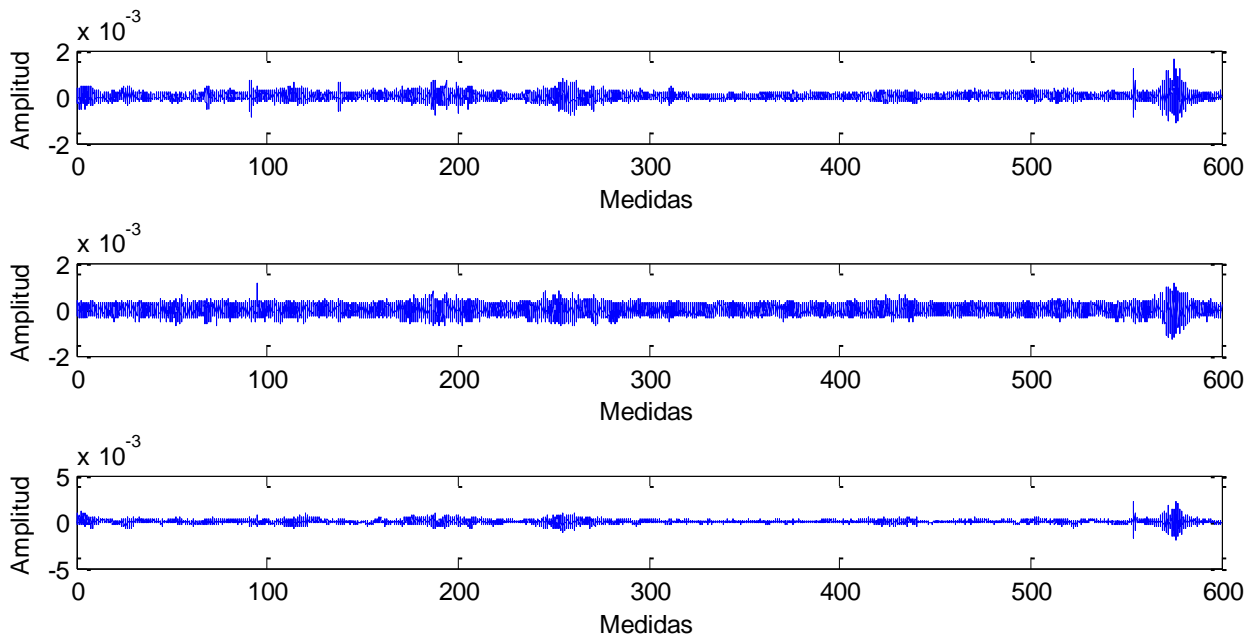

Figura A.3. Ejemplo medidas de microtremores en el array de Bola de oro.
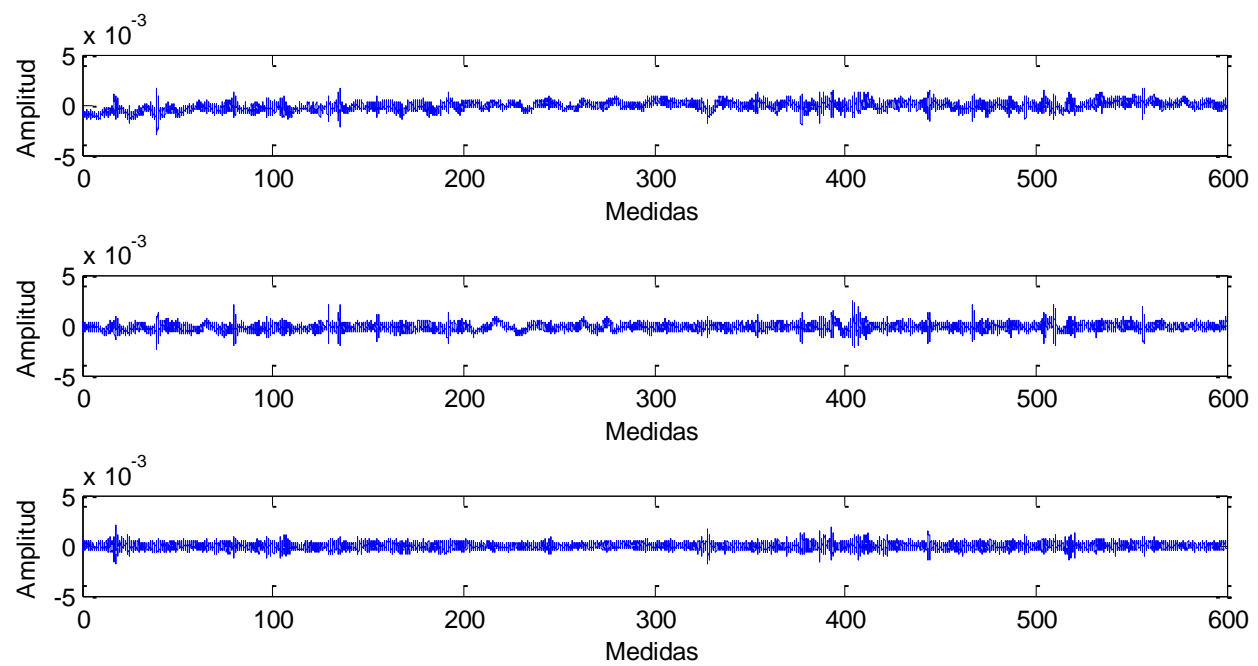

Figura A.4. Ejemplo medidas de microtremores en el array de Chana. 

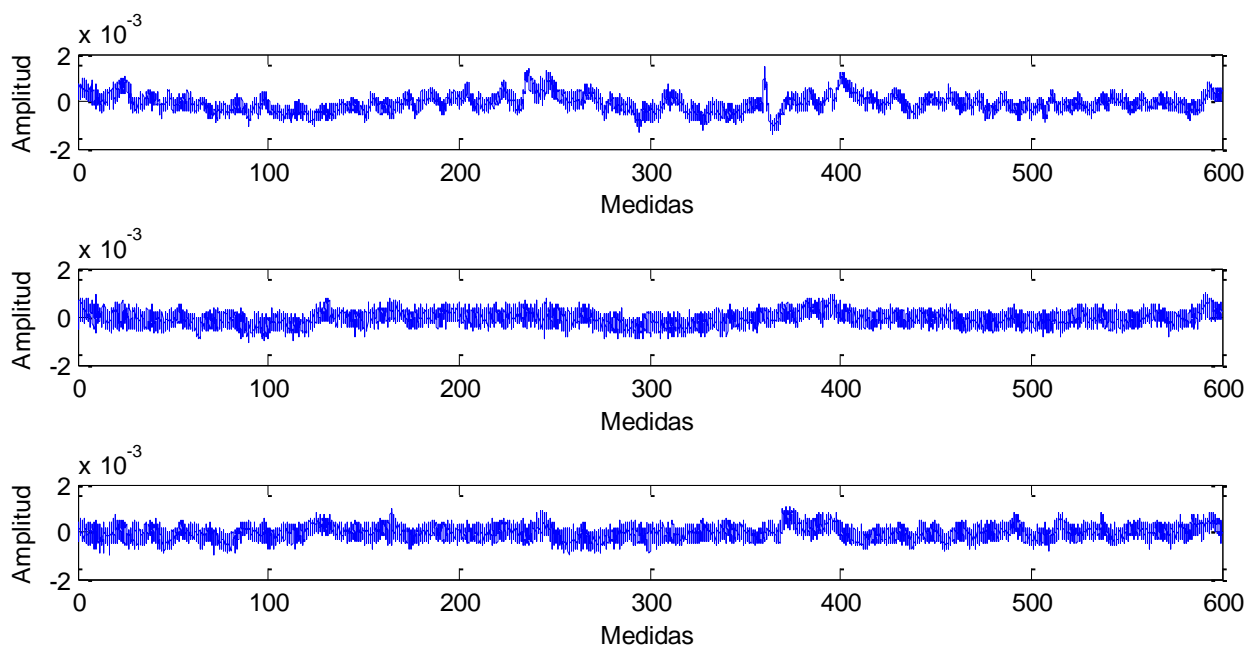

Figura A.5. Ejemplo medidas de microtremores en el array de Fuentenueva.
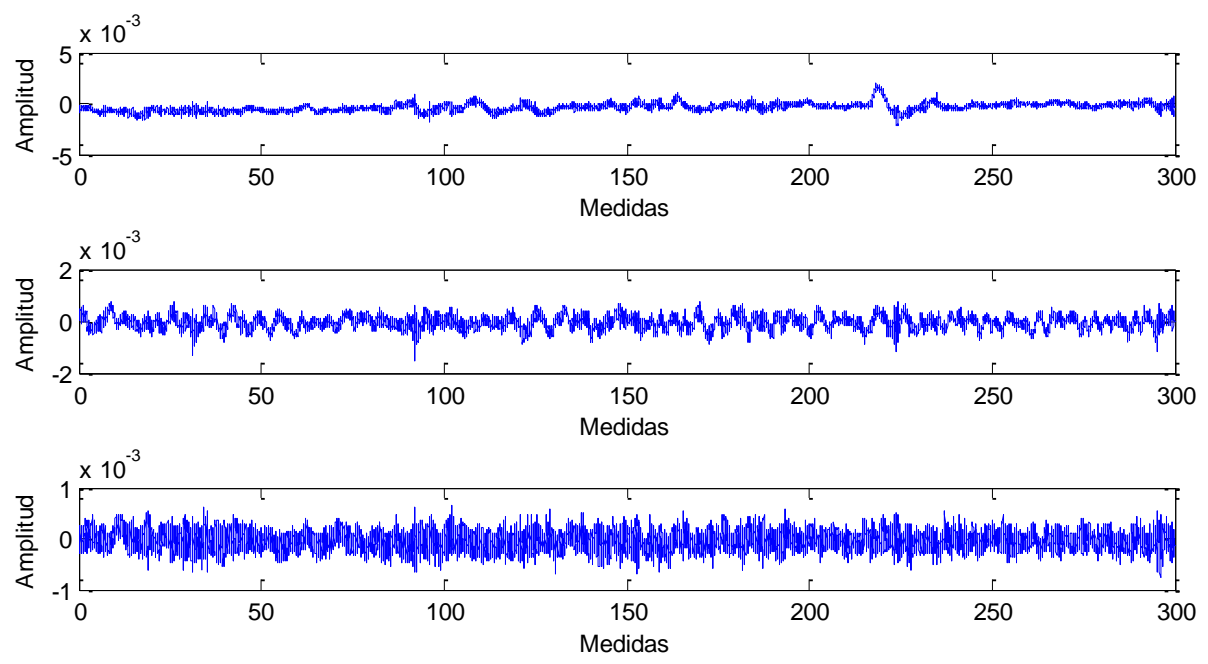

Figura A.6. Ejemplo medidas de microtremores en el array de Granada 74. 

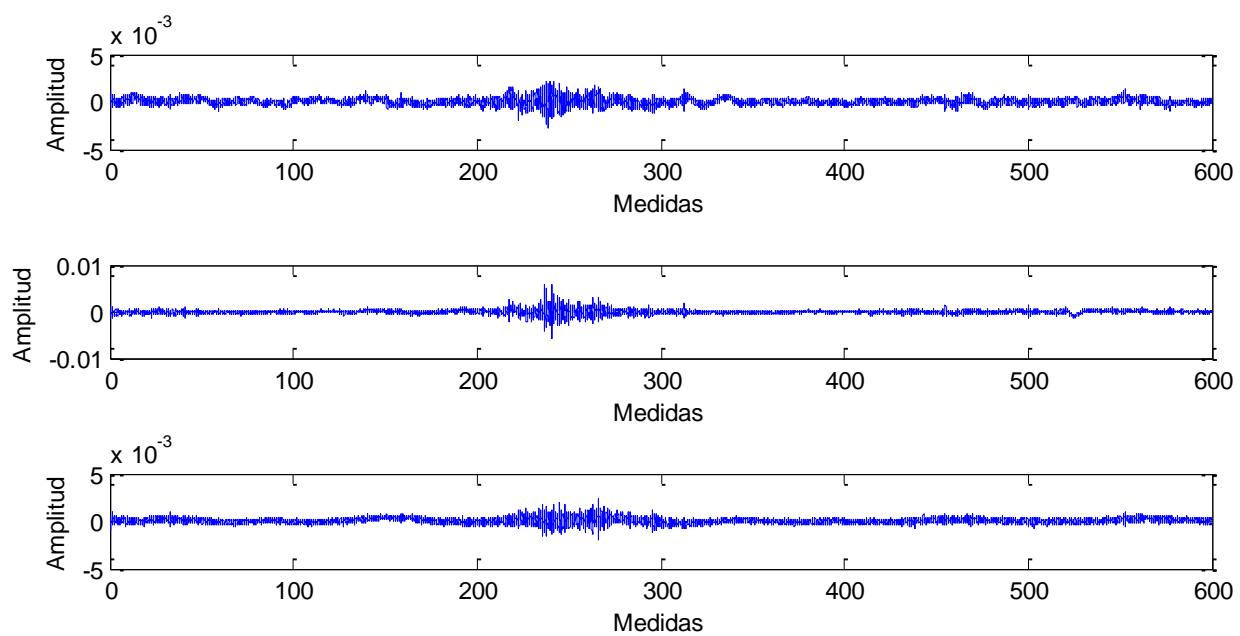

Figura A.7. Ejemplo medidas de microtremores en el array de Estadio de la Juventud
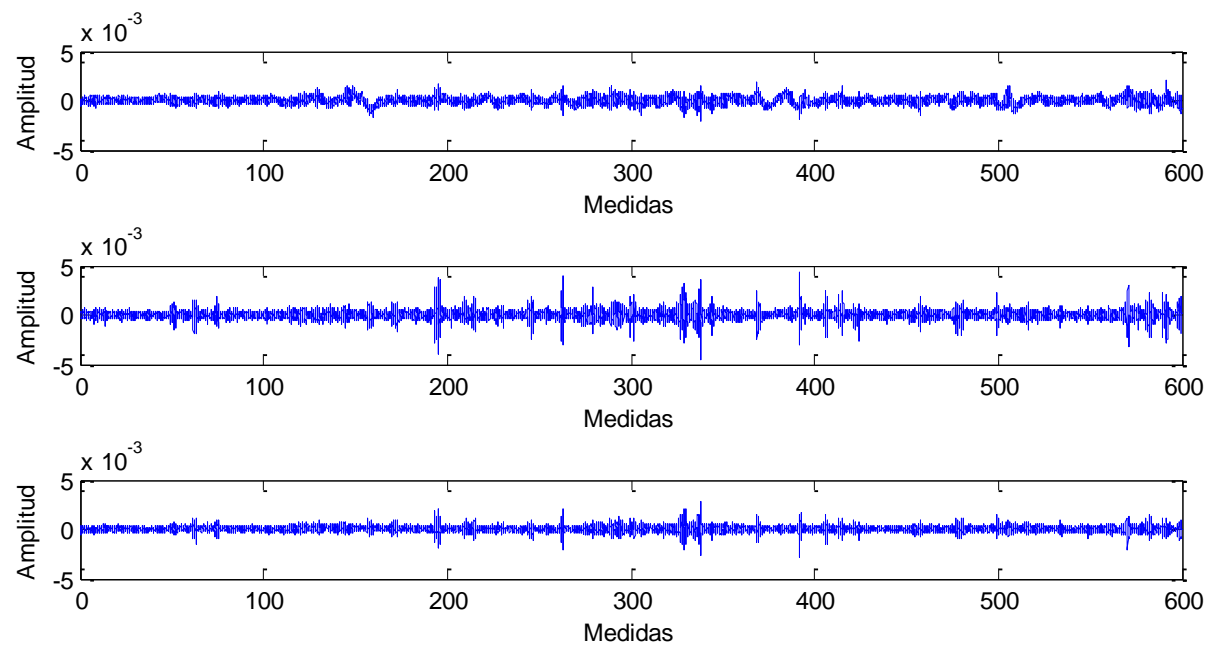

FiguraA.8. Ejemplo medidas de microtremores en el array de Náyades 

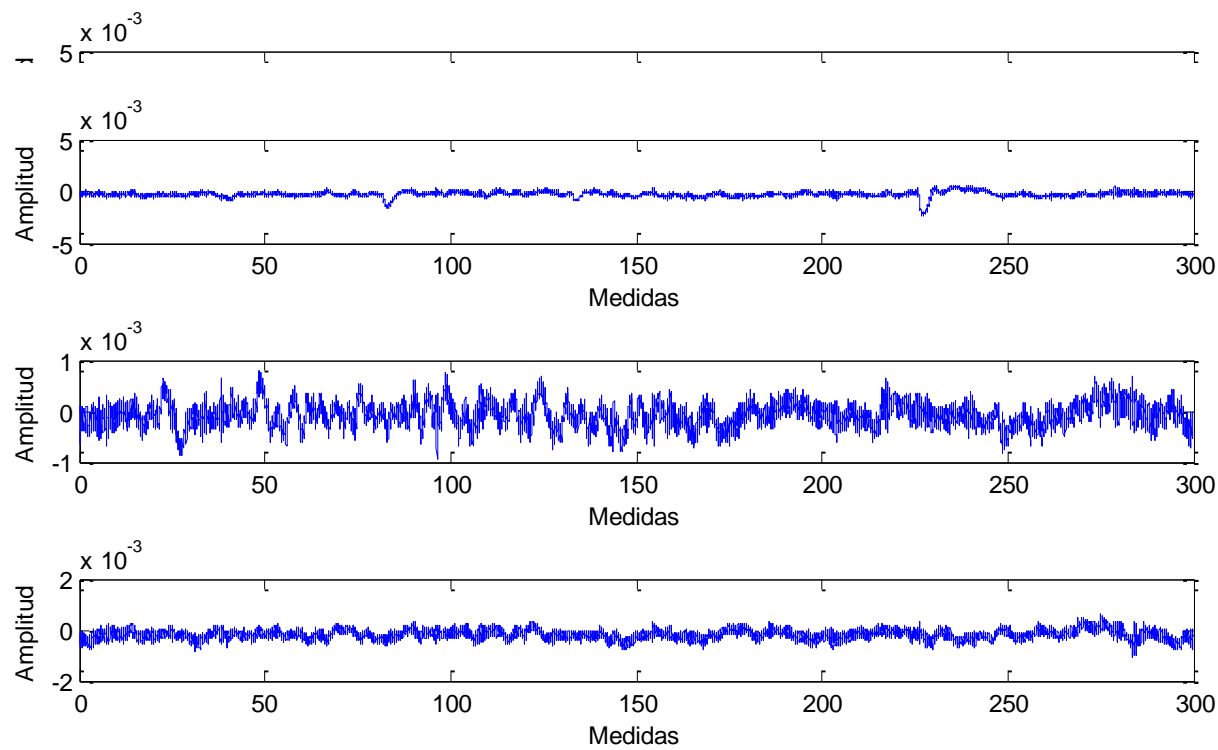

Figura A.9 Ejemplo medidas de microtremores en el array de Parque de las Ciencias
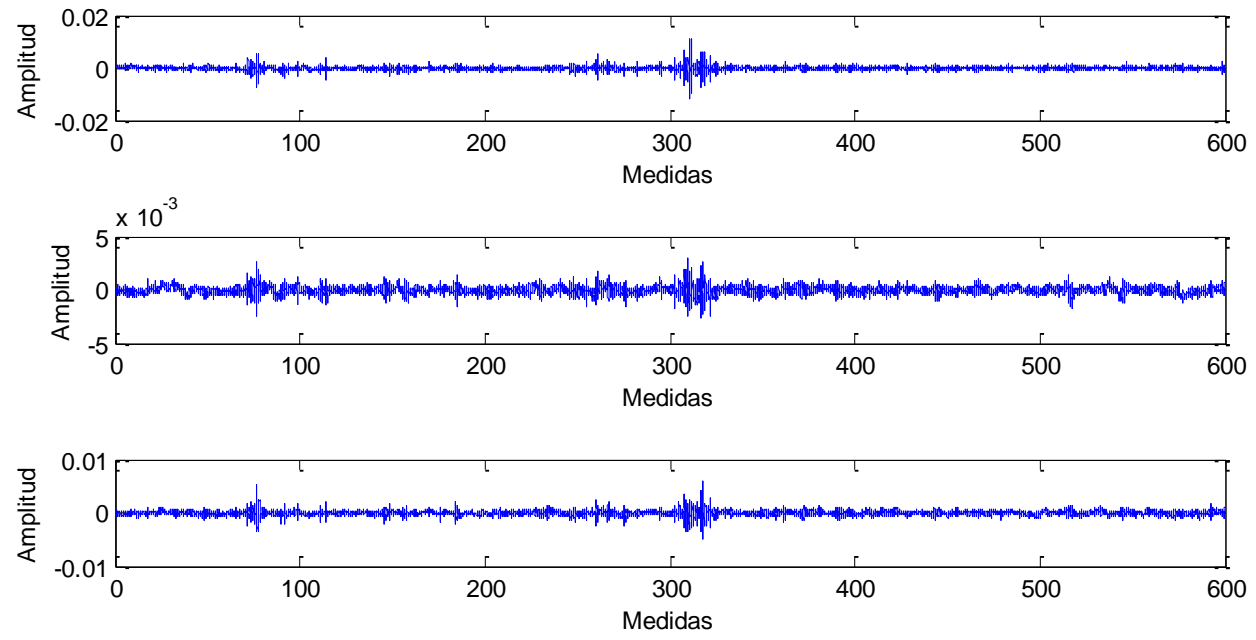

Figura A.10. Ejemplo medidas de microtremores en el array de Sanders 

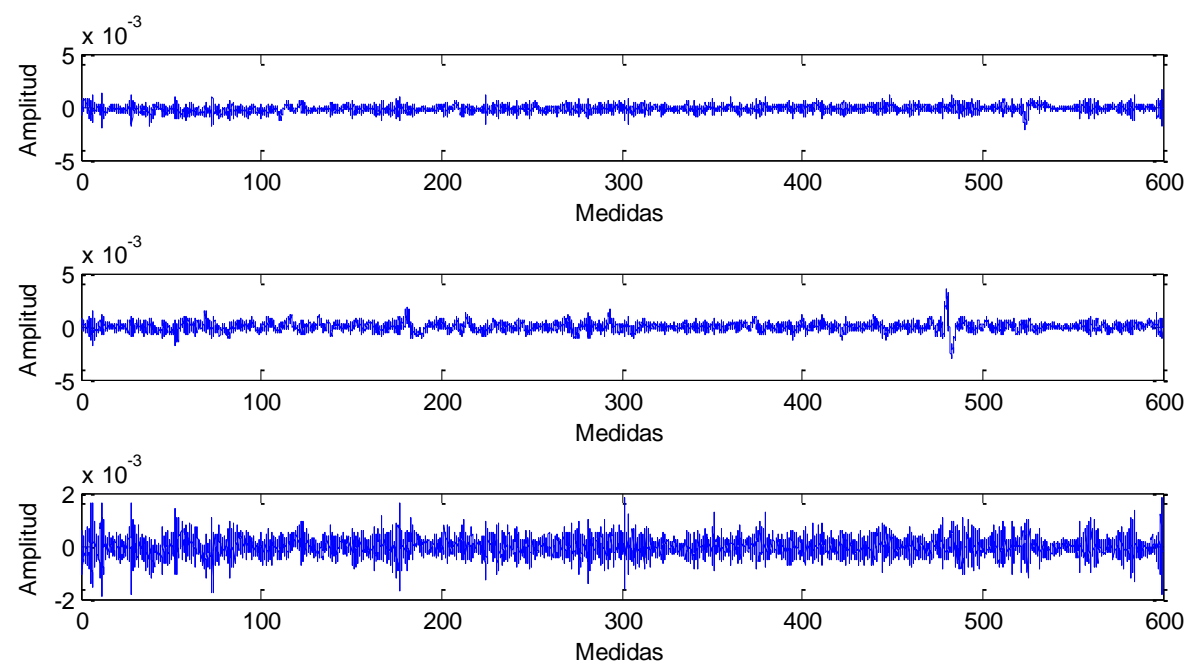

Figura A.11. Ejemplo medidas de microtremores en el array de Vega
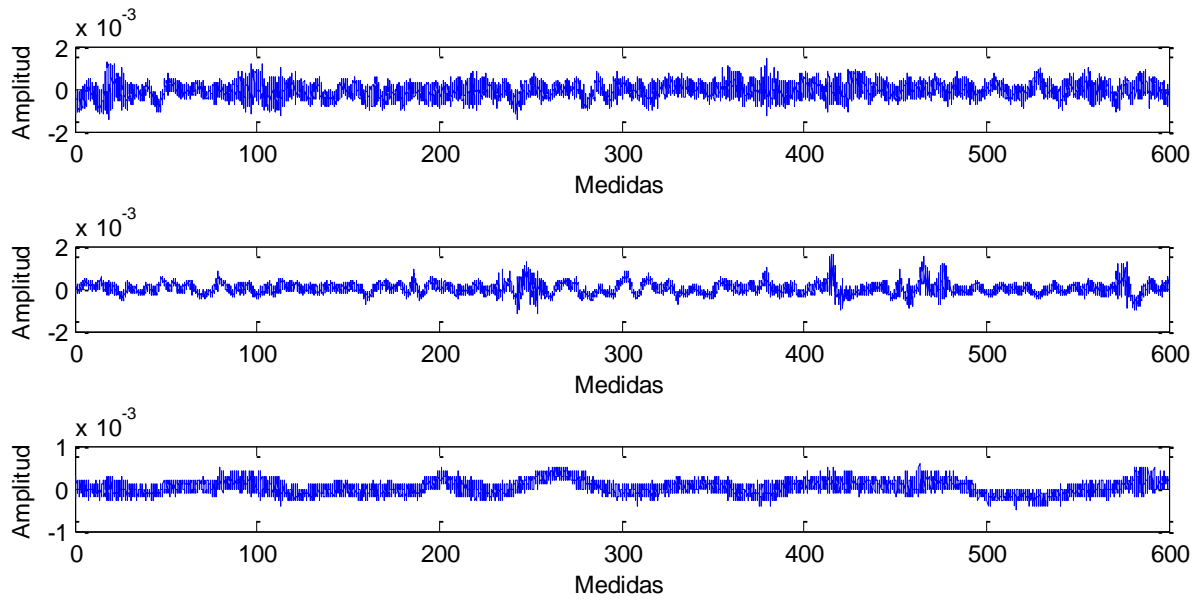

Figura A.12. Ejemplo medidas de microtremores en el array de Zaidín 


\section{Apéndice 2. Características de los registros usados}

\section{Características de los registros del terremoto de Valnerina}

\begin{tabular}{|c|c|c|c|c|c|c|c|c|}
\hline \multicolumn{4}{|c|}{ ECODE SCODE WCODE FileName } & \multicolumn{2}{|c|}{ Earthquake } & \multicolumn{3}{|c|}{ Channel Format } \\
\hline 115 & 225 & 242 & 000242XA.COR & Valnerina & Cascia & $\mathrm{N}-\mathrm{S}$ & \multicolumn{2}{|c|}{ Native ASCII with header } \\
\hline 115 & 225 & 242 & 000242YA.COR & Valnerina & Cascia & $E-W$ & \multicolumn{2}{|c|}{ Native ASCII with header } \\
\hline 115 & 225 & 242 & 000242ZA.COR & Valnerina & Cascia & VERT & \multicolumn{2}{|c|}{ Native ASCII with header } \\
\hline 115 & 61 & 246 & 000246XA.COR & Valnerina & \multicolumn{2}{|c|}{ Arquata del Tronto } & N-S & Native ASCII with header \\
\hline 115 & 61 & 246 & 000246YA.COR & Valnerina & \multicolumn{2}{|c|}{ Arquata del Tronto } & $E-W$ & Native ASCII with header \\
\hline 115 & 61 & 246 & 000246ZA.COR & Valnerina & \multicolumn{2}{|c|}{ Arquata del Tronto } & VERT & Native ASCII with header \\
\hline 115 & 279 & 247 & 000247XA.COR & Valnerina & Spoleto & $\mathrm{N}-\mathrm{S}$ & \multicolumn{2}{|c|}{ Native ASCII with header } \\
\hline 115 & 279 & 247 & 000247YA.COR & Valnerina & Spoleto & $E-W$ & \multicolumn{2}{|c|}{ Native ASCII with header } \\
\hline 115 & 279 & 247 & 000247ZA.COR & Valnerina & Spoleto & VERT & \multicolumn{2}{|r|}{ Native ASCII with header } \\
\hline
\end{tabular}

\section{Características de los registros del terremoto del "Basso Tirreno"}

\begin{tabular}{|c|c|c|c|c|c|c|c|c|}
\hline \multicolumn{4}{|c|}{ ECODE SCODE WCODE FileName } & \multicolumn{2}{|l|}{ Earthquake } & \multicolumn{3}{|c|}{ Channel Format } \\
\hline 81 & 47 & 171 & 000171XA.COR & Basso Tirreno & Naso & NS & \multicolumn{2}{|c|}{ Native ASCII with header } \\
\hline 81 & 47 & 171 & 000171YA.COR & Basso Tirreno & Naso & EW & \multicolumn{2}{|c|}{ Native ASCII with header } \\
\hline 81 & 47 & 171 & 000171ZA.COR & Basso Tirreno & Naso & VERT & \multicolumn{2}{|c|}{ Native ASCII with header } \\
\hline 81 & 46 & 170 & 000170XA.COR & Basso Tirreno & \multicolumn{3}{|c|}{ Patti-Cabina Prima NS } & Native ASCII with header \\
\hline 81 & 46 & 170 & 000170YA.COR & Basso Tirreno & \multicolumn{3}{|c|}{ Patti-Cabina Prima EW } & Native ASCII with header \\
\hline 31 & 46 & 170 & 000170ZA.COR & Basso Tirreno & Patti-Cabina & Prima & VERT & Native ASCII with header. \\
\hline
\end{tabular}

